\title{
Optimal sampling recovery of mixed order Sobolev embeddings via discrete Littlewood-Paley type characterizations
}

\author{
Glenn Byrenheid ${ }^{a *}$ Tino Ullrich $^{a}$ \\ ${ }^{a}$ Hausdorff-Center for Mathematics/Institute for Numerical Simulation \\ Endenicher Allee 62, 53115 Bonn, Germany
}

September 18, 2018

\begin{abstract}
In this paper we consider the $L_{q}$-approximation of multivariate periodic functions $f$ with $L_{p}$-bounded mixed derivative (difference). The (possibly non-linear) reconstruction algorithm is supposed to recover the function from function values, sampled on a discrete set of $n$ sampling nodes. The general performance is measured in terms of (non-)linear sampling widths $\varrho_{n}$. We conduct a systematic analysis of Smolyak type interpolation algorithms in the framework of Besov-Lizorkin-Triebel spaces of dominating mixed smoothness based on specifically tailored discrete Littlewood-Paley type characterizations. As a consequence, we provide sharp upper bounds for the asymptotic order of the (non-)linear sampling widths in various situations and close some gaps in the existing literature. For example, in case $2 \leq p<q<\infty$ and $r>1 / p$ the linear sampling widths $\varrho_{n}^{\operatorname{lin}}\left(S_{p}^{r} W\left(\mathbb{T}^{d}\right), L_{q}\left(\mathbb{T}^{d}\right)\right)$ and $\varrho_{n}^{\operatorname{lin}}\left(S_{p, \infty}^{r} B\left(\mathbb{T}^{d}\right), L_{q}\left(\mathbb{T}^{d}\right)\right)$ show the asymptotic behavior of the corresponding Gelfand $n$-widths, whereas in case $1<p<q \leq 2$ and $r>1 / p$ the linear sampling widths match the corresponding linear widths. In the mentioned cases linear Smolyak interpolation based on univariate classical trigonometric interpolation turns out to be optimal.
\end{abstract}

MSC 2010. 42A10, 42A15, 41A46, 41A58, 41A63, 41A17, 41A25, 42B25, 42B35

Keywords. sampling recovery, sparse grids, sampling representations, Besov-Triebel-Lizorkin spaces of mixed smoothness, Smolyak algorithm, Gelfand $n$-widths, linear widths

\section{Introduction}

This paper is concerned with the problem of optimal sampling recovery in classes of multivariate functions. We consider the approximation of $d$-variate functions $f$ from classes with $L_{p}$-bounded mixed derivative (difference) in $L_{q}$. We aim for the exact asymptotic order of the sampling widths which measure the minimal worst-case error for the (linear) sampling recovery problem with $n$ points. To be more precise, we measure the performance of an optimal sampling algorithm with the linear sampling widths

$$
\varrho_{n}^{\operatorname{lin}}(\boldsymbol{F}, Y):=\inf _{X_{n}} \inf _{\Psi_{n}} \sup _{\|f \mid \boldsymbol{F}\| \leq 1}\left\|f-\sum_{i=1}^{n} f\left(\boldsymbol{x}^{i}\right) \psi_{i}(\cdot)\right\|_{Y} \quad, \quad n \in \mathbb{N},
$$

${ }^{*}$ Corresponding author. Email: byrenheid.glenn@gmail.com 
where the sampling nodes $X_{n}:=\left\{\boldsymbol{x}^{i}\right\}_{i=1}^{n} \subset \mathbb{T}^{d}$ and associated (continuous) functions $\Psi_{n}:=$ $\left\{\psi_{i}\right\}_{i=1}^{n}$ determine a linear sampling recovery algorithm which is fixed in advance for a class $\mathbf{F}$ of multivariate functions on the $d$-torus $\mathbb{T}^{d}$. Here the error is measured in $Y=L_{q}$. Let us emphasize that in (1.1) we restrict to linear recovery algorithms, whereas we admit general recovery algorithms $\varphi: \mathbb{C}^{n} \rightarrow L_{q}$ in the definition of the (non-linear) sampling widths

$$
\varrho_{n}(\mathbf{F}, Y):=\inf _{\varphi, X_{n}} \sup _{\|f \mid \mathbf{F}\| \leq 1}\left\|f-\varphi\left(X_{n}(f)\right)\right\|_{Y},
$$

which is also denoted as the worst-case error for standard information, see [26, Sect. 4.1]. We are particularly interested in optimal point sets $X_{n}$ and corresponding recovery algorithms which yield the correct asymptotic order of (1.1) and (1.2).

The interest in this topic goes back to 1963 and started with Smolyak [38] who considered uniform approximation of multivariate functions with mixed smoothness on the basis of function values. He used an influential construction which is nowadays known as Smolyak's algorithm

$$
T_{m} f:=\sum_{\substack{\boldsymbol{j} \in \mathbb{N}_{0}^{d} \\|\boldsymbol{j}|_{1} \leq m}}\left(L_{j_{1}}-L_{j_{1}-1}\right) \otimes \ldots \otimes\left(L_{j_{d}}-L_{j_{d}-1}\right) f \quad, \quad m \in \mathbb{N},
$$

where the $\left(L_{j}\right)_{j \in \mathbb{N}_{0}}$ represent univariate approximation operators (put $L_{-1}:=0$ ). For more historical comments see [12, Sect. 5]. When applied to a univariate sampling (interpolation) scheme $\left(I_{j}\right)_{j}$ this construction yields a powerful sampling (interpolation) algorithm for the multivariate case taking points from a so-called sparse grid.

\subsection{New matching bounds for (non-)linear sampling recovery}

In this paper we investigate the optimal sampling recovery problem for the embedding

$$
\text { Id }: S_{p, \theta}^{r} F\left(\mathbb{T}^{d}\right) \rightarrow L_{q}\left(\mathbb{T}^{d}\right)
$$

where $0<p<q \leq \infty, 0<\theta \leq \infty$ and $\boldsymbol{r}>1 / p$. Without loss of generality we assume

$$
r=r_{1}=\ldots=r_{\mu}<r_{\mu+1} \leq \ldots \leq r_{d}<\infty \quad, \quad \mu \leq d
$$

The main goal of this paper is to present a systematic framework towards new upper bounds for sampling recovery on Smolyak grids in Sobolev-Triebel-Lizorkin spaces $S_{p, \theta}^{r} F\left(\mathbb{T}^{d}\right)$ with dominating mixed smoothness. One of the main results in this paper is the sharp rate of convergence

$$
\varrho_{n}^{\operatorname{lin}}\left(S_{p, \theta}^{r} F\left(\mathbb{T}^{d}\right), L_{q}\left(\mathbb{T}^{d}\right)\right) \asymp\left(\frac{(\log n)^{\mu-1}}{n}\right)^{r-1 / p+1 / q} \quad, \quad n \in \mathbb{N},
$$

whenever $1<p<q \leq 2,1 \leq \theta \leq \infty$ or $2 \leq p<q<\infty, 2 \leq \theta \leq \infty$ and $r>1 / p$, see Corollary 7.2 below. Our main contribution is the constructive upper bound which holds true whenever $0<p<q<\infty, 0<\theta \leq \infty$ and $r>1 / p$. This is complemented by (see Theorem 6.4)

$$
\varrho_{n}^{\operatorname{lin}}\left(S_{p, \theta}^{r} F\left(\mathbb{T}^{d}\right), L_{\infty}\left(\mathbb{T}^{d}\right)\right) \lesssim\left(\frac{(\log n)^{\mu-1}}{n}\right)^{r-1 / p}(\log n)^{(\mu-1)(1-1 / p)_{+}} \quad, \quad n \in \mathbb{N} .
$$

The upper bounds are realized by an explicit family of interpolation operators $T_{m}^{L}$ using $n \asymp 2^{m} m^{\mu-1}$ function values on a (anisotropic) Smolyak grid, where the parameter $L \in \mathbb{N}$ refers to the polynomial decay of the univariate fundamental interpolant $(L=1$ Dirichlet 
kernel, $L=2$ de la Vallée Poussin type kernels, $L>2$ higher order kernels). It turned out that, for the sampling recovery problem (1.4) and the upper bounds in (1.6), (1.7), (1.8), (1.9), the condition $L>1 / q$ is sufficient, which means that Smolyak's algorithm (1.3) applied to the classical trigonometric interpolation (based on the Dirichlet kernel (1.12) does the job. For $\theta=p=2$ in (1.7) this has been already observed in [2, Rem. 6.12].

Let us emphasize the important special case $(\theta=2)$, where it holds the identification $S_{p, \theta}^{r} F\left(\mathbb{T}^{d}\right)=S_{p}^{r} W\left(\mathbb{T}^{d}\right)$ with the space of functions with bounded mixed derivative. As a corollary from (1.6) we obtain the new sharp rate of convergence

$$
\varrho_{n}^{\operatorname{lin}}\left(S_{p}^{r} W\left(\mathbb{T}^{d}\right), L_{q}\left(\mathbb{T}^{d}\right)\right) \asymp\left(\frac{(\log n)^{\mu-1}}{n}\right)^{r-1 / p+1 / q} \quad, \quad n \in \mathbb{N},
$$

in case $1<p<q \leq 2$ or $2 \leq p<q<\infty$ and $\boldsymbol{r}>1 / p$ which was unknown before. The upper bound is achieved with sparse grid interpolation based on classical univariate trigonometric interpolation. In particular, this improves on the bounds stated by Triebel in [4, Thm. 4.15, Cor. 4.16] in case $r=1$. The parameter domain where (1.8) holds is shown in the left diagram, where the parameters $\alpha$ and $\beta$ refer to the following rate of convergence

$$
\varrho_{n}\left(\mathbf{F}, L_{q}\right) \asymp\left(\frac{(\log n)^{\mu-1}}{n}\right)^{\alpha}(\log n)^{(\mu-1) \beta} .
$$

The precise statements can be found in Sections 7,8 ,
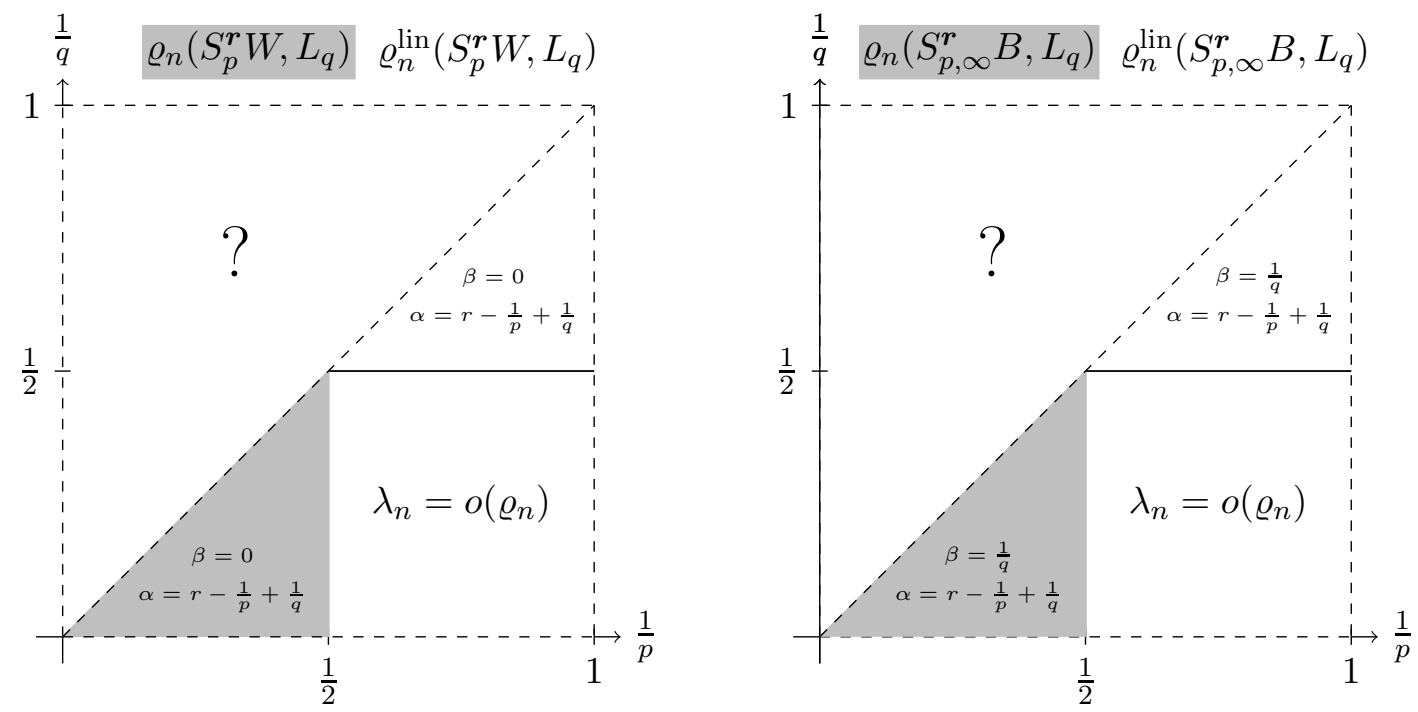

Figure 1: Linear and non-linear sampling widths.

We mainly contributed to the upper bounds in the left figure. Most of the results illustrated in the right figure for Hölder-Nikolskij spaces $S_{p, \infty}^{r} B\left(\mathbb{T}^{d}\right)$ of mixed smoothness are well-known. Note that Open Problem 5.3 in [12] refers to the lower triangle in the right Figure 1] A new approach of Malykhin and Ryutin [23] settled this question for linear sampling recovery, cf. Corollary 7.6. We observed that their method also bounds the Gelfand widths from below, cf. Theorem 8.3 and Corollary 8.4. This yields the optimal order also for the non-linear sampling widths (1.2), which is illustrated by the shaded lower triangles in Figure 1, The matching bound in the right upper triangle for $S_{p, \infty}^{r} B\left(\mathbb{T}^{d}\right)$ were obtained by Dinh Dũng [5, 6]. The 
necessary benchmark results on linear widths were obtained by Galeev [15, 16], Romanyuk [29, 30], and the recent paper by Malykhin and Ryutin [23]. Note, that all sharp upper bounds can be realized by Smolyak type operators (1.3), i.e. via linear interpolation on sparse grids based on univariate Dirichlet interpolation, (1.11), (1.12). What concerns Besov spaces with bounded mixed difference $S_{p, \theta}^{r} B\left(\mathbb{T}^{d}\right)$ it is known that

$$
\varrho_{n}^{\operatorname{lin}}\left(S_{p, \theta}^{r} B\left(\mathbb{T}^{d}\right), L_{q}\left(\mathbb{T}^{d}\right)\right) \asymp\left(\frac{(\log n)^{\mu-1}}{n}\right)^{r-1 / p+1 / q}(\log n)^{(\mu-1)(1 / q-1 / \theta)_{+}} \quad, \quad n \in \mathbb{N},
$$

if $1<p<q \leq 2,1 \leq \theta<\infty$ and $\boldsymbol{r}>1 / p$, see [12, Thm. 4.47, 5.15] and the references therein. With our method we can show the upper bound in case $0<p<q \leq \infty, 0<\theta \leq \infty$ and $r>1 / p$, see Theorem 6.6, with interpolation operators providing $L>1 / q$. Comparing to (1.6) there is an extra log-term in (1.9) in case of "large" $\theta>q$. There are still many open cases in this framework which actually lack the suitable lower bounds.

Let us refer to the works by Temlyakov [41, 42] and the more recent papers Sickel, Ullrich [35, 36, 46], Dinh Dũng [10, 11], [2], as well as [12] and the references therein for upper bounds in case $p \geq q$ and the question-marked region. We emphasize that our technique allows to reproduce all those results, including the upper bound in [41, within a few lines of proof.

In Open Problem 18 in [26, Sect. 4.2.4] the authors conjecture the equivalence $\varrho_{n}^{\operatorname{lin}} \asymp \varrho_{n}$ for all parameters $1<p, q<\infty$ in case of isotropic Sobolev spaces $W_{p}^{r}(\Omega)$ on bounded Lipschitz domains $\Omega$, see also Novak, Triebel [25] and Heinrich [18, Thms. 5.2, 5.3]. In the present paper we consider mixed smoothness periodic Sobolev embeddings. In our case, the conjecture is true if $2 \leq p<q<\infty$ for both Sobolev and Hölder-Nikolskij spaces, see the shaded regions in the diagrams above. In all other cases it is not known. Our results also support the above conjecture in the mixed smoothness setting. A similar statement as in [26, Rem. 4.18], namely the equivalence $\lambda_{n} \asymp \varrho_{n}^{\text {lin }}$ if $p<q$ are on the same side of 2 and $\lambda_{n}=o\left(\varrho_{n}^{\operatorname{lin}}\right)$ if $p<2<q$ is also true in our case.

\subsection{Discrete Littlewood-Paley type characterizations}

In Definition 3.3 below we introduce Besov-Lizorkin-Triebel spaces of mixed smoothness via Fourier analytic building blocks $\delta_{\boldsymbol{j}}[f]$ generated by a dyadic decomposition of unity. In this paper we aim for function space characterizations where we replace the building blocks $\delta_{j}[f]$ by the blocks

$$
q_{j}[f]=\left(I_{j_{1}}-I_{j_{1}-1}\right) \otimes \ldots \otimes\left(I_{j_{d}}-I_{j_{d}-1}\right) f \quad, \quad j \in \mathbb{N}_{0}^{d},
$$

used in the classical Smolyak algorithm (see (1.3) above). Here the operators $\left(I_{j}\right)_{j}$ are univariate interpolation operators

$$
I_{j}^{L}[f]=\sum_{u=0}^{2^{j}-1} f\left(\frac{2 \pi u}{2^{j}}\right) K_{\pi, j}^{L}\left(\cdot-\frac{2 \pi u}{2^{j}}\right) .
$$

In a way we replace the usual convolution by a discrete one such that the building blocks $q_{j}^{L}[f]$ are constructed out of $\asymp 2^{|\boldsymbol{j}|_{1}}$ function values. The parameter $L \in \mathbb{N}$ refers to the decay of the fundamental interpolant $K_{\pi, j}^{L}$, which represents a suitable trigonometric polynomial of degree $2^{j}$ and will be explicitly constructed in Section 2. In case $L=1$ we have the classical univariate nested trigonometric interpolation, where $K_{\pi, j}^{1}:=2^{-j} \mathcal{D}_{j}^{1}$ with $\mathcal{D}_{0}^{1} \equiv 1$ and

$$
\mathcal{D}_{j}^{1}(x):=\mathcal{D}_{2^{j-1}}(x)-e^{i 2^{j-1} x}=e^{-i\left(2^{j-1}-1\right) x} \frac{e^{i 2^{j} x}-1}{e^{i x}-1} \quad, \quad x \in \mathbb{T},
$$


for $j \in \mathbb{N}$. The parameter $L=2$ refers to de la Vallée Poussin type operators and $L>2$ to higher order kernels.

We will prove the following characterization for Sobolev spaces of mixed smoothness if $\boldsymbol{r}>\max \{1 / p, 1 / 2\}$ and $1<p<\infty$

$$
\left\|f \mid S_{p}^{r} W\left(\mathbb{T}^{d}\right)\right\| \asymp\left\|\left(\sum_{\boldsymbol{j} \in \mathbb{N}_{0}^{d}} 2^{2 \boldsymbol{j} \cdot \boldsymbol{r}}\left|q_{\boldsymbol{j}}^{L}[f](\cdot)\right|^{2}\right)^{1 / 2}\right\|_{p},
$$

where we may use $L \geq 1$, i.e. Dirichlet type characterizations are admitted. This result provides a powerful tool to deal with Sobolev embeddings $S_{p}^{r} W\left(\mathbb{T}^{d}\right)$ in $L_{q}\left(\mathbb{T}^{d}\right)$. Analyzing Smolyak's algorithm (1.3) in this context has been a technical issue in the past. With (1.13) and its counterpart for Triebel-Lizorkin spaces (1.14) it becomes a straight-forward computation.

For Triebel-Lizorkin spaces we obtain the representation (see Theorem 5.1)

$$
\left\|f \mid S_{p, \theta}^{r} F\left(\mathbb{T}^{d}\right)\right\| \asymp\left\|\left(\sum_{\boldsymbol{j} \in \mathbb{N}_{0}^{d}} 2^{r \cdot j \theta}\left|q_{j}^{L}[f](\cdot)\right|^{\theta}\right)^{1 / \theta}\right\|_{p}
$$

in case $0<p<\infty, 0<\theta \leq \infty, \boldsymbol{r}>\max \{1 / p, 1 / \theta\}$ and $L>\max \{1 / p, 1 / \theta\}$ (except in the case $\theta=\infty$ where $L \geq 2$ ). Note, that we encounter the well-known (and infamous) condition $\boldsymbol{r}, L>\max \{1 / p, 1 / \theta\}$ (see also (1.13) for $\theta=2$ ), which is relevant if $p>\theta$. However, this condition is most likely optimal for the respective sampling characterization. Note, that when replacing the classical smooth dyadic decomposition of unity (see Def. 3.2) in the definition of the spaces (see Def. 3.3) by a non-smooth variant like de la Vallée Poussin means, we would encounter the same condition on $L$, which may not be improved as the recent findings in [4, 33, 34 indicate. In addition, Note, that in case of quasi-Banach spaces, where $\min \{p, \theta\}<1$, we need to use sampling kernels (1.11) of higher order $L$ as the condition $L>\max \{1 / p, 1 / \theta\}$ indicates. Surprisingly, the de la Vallée Poussin type kernels work well for the characterization (1.14) if $1 / 2<p, \theta<\infty$.

Interestingly, the bounds (1.6) as well as (1.8) are valid for all $r>1 / p$, in particular for the case of "small smoothness", i.e., $1 / p<r \leq 1 / \theta$ if $p>\theta$. Note, that in this range the characterization (1.13) does not help. That is why we develop a complete theory of sampling representations also for more general Triebel-Lizorkin spaces in this paper, which allows to analyze the larger space $S_{p, \theta^{*}}^{r} F\left(\mathbb{T}^{d}\right)$ instead with $\theta^{*}>p$, which works since $\theta$ plays no role in (1.6). Approximation operators realizing the upper bounds in (1.6), (1.8) and (1.9) only depend on the integrability $q$ in the target space $L_{q}\left(\mathbb{T}^{d}\right)$, that is $L>1 / q$ will be sufficient. Hence, we observe a certain universality property of the Smolyak interpolation operators based on classical trigonometric interpolation which work well also in the quasi-Banach situation whenever the integrability in the target space satisfies $q>1$, cf. Theorem 6.3 and 6.4.

Finally, let us mention that in [3] we provide similar characterizations for non-periodic Triebel-Lizorkin spaces using time local building blocks, i.e. the Faber-Schauder system. Recently, Dinh Dũng [8] extended this idea to prove B-spline quasi-interpolant characterizations in Sobolev spaces $S_{p}^{r} W\left(\mathbb{T}^{d}\right)$.

Outline. The paper is organized as follows. Section 2 deals with the construction of univariate interpolation operators and corresponding kernels necessary for (1.10). In Section 3 we define and discuss several characterizations of Besov-Triebel-Lizorkin spaces. In Section 4 we provide tools for estimating the norms of superpositions of dyadic trigonometric polynomials in Besov-Triebel-Lizorkin spaces. Section 5 gives a proof for the sampling representations, see 
(1.14), which are used in Section 6 to analyze Smolyak's algorithm in this context. In the last section we compare these results with linear and Gelfand $n$-widths. Finally, in the appendix we recall some basic facts and known results on maximal inequalities, Fourier transform, Fourier series, exponential sums and $n$-widths.

Notation. As usual $\mathbb{N}$ denotes the natural numbers, $\mathbb{N}_{0}:=\mathbb{N} \cup\{0\}, \mathbb{Z}$ denotes the integers, $\mathbb{R}$ the real numbers, and $\mathbb{C}$ the complex numbers. The letter $d$ is always reserved for the underlying dimension in $\mathbb{R}^{d}, \mathbb{Z}^{d}$ etc. With $\mathbb{T}^{d}$ we denote the torus represented by the interval $[-\pi, \pi]^{d}$, where opposite points are identified. Elements $\boldsymbol{x}, \boldsymbol{y}, \boldsymbol{r} \in \mathbb{R}^{d}$ are always typesetted in bold face. We denote with $\boldsymbol{x} \cdot \boldsymbol{y}$ the usual Euclidean inner product in $\mathbb{R}^{d}$. For $a \in \mathbb{R}$ we denote $a_{+}:=\max \{a, 0\}$. For $0<p \leq \infty$ and $\boldsymbol{x} \in \mathbb{R}^{d}$ we denote $|\boldsymbol{x}|_{p}:=\left(\sum_{i=1}^{d}\left|x_{i}\right|^{p}\right)^{1 / p}$ with the usual modification in the case $p=\infty$. By $\boldsymbol{x}=\left(x_{1}, \ldots, x_{d}\right)>0$ we mean that each coordinate is positive. For $\boldsymbol{j} \in \mathbb{N}_{0}^{d}$ we use the notation $\mathbf{2}^{\boldsymbol{j}}=\left(2^{j_{1}}, \ldots, 2^{j_{d}}\right), 2^{\boldsymbol{j}}=2^{j_{1}} \cdot \ldots \cdot 2^{j_{d}}$. If $X$ and $Y$ are two (quasi-)normed spaces, the (quasi-)norm of an element $x$ in $X$ will be denoted by $\|x \mid X\|$. If $T: X \rightarrow Y$ is a continuous operator we write $T \in \mathcal{L}(X, Y)$. The symbol $X \hookrightarrow Y$ indicates that the identity operator from $X$ to $Y$ is continuous. For two sequences $\left(a_{n}\right)_{n=1}^{\infty},\left(b_{n}\right)_{n=1}^{\infty} \subset \mathbb{R}$ we write $a_{n} \lesssim b_{n}$ if there exists a constant $c>0$ such that $a_{n} \leq c b_{n}$ for all $n$. We will write $a_{n} \asymp b_{n}$ if $a_{n} \lesssim b_{n}$ and $b_{n} \lesssim a_{n}$ and use the Landau symbol $\left(a_{n}\right)_{n}=o\left(\left(b_{n}\right)_{n}\right): \Longleftrightarrow \lim _{n \rightarrow \infty} a_{n} / b_{n}=0$. In addition, we use the following notation $[d]:=\{1, \ldots, d\}, \mathbb{Z}^{d}(e):=\left\{\boldsymbol{k} \in \mathbb{Z}^{d}: k_{i}=0: \quad i \notin e\right\}, \mathbb{N}_{0}^{d}(e):=\left\{\boldsymbol{k} \in \mathbb{N}_{0}^{d}: k_{i}=0: \quad i \notin e\right\}$ where $e \subset[d], \sigma_{p}:=\max \left\{0, \frac{1}{p}-1\right\}, \sigma_{p, \theta}:=\max \left\{0, \frac{1}{p}-1, \frac{1}{\theta}-1\right\}$. For $\boldsymbol{\ell}, \boldsymbol{j} \in \mathbb{Z}^{d}, \boldsymbol{s} \in \mathbb{R}^{d}$ we use the notation $\ell>a: \Longleftrightarrow \ell_{i}>a$ for all $i \in[d]$ and $\ell>j: \Longleftrightarrow \ell_{i}>j_{i}$ for all $i \in[d]$. For $\Omega \subset \mathbb{R}^{d}$ the set of all bounded and continuous functions $f: \Omega \rightarrow \mathbb{C}$ is denoted by $C(\Omega)$. We denote by $L_{p}(\Omega), 0<p \leq \infty$, the space of all measurable functions $f: \Omega \rightarrow \mathbb{C}$ where $\|f\|_{p}:=\left(\int_{\Omega}|f(\boldsymbol{x})|^{p} d \boldsymbol{x}\right)^{1 / p}$ is finite (with the usual modification if $p=\infty$ ). For $f \in L_{1}\left(\mathbb{R}^{d}\right)$ and $\boldsymbol{x}, \boldsymbol{\xi} \in \mathbb{R}^{d}$ we define the Fourier transform and its inverse by

$$
\mathcal{F} f(\boldsymbol{\xi}):=\frac{1}{(2 \pi)^{\frac{d}{2}}} \int_{\mathbb{R}^{d}} f(\boldsymbol{x}) e^{-i \boldsymbol{\xi} \cdot \boldsymbol{x}} d \boldsymbol{x} \quad \text { and } \quad \mathcal{F}^{-1} f(\boldsymbol{x}):=\frac{1}{(2 \pi)^{\frac{d}{2}}} \int_{\mathbb{R}^{d}} f(\boldsymbol{x}) e^{i \boldsymbol{\xi} \cdot \boldsymbol{x}} d \boldsymbol{\xi} .
$$

For $f \in L_{1}\left(\mathbb{T}^{d}\right)$ the $\boldsymbol{k}$-th Fourier coefficient is defined by $\hat{f}(\boldsymbol{k}):=1 /(2 \pi)^{d} \int_{\mathbb{T}^{d}} f(\boldsymbol{x}) e^{-i \boldsymbol{k} \cdot \boldsymbol{x}} d \boldsymbol{x}$. The convolution is always defined as $f * g(\boldsymbol{x}):=\int f(\boldsymbol{y}) g(\boldsymbol{x}-\boldsymbol{y}) d \boldsymbol{y}$.

\section{Frequency-limited fundamental interpolants}

\subsection{Univariate fundamental interpolants}

In this section we construct univariate sampling operators of type (1.11) based on bandlimited kernels $K: \mathbb{R} \rightarrow \mathbb{C}$ with suitable decay. Here $K_{\pi, j}^{L}$ denotes the $2 \pi$-periodization of $K^{L}\left(2^{j}(\cdot)\right)$ which we will call fundamental interpolant. The following construction allows to arrange any prescribed polynomial decay (of order $L$ ) of the kernel $K$, which is crucial for our analysis. In addition the operator $I_{j}^{L}$ is supposed to reproduce trigonometric polynomials of a degree related to $\asymp 2^{j}$. The sampling kernels we study are constructed from a finite product of dilated sinc functions. As a starting point we define for $L \in \mathbb{N}$,

$$
K^{L}(x):=\prod_{\ell=1}^{L} \operatorname{sinc}\left(2^{-\ell} x\right), \quad x \in \mathbb{R}
$$


with

$$
\operatorname{sinc}(x):=\left\{\begin{array}{lll}
\frac{\sin (x)}{x} & : & x \neq 0 \\
1 & : & \text { otherwise. }
\end{array}\right.
$$

The next step is a $2 \pi$-periodization of dyadic dilations of $K^{L}(x)$ given by

$$
K_{\pi, j}^{L}(x):=\sum_{k=-\infty}^{\infty} K^{L}\left(2^{j}(x+2 \pi k)\right) .
$$

In case $L=1$ the summation in (2.1) does not make sense. Instead we use a direct definition

$$
K_{\pi, j}^{1}(x):=2^{-j} \mathcal{D}_{j}^{1}(x):=2^{-j} \sum_{k=-2^{j-1}}^{2^{j-1}-1} e^{i k x} .
$$

This kernel represents an exception and requires some extra attention in the whole paper. It is a convenient modification of the classical Dirichlet kernel that provides a nested set of zeros as $j$ increases, cf. [12, (2.6)]. For $j \in \mathbb{N}_{0}$ we define the interpolation operator

$$
I_{j}^{L}[f](x):=\sum_{u=-2^{j-1}}^{2^{j-1}-1} f\left(\frac{2 \pi u}{2^{j}}\right) K_{\pi, j}^{L}\left(x-\frac{2 \pi u}{2^{j}}\right),
$$

where in case $j=0$ we put $I_{0}^{L}[f](x):=f(0) K_{\pi, 0}^{L}(x)$. The kernel defined in (2.1) consists of a sum with infinitely many summands. For practical reasons such a definition is not useful. For every fixed $L \in \mathbb{N}$ we can compute an explicit representation of the kernel. Beginning from the definition we obtain the following identity

$$
K_{\pi, j}^{L}(x)=\sum_{k=-\infty}^{\infty} \prod_{\ell=1}^{L} \frac{\sin \left(2^{j-\ell}(x+2 \pi k)\right)}{2^{j-\ell}(x+2 \pi k)} .
$$

Obviously, in case $x \bmod \pi=0$ we obtain

$$
K_{\pi, j}^{L}(0)=K^{L}(0)=1 .
$$

In case $0<|x|<\pi$ an elementary calculation shows

$$
\begin{aligned}
K_{\pi, j}^{L}(x) & =\sum_{k=-\infty}^{\infty} \prod_{\ell=1}^{L} \frac{\sin \left(2^{j-\ell} x\right) \cos \left(2^{\ell+j+1} \pi k\right)+\sin \left(2^{\ell+j+1} \pi k\right) \cos \left(2^{j-\ell} x\right)}{2^{j-\ell}(x+2 \pi k)} \\
& =\sum_{k=-\infty}^{\infty} \prod_{\ell=1}^{L} \frac{\sin \left(2^{j-\ell} x\right)}{2^{j-\ell}(x+2 \pi k)}=\frac{\sin \left(2^{j-1} x\right) \ldots \sin \left(2^{j-L} x\right)}{2^{j L} 2^{-\frac{(L+1) L}{2}}} \sum_{k=-\infty}^{\infty} \frac{1}{(x+2 \pi k)^{L}} .
\end{aligned}
$$

Using the so-called Herglotz-trick (cf. [1]) we find

$$
\frac{1}{2} \cot \left(\frac{x}{2}\right)=\sum_{k=-\infty}^{\infty} \frac{1}{x+2 \pi k}
$$

Taking $L-1$ derivatives yields

$$
\left[\frac{1}{2} \cot \left(\frac{\dot{2}}{2}\right)\right]^{(L-1)}(x)=(-1)^{L-1}(L-1) ! \sum_{k=-\infty}^{\infty} \frac{1}{(x+2 \pi k)^{L}} .
$$


Computing $\left[\frac{1}{2} \cot \left(\frac{\dot{\overline{2}}}{)}\right]^{(L-1)}\right.$ and inserting this identity in (2.2) gives us a closed representation of the kernel $K_{\pi, j}^{L}(x)$. For $L=2$ and $L=3$ we obtain the explicit representations

$$
K_{\pi, j}^{2}(x)= \begin{cases}\frac{2 \sin \left(2^{j-1} x\right) \sin \left(2^{j-2} x\right)}{2^{2 j} \sin ^{2}\left(\frac{x}{2}\right)} & : \quad x \bmod 2 \pi \neq 0 \\ 1 & : \quad \text { otherwise }\end{cases}
$$

and

$$
K_{\pi, j}^{3}(x)=\left\{\begin{array}{lll}
8 \frac{\sin \left(2^{j-1} x\right) \sin \left(2^{j-2} x\right) \sin \left(2^{j-3} x\right) \cos \left(\frac{x}{2}\right)}{2^{3 j} \sin ^{3}\left(\frac{x}{2}\right)} & : & x \bmod 2 \pi \neq 0, \\
1 & : & \text { otherwise }
\end{array}\right.
$$

Remark 2.1. $K^{L}, L>1$, consists of products of dilated sinc functions. The convolution property of the Fourier transform yields

$$
K^{L}(x)=\prod_{\ell=1}^{L} \operatorname{sinc}\left(2^{-\ell} x\right)=\sqrt{2 \pi} \mathcal{F}\left[\chi_{\left[-2^{-1}, 2^{-1}\right]} * \ldots * 2^{L-1} \chi_{\left[-2^{-L}, 2^{-L}\right]}(\cdot)\right](x)
$$

Altogether $\mathcal{F} K^{L}$ is a locally supported $L-2$ times continuously differentiable function fulfilling

$$
\mathcal{F} K^{L}(\xi)= \begin{cases}\sqrt{2 \pi} & :|\xi| \leq \frac{1}{2^{L}} \\ 0 & :|\xi| \geq 1-\frac{1}{2^{L}}\end{cases}
$$

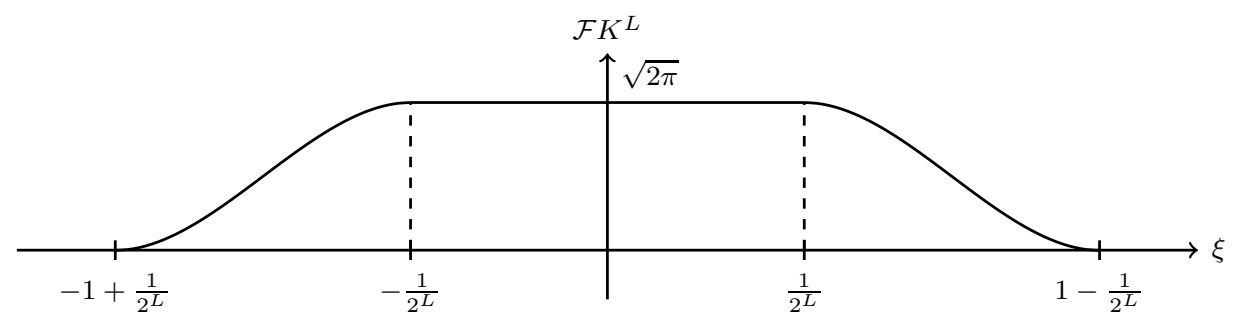

Lemma 2.2. Let $L \geq 1, j \in \mathbb{N}_{0}$ and $f \in C(\mathbb{T})$.

(i) Then for $\ell \in \mathbb{Z}$

$$
\widehat{I_{j}^{L}[f]}(\ell)=\frac{1}{\sqrt{2 \pi}} \mathcal{F} K^{L}\left(\frac{\ell}{2^{j}}\right) \sum_{u=-2^{j-1}}^{2^{j-1}-1} f\left(\frac{2 \pi u}{2^{j}}\right) e^{-i \frac{2 \pi u}{2^{j}} \ell}
$$

holds true.

(ii) If additionally $\sum_{\ell \in \mathbb{Z}}|\widehat{f}(\ell)|<\infty$ is fulfilled. Then

$$
\widehat{I_{j}^{L}[f]}(\ell)=\frac{1}{\sqrt{2 \pi}} \mathcal{F} K^{L}\left(\frac{\ell}{2^{j}}\right) \sum_{k \in \mathbb{Z}} \widehat{f}\left(\ell+2^{j} k\right)
$$

holds. 
Proof. We compute the $\ell$-th Fourier coefficient of $f$ and obtain by the translation property the following identity

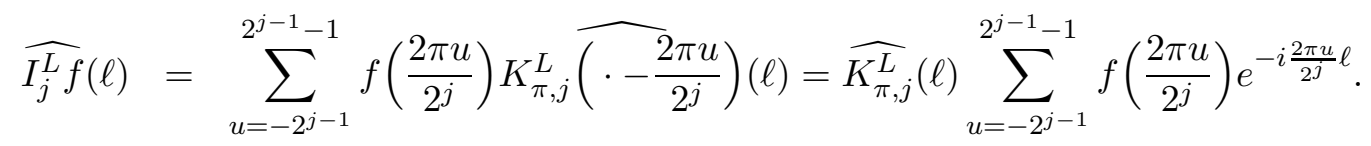

Lemma A.2 together with the dilation property of the Fourier transform yields

$$
\widehat{I_{j}^{L} f}(\ell)=\frac{1}{\sqrt{2 \pi} 2^{j}} \mathcal{F} K^{L}\left(\frac{\ell}{2^{j}}\right) \sum_{u=-2^{j-1}}^{2^{j-1}-1} f\left(\frac{2 \pi u}{2^{j}}\right) e^{-i \frac{2 \pi u}{2^{j}} \ell} .
$$

If the Fourier coefficients are absolutely summable we get

$$
\widehat{I_{j}^{L} f}(\ell)=\frac{1}{\sqrt{2 \pi} 2^{j}} \mathcal{F} K^{L}\left(\frac{\ell}{2^{j}}\right) \sum_{u=-2^{j-1}}^{2^{j-1}-1}\left(\sum_{k \in \mathbb{Z}} \widehat{f}(k) e^{i k \frac{2 \pi u}{2^{j}}}\right) e^{-i \frac{2 \pi u}{2^{j}} \ell} .
$$

Interchanging the order of summation yields

$$
\widehat{I_{j}^{L} f}(\ell)=\frac{1}{\sqrt{2 \pi} 2^{j}} \mathcal{F} K^{L}\left(\frac{\ell}{2^{j}}\right) \sum_{k \in \mathbb{Z}} \widehat{f}(k) \sum_{u=-2^{j-1}}^{2^{j-1}-1} e^{i \frac{2 \pi u}{2^{j}}(k-\ell)} .
$$

The formula for geometric partial sums tells us

$$
\sum_{u=-2^{j-1}}^{2^{j-1}-1} e^{i \frac{2 \pi u}{2^{j}}(k-\ell)}=\left\{\begin{array}{lll}
2^{j} & : & k-\ell \bmod 2^{j}=0 \\
0 & : & \text { otherwise }
\end{array}\right.
$$

Finally, we obtain

$$
\widehat{I_{j}^{L} f}(\ell)=\frac{1}{\sqrt{2 \pi}} \mathcal{F} K^{L}\left(\frac{\ell}{2^{j}}\right) \sum_{k \in \mathbb{Z}} \widehat{f}\left(\ell+2^{j} k\right) .
$$

Definition 2.3. We define for $j, L \in \mathbb{N}_{0}$ the dyadic blocks

$$
\mathcal{P}_{j}^{L}:=\left\{k \in \mathbb{Z}:|k| \leq \frac{1}{2^{L}} 2^{j}\right\} .
$$

Additionally, we denote the set of trigonometric polynomials with frequencies in $\mathcal{P}_{j}^{L}$ by

$$
\mathcal{T}_{j}^{L}:=\operatorname{span}\left\{e^{i k x}: k \in \mathcal{P}_{j}^{L}\right\} .
$$

Corollary 2.4. Let $L \in \mathbb{N}$ and $f \in C(\mathbb{T})$.

(i) Then it holds $I_{j}^{L}[f] \in \mathcal{T}_{j}^{0}$.

(ii) If additionally $f \in \mathcal{T}_{j}^{L}$ then $I_{j}^{L}[f]=f$. 
Proof. Assertion (i) is an easy consequence of (2.5) together with the support properties of $K^{L}$. For assertion (ii) we may use

$$
\widehat{I_{j}^{L}[f]}(\ell)=\frac{1}{\sqrt{2 \pi}} \mathcal{F} K^{L}\left(\frac{\ell}{2^{j}}\right) \sum_{k \in \mathbb{Z}} \widehat{f}\left(\ell+2^{j} k\right)
$$

which equals $\widehat{f}(\ell)$ for all $\ell$ if $f \in \mathcal{T}_{j}^{L}$.

The next lemma provides the reason for calling $K_{\pi, j}^{L}$ a fundamental interpolant for the equidistant grid $\mathcal{G}_{j}^{1}:=\left\{\frac{-2 \pi 2^{j-1}}{2^{j}}, \ldots, \frac{2 \pi\left(2^{j-1}-1\right)}{2^{j}}\right\}$.

Lemma 2.5. Let $f \in C(\mathbb{T})$ and $L \geq 1$. Then

$$
f\left(\frac{2 \pi u}{2^{j}}\right)=I_{j}^{L} f\left(\frac{2 \pi u}{2^{j}}\right) \quad, \quad u \in\left\{-2^{j-1}, \ldots, 2^{j-1}-1\right\} .
$$

Proof. Obviously, it is sufficient to proof

$$
K_{\pi, j}^{L}\left(\frac{2 \pi u}{2^{j}}\right)=\delta_{0, u}
$$

In case $L=1$ this is a trivial consequence of (1.12). In case $L>1$ this we have according to our definition for $u \in\left\{-2^{j-1}, \ldots, 2^{j-1}-1\right\}$

$$
K_{\pi, j}^{L}\left(\frac{2 \pi u}{2^{j}}\right)=\sum_{k=-\infty}^{\infty} K^{L}\left(2 \pi\left(u+2^{j} k\right)\right)=\delta_{0, u} .
$$

Lemma 2.6. Let $j \in \mathbb{N}_{0}$ and $L>1$. Then there are constants $C, C^{*}>0$ (independent of $x$ and $j$ ) such that

$$
\left|K_{\pi, j}^{L}(x)\right| \leq C \min \left\{\frac{1}{\left|2^{j} x\right|^{L}}, 1\right\} \leq C^{*} \frac{1}{\left(1+2^{j}|x|\right)^{L}}
$$

holds for all $x \in[-\pi, \pi]$.

Proof. The second inequality of the chain is trivial. We prove the first one. Starting for $x \in[-\pi, \pi]$ the estimate with

$$
\begin{aligned}
\left|K_{\pi, j}^{L}(x)\right| & =\left|\sum_{k=-\infty}^{\infty} K^{L}\left(2^{j}(x+2 \pi k)\right)\right| \leq\left|K^{L}\left(2^{j} x\right)\right|+\sum_{|k|>0}\left|K^{L}\left(2^{j}(x+2 \pi k)\right)\right| \\
& \lesssim \prod_{\ell=1}^{L}\left|\operatorname{sinc}\left(2^{j-\ell} x\right)\right|+\sum_{|k|>0} \frac{1}{2^{j L}|x+2 \pi k|^{L}} .
\end{aligned}
$$

Clearly, the first summand is uniformly bounded. Estimating the second summand in (2.7) we use the fact that $|x| \leq \pi$ implies $|2 \pi k+x| \geq|\pi k|$ for every integer $k \in \mathbb{Z}$ and obtain

$$
\sum_{|k|>0} \frac{1}{2^{j L}|x+2 \pi k|^{L}} \leq \frac{1}{2^{j L} \pi^{L}} \sum_{|k|>0} \frac{1}{|k|^{L}},
$$


which is known to be finite for $L \geq 2$. Using $|x| \leq \pi$ yields

$$
\sum_{|k|>0} \frac{1}{2^{j L}|x+2 \pi k|^{L}} \lesssim \frac{1}{2^{j L}|x|^{L}}
$$

Considering again the first summand in (2.7) gives

$$
\prod_{\ell=1}^{L}\left|\operatorname{sinc}\left(2^{j-\ell} x\right)\right| \leq \frac{1}{2^{(L+1) \frac{L}{2}}} \frac{1}{2^{j L}|x|^{L}}
$$

which concludes the proof.

\subsection{Multivariate interpolation}

Based on the univariate interpolation scheme from the previous subsection we are now able to define the building blocks used for the Smolyak algorithm, cf. (1.3),

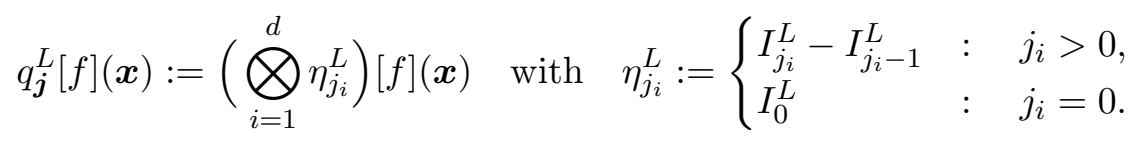

We may write $q_{j}^{L}[f]$ as follows

$$
q_{j}^{L}[f]=\sum_{\boldsymbol{b} \in\{-1,0\}^{d}} \varepsilon_{\boldsymbol{b}} I_{\boldsymbol{j}+\boldsymbol{b}}^{L}[f]
$$

with suitable signs $\varepsilon_{\boldsymbol{b}}$. The definition of the operators $I_{\boldsymbol{j}+\boldsymbol{b}}^{L}[f](\boldsymbol{x})$ requires some more notation.

$$
\boldsymbol{x}_{\boldsymbol{u}}^{j}=\left(x_{u_{1}}^{j_{1}}, \ldots, x_{u_{d}}^{j_{d}}\right) \quad, \quad \boldsymbol{u} \in \mathbb{Z}^{d}
$$

where $x_{u}^{j}=2 \pi u / 2^{j}$ for $u \in \mathbb{Z}$. For $\boldsymbol{x} \in \mathbb{R}^{d}$ let further

$$
A_{\boldsymbol{j}}(\boldsymbol{x}):=A_{j_{1}}\left(x_{1}\right) \times \ldots \times A_{j_{d}}\left(x_{d}\right)
$$

with $A_{j}(x)=\left\{u \in \mathbb{Z}: x_{u}^{j} \in[x-\pi, x+\pi)\right\}$ and put $A_{j}:=A_{j}(\mathbf{0})$. We further let

$$
K_{\pi^{d}, \boldsymbol{j}}^{L}:=\prod_{i=1}^{d} K_{\pi, j_{i}}^{L}\left(x_{i}\right)
$$

and define the tensorized interpolation operator by

$$
I_{j}^{L}[f]=\sum_{\boldsymbol{u} \in A_{j}} f\left(\boldsymbol{x}_{\boldsymbol{u}}^{\boldsymbol{j}}\right) K_{\pi^{d}, \boldsymbol{j}}^{L}\left(\boldsymbol{x}-\boldsymbol{x}_{u}^{\boldsymbol{j}}\right)
$$

Lemma 2.7. Let $\Delta \subset \mathbb{N}_{0}^{d}$ be a solid finite set meaning that $\boldsymbol{j} \in \Delta$ and $\boldsymbol{k} \leq \boldsymbol{j}$ implies $\boldsymbol{k} \in \Delta$. Then $\sum_{\boldsymbol{j} \in \Delta} q_{\boldsymbol{j}}^{L}[f]$ reproduces trigonometric polynomials with frequencies in

$$
\mathcal{H}_{\Delta}^{L}:=\bigcup_{j \in \Delta} \mathcal{P}_{j}^{L}
$$


Proof. We refer to [2, Lem. 6.1].

Lemma 2.8. Let $\Delta \subset \mathbb{N}_{0}^{d}$ be a solid finite set (i.e. $\boldsymbol{k} \leq \boldsymbol{j}$ and $\boldsymbol{j} \in \Delta$ implies $\boldsymbol{k} \in \Delta$ ). Then $T_{\Delta}^{L} f:=\sum_{\boldsymbol{j} \in \Delta} q_{\boldsymbol{j}}^{L}[f]$ interpolates $f$ on the grid

$$
G_{\Delta}^{d}:=\bigcup_{j \in \Delta}\left\{\boldsymbol{x}_{\boldsymbol{u}}^{\boldsymbol{j}}: \boldsymbol{u} \in A_{\boldsymbol{j}}\right\}
$$

that means

$$
f(\boldsymbol{x})=T_{\Delta}^{L} f(\boldsymbol{x})
$$

for all $\boldsymbol{x} \in G_{\Delta}^{d}$.

Proof. The interpolation property of the univariate operator $I_{j}^{L}$ in Lemma 2.5 immediately gives an interpolation property of the multivariate sampling operator $I_{\left(m_{1}, \ldots, m_{d}\right)}^{L}$ on a "full grid" $\mathcal{G}_{\{\boldsymbol{j} \leq \boldsymbol{m}\}}^{d}$. Choosing $\boldsymbol{m}$ such that $\Delta \subset\{\boldsymbol{j} \leq \boldsymbol{m}\}$ and arguing similar as in Lemma [37, Lem. 4.3] gives the result.

Definition 2.9. For $\boldsymbol{j} \in \mathbb{N}_{0}^{d}$ and $L \in \mathbb{N}$ we tensorize the dyadic blocks defined in (2.6) by

$$
\mathcal{P}_{j}^{L}:=\mathcal{P}_{j_{1}}^{L} \cdot \ldots \cdot \mathcal{P}_{j_{d}}^{L},
$$

and define the set of trigonometric polynomials with frequencies in $\mathcal{P}_{\boldsymbol{j}}^{L}$ by

$$
\mathcal{T}_{\boldsymbol{j}}^{L}:=\operatorname{span}\left\{e^{i \boldsymbol{k} \cdot \boldsymbol{x}}: \boldsymbol{k} \in \mathcal{P}_{\boldsymbol{j}}^{L}\right\} .
$$

Proposition 2.10. Let $L \in \mathbb{N}$ and $f \in \mathcal{T}_{\boldsymbol{\ell}}^{L}$ then $q_{\boldsymbol{j}}^{L}[f] \neq 0$ implies $\boldsymbol{\ell} \geq \boldsymbol{j}$.

Proof. The proof follows immediately from the definition of $q_{\boldsymbol{j}}^{L}[f]$ in (2.8) and the univariate reproduction property in Corollary 2.4.

\section{Besov-Triebel-Lizorkin spaces of mixed smoothness}

In this section we give the classical Fourier analytical definition of periodic Triebel-Lizorkin spaces $S_{p, \theta}^{r} F\left(\mathbb{T}^{d}\right)$ and Besov spaces $S_{p, \theta}^{r} B\left(\mathbb{T}^{d}\right)$ with dominating mixed smoothness. We start introducing vector-valued Lebesgue spaces.

Definition 3.1. We define for $0<p, \theta \leq \infty$ the spaces $L_{p}\left(\ell_{\theta}\left(\mathbb{N}_{0}^{d}\right)\right)$ and $\ell_{\theta}\left(L_{p}\left(\mathbb{T}^{d}\right)\right)$ as the space of all sequences of functions $\left(f_{\boldsymbol{j}}\right)_{\boldsymbol{j} \in \mathbb{N}_{0}^{d}} \subset L_{p}\left(\mathbb{T}^{d}\right)$ with finite (quasi)-norm

$$
\left\|f_{\boldsymbol{j}} \mid L_{p}\left(\mathbb{T}^{d}, \ell_{\theta}\left(\mathbb{N}_{0}^{d}\right)\right)\right\|:= \begin{cases}\left\|\left(\sum_{\boldsymbol{j} \in \mathbb{N}_{0}^{d}}\left|f_{\boldsymbol{j}}\right|^{\theta}\right)^{\frac{1}{\theta}}\right\|_{p} & : \quad 0<\theta<\infty, \\ \left\|\sup _{\boldsymbol{j} \in \mathbb{N}_{0}^{d}}\left|f_{\boldsymbol{j}}\right|\right\|_{p} & : \quad \theta=\infty,\end{cases}
$$

and

$$
\left\|f_{\boldsymbol{j}} \mid \ell_{\theta}\left(L_{p}\left(\mathbb{T}^{d}\right)\right)\right\|:= \begin{cases}\left(\sum_{\boldsymbol{j} \in \mathbb{N}_{0}^{d}}\left\|f_{\boldsymbol{j}}\right\|_{p}^{\theta}\right)^{\frac{1}{\theta}} & : \quad 0<\theta<\infty \\ \sup _{\boldsymbol{j} \in \mathbb{N}_{0}^{d}}\left\|f_{\boldsymbol{j}}\right\|_{p} & : \quad \theta=\infty\end{cases}
$$

respectively. 
For $0<p, \theta \leq \infty$ the (quasi-)norms $\left\|\cdot \mid L_{p}\left(\mathbb{T}^{d}, \ell_{\theta}\left(\mathbb{N}_{0}^{d}\right)\right)\right\|$ and $\left\|\cdot \mid \ell_{\theta}\left(L_{p}\left(\mathbb{T}^{d}\right)\right)\right\|$ fulfill a $\mu$-triangle inequality with $\mu=\min \{p, \theta, 1\}$.

Definition 3.2. A system $\varphi=\left(\varphi_{j}\right)_{j=0}^{\infty} \subset C_{0}^{\infty}(\mathbb{R})$ belongs to the class $\Phi(\mathbb{R})$ if and only if

(i) It exists $A>0$ such that $\operatorname{supp} \varphi_{0} \subset[-A, A]$.

(ii) There are constants $0<B<C$, such that $\operatorname{supp} \varphi_{j} \subset\left\{\xi \in \mathbb{R}: B 2^{j} \leq|\xi| \leq C 2^{j}\right\}$.

(iii) For all $r \in \mathbb{N}_{0}$ holds

$$
\sup _{\xi \in \mathbb{R}, j \in \mathbb{N}_{0}} 2^{j r}\left|D^{r} \varphi_{j}(\xi)\right| \leq c_{r}<\infty \text { and }
$$

$$
\sum_{j=0}^{\infty} \varphi_{j}(\xi)=1 .
$$

Because of (iv) in Definition 3.2 we obtain the following decomposition of $f \in D^{\prime}\left(\mathbb{T}^{d}\right)$. Here, $D^{\prime}\left(\mathbb{T}^{d}\right)$ denotes the space of distributions on $\mathbb{T}^{d}$. For more details on periodic distributions we refer to [32, Section 3.2]. Let

$$
\delta_{\boldsymbol{j}}[f](\boldsymbol{x}):=\sum_{\boldsymbol{k} \in \mathbb{Z}^{d}} \varphi_{j_{1}}\left(k_{1}\right) \cdot \ldots \cdot \varphi_{j_{d}}\left(k_{d}\right) \hat{f}(\boldsymbol{k}) e^{i \boldsymbol{k} \cdot \boldsymbol{x}}
$$

be a trigonometric polynomial. Then it holds

$$
f=\sum_{\boldsymbol{j} \in \mathbb{N}_{0}^{d}} \delta_{j}[f]
$$

with convergence in $D^{\prime}\left(\mathbb{T}^{d}\right)$. We introduce the function spaces $S_{p, \theta}^{\boldsymbol{r}} F\left(\mathbb{T}^{d}\right)$ and $S_{p, \theta}^{\boldsymbol{r}} B\left(\mathbb{T}^{d}\right)$ using these Fourier-analytic building blocks.

Definition 3.3. Let $\varphi=\left\{\varphi_{j}(x)\right\}_{j=0}^{\infty} \in \Phi(\mathbb{R})$, and $\boldsymbol{r} \in \mathbb{R}^{d}$. Let further

(i) $0<p<\infty$ and $0<\theta \leq \infty$. Then

$$
S_{p, \theta}^{r} F\left(\mathbb{T}^{d}\right):=\left\{f \in D^{\prime}\left(\mathbb{T}^{d}\right):\left\|f \mid S_{p, \theta}^{r} F\left(\mathbb{T}^{d}\right)\right\|<\infty\right\},
$$

where $\left\|f\left|S_{p, \theta}^{r} F\left(\mathbb{T}^{d}\right)\|:=\| 2^{r \cdot j} \delta_{j}[f]\right| L_{p}\left(\ell_{\theta}\left(\mathbb{N}_{0}^{d}\right)\right)\right\|$.

(ii) $0<p, \theta \leq \infty$. Then

$$
S_{p, \theta}^{r} B\left(\mathbb{T}^{d}\right):=\left\{f \in D^{\prime}\left(\mathbb{T}^{d}\right):\left\|f \mid S_{p, q}^{r} B\left(\mathbb{T}^{d}\right)\right\|<\infty\right\},
$$

where $\left\|f\left|S_{p, \theta}^{r} B\left(\mathbb{T}^{d}\right)\|:=\| 2^{r \cdot j} \delta_{j}[f]\right| \ell_{\theta}\left(L_{p}\left(\mathbb{N}_{0}^{d}\right)\right)\right.$.

Remark 3.4. (i) Different resolutions of unity $\varphi, \psi \in \Phi(\mathbb{R})$ used in (3.1) generate equivalent norms in $S_{p, \theta}^{r} F\left(\mathbb{T}^{d}\right)$ and $S_{p, \theta}^{r} B\left(\mathbb{T}^{d}\right)$, respectively.

(ii) When replacing the smooth dyadic decomposition of unity by a less regular window function (like de la Vallée Poussin means) we encounter the same condition on the decay $L$ of the convolution kernel as we observe for the sampling representations below. It is open whether this is optimal. However, taking [4, 33, 34] into account we strongly conjecture the optimality. 
(iii) It was proved in [45, Thms. 3.8.1, 3.8.2] that B-spaces defined via modulus of continuity (bounded mixed difference, see for instance [5, 6, 42]) and the Fourier-analytical approach in the present paper coincide in case $p \geq 1$ and $\boldsymbol{r}>0$. For $p<1$ one does not even know the equivalence if $\boldsymbol{r}>\sigma_{p}$. In Theorem [45, Thm. 3.8.3] we need $\boldsymbol{r}>1 / p$, see also [32, Thm. 2.3.4/2, Rem. 2.3.4/2]. In case $0<\boldsymbol{r} \leq \sigma_{p}$ both approaches may yield different spaces.

In case $d=1$ the concepts of dominating mixed smoothness and isotropic smoothness coincide. We use the notation

$$
F_{p, \theta}^{r}(\mathbb{T}):=S_{p, \theta}^{r} F(\mathbb{T}) \quad \text { and } \quad B_{p, \theta}^{r}(\mathbb{T}):=S_{p, \theta}^{r} B(\mathbb{T}) .
$$

In case $\theta=2$ with $1<p<\infty$ the space $S_{p, \theta}^{r} F\left(\mathbb{T}^{d}\right)$ coincides with the Sobolev space of dominating mixed smoothness $S_{p}^{r} W\left(\mathbb{T}^{d}\right)$ including $L_{p}\left(\mathbb{T}^{d}\right)$ if $\boldsymbol{r}=0 . \quad S_{p}^{r} W\left(\mathbb{T}^{d}\right)$ is classically normed by

$$
\left\|f \mid S_{p}^{\boldsymbol{r}} W\left(\mathbb{T}^{d}\right)\right\|^{\prime}:=\left\|\sum_{\boldsymbol{k} \in \mathbb{Z}^{d}} \widehat{f}(\boldsymbol{k}) \prod_{i=1}^{d}\left(1+\left|k_{i}\right|^{2}\right)^{\frac{r_{i}}{2}} e^{i \boldsymbol{k} \cdot \boldsymbol{x}}\right\|_{p} .
$$

We state the following embedding results without proof. For a reference see [32, 47] and [17]. For a complete history of the non-trivial embedding in Lemma 3.6 we refer to [12, Remark $3.8]$.

Lemma 3.5. (i) Let $0<p \leq \infty$ (F-case: $p<\infty), 0<\theta \leq \infty, \boldsymbol{r}>\sigma_{p}$. Then

$$
S_{p, \theta}^{r} F\left(\mathbb{T}^{d}\right) \hookrightarrow L_{\max \{p, 1\}}\left(\mathbb{T}^{d}\right) \quad \text { and } \quad S_{p, \theta}^{r} B\left(\mathbb{T}^{d}\right) \hookrightarrow L_{\max \{p, 1\}}\left(\mathbb{T}^{d}\right),
$$

which means $S_{p, \theta}^{\boldsymbol{r}} F\left(\mathbb{T}^{d}\right)$ and $S_{p, \theta}^{\boldsymbol{r}} B\left(\mathbb{T}^{d}\right)$ consist of regular distributions that allow an interpretation as functions.

(ii) Let $0<p \leq \infty$ (F-case: $p<\infty), 0<\theta \leq \infty, \boldsymbol{r}>\frac{1}{p}$. Then

$$
S_{p, \theta}^{r} F\left(\mathbb{T}^{d}\right) \hookrightarrow C\left(\mathbb{T}^{d}\right) \quad \text { and } \quad S_{p, \theta}^{r} B\left(\mathbb{T}^{d}\right) \hookrightarrow C\left(\mathbb{T}^{d}\right),
$$

which means that we find in every equivalence class of $S_{p, \theta}^{r} F\left(\mathbb{T}^{d}\right)$ and $S_{p, \theta}^{r} B\left(\mathbb{T}^{d}\right)$ a unique continuous representative making discrete point evaluations possible.

(iii) Let $0<q<p \leq \infty$ (F-case: $p<\infty), 0<\theta \leq \infty$ and $\boldsymbol{r} \in \mathbb{R}^{d}$. Then

$$
S_{p, \theta}^{r} F\left(\mathbb{T}^{d}\right) \hookrightarrow S_{q, \theta}^{r} F\left(\mathbb{T}^{d}\right) \quad \text { and } \quad S_{p, \theta}^{r} B\left(\mathbb{T}^{d}\right) \hookrightarrow S_{q, \theta}^{r} B\left(\mathbb{T}^{d}\right) .
$$

(iv) Let $0<p \leq \infty$ (F-case: $p<\infty), 0<\theta_{1}<\theta_{2} \leq \infty$ and $\boldsymbol{r} \in \mathbb{R}^{d}$. Then

$$
S_{p, \theta_{1}}^{r} F\left(\mathbb{T}^{d}\right) \hookrightarrow S_{p, \theta_{2}}^{r} F\left(\mathbb{T}^{d}\right) \quad \text { and } \quad S_{p, \theta_{1}}^{r} B\left(\mathbb{T}^{d}\right) \hookrightarrow S_{p, \theta_{2}}^{r} B\left(\mathbb{T}^{d}\right) .
$$

(v) Let $0<p<\infty, 0<\theta \leq \infty$ and $\boldsymbol{r} \in \mathbb{R}^{d}$. Then

$$
S_{p, \min \{p, \theta\}}^{r} B\left(\mathbb{T}^{d}\right) \hookrightarrow S_{p, \theta}^{r} F\left(\mathbb{T}^{d}\right) \hookrightarrow S_{p, \max \{p, \theta\}}^{r} B\left(\mathbb{T}^{d}\right)
$$


(vi) Let $0<p \leq \infty(F: p<\infty), 0<\theta, \nu \leq \infty$ and $\boldsymbol{r}_{1}, \boldsymbol{r}_{2} \in \mathbb{R}^{d}$ with $\boldsymbol{r}_{1}>\boldsymbol{r}_{2}$ Then

$$
S_{p, \theta}^{\boldsymbol{r}_{1}} F\left(\mathbb{T}^{d}\right) \hookrightarrow S_{p, \nu}^{\boldsymbol{r}_{2}} F\left(\mathbb{T}^{d}\right) \quad \text { and } \quad S_{p, \theta}^{\boldsymbol{r}_{1}} B\left(\mathbb{T}^{d}\right) \hookrightarrow S_{p, \nu}^{\boldsymbol{r}_{2}} B\left(\mathbb{T}^{d}\right)
$$

(vii) Let $0<p<q<\infty, 0<\theta, \nu \leq \infty$ and $\boldsymbol{r}_{1}, \boldsymbol{r}_{2} \in \mathbb{R}^{d}$ with $\boldsymbol{r}_{1}>\boldsymbol{r}_{2}$ fulfilling

$$
\boldsymbol{r}_{1}-\frac{1}{p}=\boldsymbol{r}_{2}-\frac{1}{q}
$$

Then

$$
S_{p, \theta}^{\boldsymbol{r}_{1}} F\left(\mathbb{T}^{d}\right) \hookrightarrow S_{q, \nu}^{\boldsymbol{r}_{2}} F\left(\mathbb{T}^{d}\right) \quad \text { and } \quad S_{p, \theta}^{\boldsymbol{r}_{1}} B\left(\mathbb{T}^{d}\right) \hookrightarrow S_{q, \theta}^{\boldsymbol{r}_{2}} B\left(\mathbb{T}^{d}\right) .
$$

Observe that, in contrast to the diagonal Besov embedding in Lemma 3.5, (vi), the fine index $\theta$ and $\nu$ play no role for the $F$-case.

Lemma 3.6 (Jawerth-Franke embedding). Let $0<p<q \leq \infty, 0<\theta \leq \infty, \boldsymbol{r}_{1}, \boldsymbol{r}_{2} \in \mathbb{R}^{d}$ such that

$$
\boldsymbol{r}_{1}-\frac{1}{p}=\boldsymbol{r}_{2}-\frac{1}{q}
$$

is fulfilled.

(i) Then

$$
S_{p, \theta}^{\boldsymbol{r}_{1}} F\left(\mathbb{T}^{d}\right) \hookrightarrow S_{q, p}^{\boldsymbol{r}_{2}} B\left(\mathbb{T}^{d}\right)
$$

(ii) If additionally $q<\infty$ then

$$
S_{p, q}^{\boldsymbol{r}_{1}} B\left(\mathbb{T}^{d}\right) \hookrightarrow S_{q, \theta}^{\boldsymbol{r}_{2}} F\left(\mathbb{T}^{d}\right)
$$

Definition 3.7. For a univariate function $f: \mathbb{T} \rightarrow \mathbb{C}$ we introduce the following difference operator. Let $h \in \mathbb{R}$ and $m \in \mathbb{N}$. Then for $x \in \mathbb{T}^{d}$

$$
\Delta_{h}^{m} f(x):=\sum_{k=0}^{m}(-1)^{m-k}\left(\begin{array}{c}
m \\
k
\end{array}\right) f(x+k h) .
$$

Definition 3.8. For a multivariate function $f: \mathbb{T}^{d} \rightarrow \mathbb{C}$ and $m \in \mathbb{N}, h \in \mathbb{R}, i \in[d]$ we denote with $\Delta_{h}^{m, i} f(\boldsymbol{x})$ the operator from (3.3) applied to the $i$-th direction. This allows us to define for $e \subset[d], \boldsymbol{m} \in \mathbb{N}^{d}$ and $\boldsymbol{h} \in \mathbb{R}^{d}$ the operator

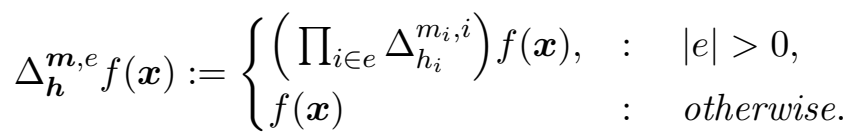

Theorem 3.9. Let $0<p<\infty, 0<\theta \leq \infty, \boldsymbol{r} \in \mathbb{R}^{d}$ and $\boldsymbol{m} \in \mathbb{N}^{d}$ such that $\sigma_{p, \theta}<\boldsymbol{r}<\boldsymbol{m}$ is fulfilled. Then

$$
\left\|f\left|S_{p, \theta}^{r} F\left(\mathbb{T}^{d}\right)\left\|\asymp \sum_{e \subset[d]}\right\| f\right| S_{p, \theta}^{r} F\left(\mathbb{T}^{d}\right)\right\|_{e, \boldsymbol{m}}
$$

holds with

$$
\left\|f \mid S_{p, \theta}^{\boldsymbol{r}} F\left(\mathbb{T}^{d}\right)\right\|_{e, \boldsymbol{m}}:=\left\|\left[\sum_{\boldsymbol{j} \in \mathbb{N}_{0}^{d}(e)} 2^{\theta \boldsymbol{r} \cdot \boldsymbol{j}}\left(\left(\prod_{i \in e} 2^{j_{i}}\right) \int_{\substack{\left|h_{i}\right| \leq 2^{-j_{i}} \\ i \in[d]}}\left|\Delta_{\boldsymbol{h}}^{\boldsymbol{m}, e} f(\cdot)\right| d \boldsymbol{h}\right)^{\theta}\right]^{\frac{1}{\theta}}\right\|_{p}
$$

and the usual modification in case $\theta=\infty$. 
Proof. We refer to [20, Theorem 3.7]. There the case for constant smoothness vector $\boldsymbol{r}=$ $(r, \ldots, r)$ has been considered. The necessary modifications are straight forward.

Theorem 3.10. Let $0<p, \theta \leq \infty, \boldsymbol{r} \in \mathbb{R}^{d}$ and $\boldsymbol{m} \in \mathbb{N}^{d}$ such that $\sigma_{p}<\boldsymbol{r}<\boldsymbol{m}$ is fulfilled. Then

$$
\left\|f\left|S_{p, \theta}^{r} B\left(\mathbb{T}^{d}\right)\left\|\asymp \sum_{e \subset[d]}\right\| f\right| S_{p, \theta}^{r} B\left(\mathbb{T}^{d}\right)\right\|_{e, m}
$$

holds with

$$
\left\|f \mid S_{p, \theta}^{r} B\left(\mathbb{T}^{d}\right)\right\|_{e, \boldsymbol{m}}:=\left[\sum_{\boldsymbol{j} \in \mathbb{N}_{0}^{d}(e)} 2^{\theta \boldsymbol{r} \cdot \boldsymbol{j}}\left\|\left(\prod_{i \in e} 2^{j_{i}}\right) \int_{\substack{\left|h_{i}\right| \leq 2^{-j_{i}} \\ i \in[d]}}\left|\Delta_{\boldsymbol{h}}^{\boldsymbol{m}, e} f(\cdot)\right| d \boldsymbol{h}\right\|_{p}^{\theta}\right]^{\frac{1}{\theta}}
$$

and the usual modification in case $\theta=\infty$.

Proof. We refer to [45, Theorem 3.7.1 and Remark 3.7.1]. There the outer sum is an integral. By discretizing this into dyadic parts one obtains the form stated above.

\section{Sums of trigonometric polynomials}

In this section we want to estimate the norm of a superposition of trigonometric polynomials

$$
f=\sum_{j \in \mathbb{N}_{0}^{d}} f_{j}
$$

where $f_{j}$ are trigonometric polynomials of degree $\asymp \mathbf{2}^{j}$. In contrast to the usual LittlewoodPaley building blocks $\delta_{\boldsymbol{j}}[f]$ which are 'almost' orthogonal, we only need to restrict the degree of the polynomial in the sequel.

As a main tool we introduce the following componentwise variant of the Hardy-Littlewood maximal operator, see [47, (1.14),(1.15)], [45, (10)] and the references therein.

Definition 4.1. Let $i \in[d]$ and $f \in L_{1}^{\text {loc }}\left(\mathbb{T}^{d}\right)$ then we define the Hardy-Littlewood maximal operator in the $i$-th direction as

$$
M_{i} f(\boldsymbol{x}):=\sup _{t>0} \frac{1}{2 t} \int_{-t}^{t}\left|f\left(x_{1}, \ldots, x_{i-1}, x_{i}+y, x_{i+1}, \ldots, x_{d}\right)\right| d y .
$$

There is a corresponding variant of the Fefferman-Stein theorem, see [45, Thm. 4.1.2] and the references therein.

Theorem 4.2. Let $1<p, q<\infty$ and $\left(f_{\boldsymbol{k}}\right)_{\boldsymbol{k}} \subset L_{p}\left(\mathbb{T}^{d}, \ell_{\theta}\right)$ and $i \in[d]$. Then we have

$$
\left\|M_{i} f_{\boldsymbol{k}}\left|L_{p}\left(\mathbb{T}^{d}, \ell_{\theta}\right)\|\lesssim\| f_{\boldsymbol{k}}\right| L_{p}\left(\mathbb{T}^{d}, \ell_{\theta}\right)\right\|
$$

Definition 4.3 (Mixed Peetre maximal function). Let $a>0$ and $\boldsymbol{b}>0$ then we define for $f \in C\left(\mathbb{T}^{d}\right)$

$$
P_{\boldsymbol{b}, a} f(\boldsymbol{x}):=\sup _{\boldsymbol{y} \in \mathbb{R}^{d}} \frac{|f(\boldsymbol{x}+\boldsymbol{y})|}{\left(1+b_{1}\left|y_{1}\right|\right)^{a} \ldots\left(1+b_{d}\left|y_{d}\right|\right)^{a}} .
$$

The mixed Peetre maximal function can be pointwise estimated by an iteration of (4.1), see [45, Lem. 3.1.1], which results in the following maximal inequalities for the mixed Peetre maximal function. 
Theorem 4.4. Let $0<p \leq \infty$ and $f$ be a trigonometric polynomial with

$$
f=\sum_{\substack{\left|k_{i}\right| \leq b_{i} \\ i=1, \ldots, d}} \hat{f}(\boldsymbol{k}) e^{i \boldsymbol{k} \cdot \boldsymbol{x}}
$$

and $a>\frac{1}{p}$. Then there is a constant $C>0$ (independent of $f$ and $\boldsymbol{b}$ ) such that

$$
\left\|P_{\boldsymbol{b}, a} f\right\|_{p} \leq C\|f\|_{p}
$$

holds.

Proof. We refer to [45, Thm. 4.1.3].

Theorem 4.5. Let $0<p<\infty, 0<\theta \leq \infty$ and $\left(f_{\boldsymbol{j}}\right)_{\boldsymbol{j} \in \mathbb{N}_{0}^{d}}$ be a sequence of trigonometric polynomials with

$$
f_{\boldsymbol{j}}=\sum_{\substack{\left|k_{i}\right| \leq b_{i}^{j} \\ i=1, \ldots, d}} \hat{f}(\boldsymbol{k}) e^{i \boldsymbol{k} \cdot \boldsymbol{x}}
$$

and $a>\max \left\{\frac{1}{p}, \frac{1}{\theta}\right\}$. Then there is a constant $C>0$ (independent of $f$ and $\boldsymbol{b}_{\boldsymbol{j}}$ ) such that

$$
\left\|P_{\boldsymbol{b}_{\boldsymbol{j}}, a} f_{\boldsymbol{j}}\left|L_{p}\left(\ell_{\theta}\right)\|\leq C\| f_{\boldsymbol{j}}\right| L_{p}\left(\ell_{\theta}\right)\right\|
$$

holds.

Proof. We refer to [45, Thm. 4.1.3].

Let us now state the main result of this subsection.

Theorem 4.6. Let $0<p<\infty, 0<\theta \leq \infty, \boldsymbol{r} \in \mathbb{R}^{d}$ with $\boldsymbol{r}>\sigma_{p, \theta}$ and $\left(f_{\boldsymbol{j}}\right)_{\boldsymbol{j} \in \mathbb{N}_{0}^{d}}$ such that $f_{\boldsymbol{j}} \in \mathcal{T}_{\boldsymbol{j}}^{0}$ and $\left\|2^{\boldsymbol{r} \cdot \boldsymbol{j}} f_{\boldsymbol{j}} \mid L_{p}\left(\ell_{\theta}\right)\right\|<\infty$. Then

(i) $\sum_{\boldsymbol{j} \in \mathbb{N}_{0}^{d}} f_{\boldsymbol{j}}$ converges unconditionally in $S_{p, \theta}^{\boldsymbol{r}} F\left(\mathbb{T}^{d}\right)$ if $\theta<\infty$ and in every $S_{p, \nu}^{\tilde{\mathbf{r}}} F\left(\mathbb{T}^{d}\right)$ with $0<\nu \leq \infty$ and $\tilde{\boldsymbol{r}}<\boldsymbol{r}$.

(ii) There is a constant $C>0$ (independent of $f$ ) such that

$$
\left\|\sum_{\boldsymbol{j} \in \mathbb{N}_{0}^{d}} f_{\boldsymbol{j}}\left|S_{p, \theta}^{\boldsymbol{r}} F\left(\mathbb{T}^{d}\right)\|\leq C\| 2^{\boldsymbol{r} \cdot \boldsymbol{j}} f_{\boldsymbol{j}}\right| L_{p}\left(\ell_{\theta}\right)\right\|
$$

holds.

Proof. Step 1. We assume the unconditional convergence of $\sum_{\ell \in \mathbb{N}_{0}^{d}} f_{\ell}$ in $S_{p, \theta}^{r} F\left(\mathbb{T}^{d}\right.$ ) (or in case $\theta=\infty$ at least in $\left.S_{p, \nu}^{\tilde{\mathbf{r}}} F\left(\mathbb{T}^{d}\right)\right)$ and prove the inequality

$$
\left\|\sum_{\ell \in \mathbb{N}_{0}^{d}} f_{\ell}\left|S_{p, \theta}^{r} F\left(\mathbb{T}^{d}\right)\|\lesssim\| 2^{r \cdot j} f_{\boldsymbol{j}}\right| L_{p}\left(\ell_{\theta}\left(\mathbb{N}_{0}^{d}\right)\right)\right\| .
$$

We mimic Step 1 of the proof of [45, Theorem 3.4.1]. This is rather technical in the multivariate situation. For that reason we give a proof for the univariate situation first. Later we explain the necessary modifications for the multivariate situation. We prove

$$
\left\|f\left|F_{p, \theta}^{r}(\mathbb{T})\|\lesssim\| 2^{r j} f_{j}\right| L_{p}\left(\ell_{\theta}\left(\mathbb{N}_{0}\right)\right)\right\|
$$


by using methods from difference characterization of Triebel-Lizorkin spaces. We start by switching to the difference norm in $F_{p, \theta}^{r}(\mathbb{T})$ with $m>r$

$$
\left\|\sum_{\ell \in \mathbb{N}_{0}} f_{\ell} \mid F_{p, \theta}^{r}(\mathbb{T})\right\| \asymp\left\|\sum_{\ell \in \mathbb{N}_{0}} f_{\ell}\right\|_{p}+\left\|\left[\sum_{j=0}^{\infty} 2^{\theta j r}\left(2^{j} \int_{-2^{-j}}^{2^{-j}}\left|\Delta_{h}^{m}\left[\sum_{\ell \in \mathbb{N}_{0}} f_{\ell}\right]\right| d h\right)^{\theta}\right]^{\frac{1}{\theta}}\right\|_{p} .
$$

First we estimate the $L_{p}$-norm of $f$ and obtain trivially using either Hölder's inequality (in case $\theta \geq 1$ ) or the embedding $\ell_{\theta} \hookrightarrow \ell_{1}$ (in case $0<\theta<1$ ) the estimate

$$
\left\|\sum_{\ell \in \mathbb{N}_{0}} f_{\ell}\right\|_{p} \lesssim\left\|\left(\sum_{j \in \mathbb{N}_{0}} 2^{\theta r j}\left|f_{j}\right|^{\theta}\right)^{\frac{1}{\theta}}\right\|_{p}
$$

Let $a>0$ be a positive real number such that $a>\max \left\{\frac{1}{p}, \frac{1}{\theta}\right\}$ is fulfilled. Additionally choose in case $\min \{p, \theta\} \leq 1$

$$
0<\lambda<\min \{p, \theta\}
$$

such that

$$
r>(1-\lambda) a>\sigma_{p, \theta}
$$

This is possible since

$$
\begin{aligned}
(1-\lambda) a & >(1-\lambda) \max \left\{\frac{1}{p}, \frac{1}{\theta}\right\} \\
& \geq(1-\min \{p, \theta, 1\}) \max \left\{\frac{1}{p}, \frac{1}{\theta}\right\}=\sigma_{p, \theta} .
\end{aligned}
$$

In case $\min \{p, \theta\}>1$ we simply choose $\lambda=1$. Fix $j \in \mathbb{N}_{0}$ and use the identity

$$
\sum_{\ell \in \mathbb{N}_{0}} f_{\ell}=\sum_{\ell \in \mathbb{Z}} f_{j+\ell}
$$

with $f_{j+\ell}=0$ for $j+\ell<0$. The unconditional convergence of $\sum_{\ell \in \mathbb{Z}} f_{j+\ell}$ in $S_{p, \theta}^{r} F\left(\mathbb{T}^{d}\right)$ implies (by Lemma 3.5) an unconditional convergence also in $L_{1}\left(\mathbb{T}^{d}\right)$. Therefore we can estimate the integral means as follows

$$
2^{j} \int_{-2^{-j}}^{2^{-j}}\left|\Delta_{h}^{m}\left[\sum_{\ell \in \mathbb{N}_{0}} f_{j+\ell}\right]\right| d h \leq \sum_{\ell \in \mathbb{Z}} 2^{j} \int_{-2^{-j}}^{2^{-j}}\left|\Delta_{h}^{m} f_{j+\ell}(x)\right| d h .
$$

We split the sum over $\ell$

$$
\sum_{\ell \in \mathbb{Z}} 2^{j} \int_{-2^{-j}}^{2^{-j}}\left|\Delta_{h}^{m} f_{j+\ell}(x)\right| d h=\sum_{\ell \geq 0} 2^{j} \int_{-2^{-j}}^{2^{-j}}\left|\Delta_{h}^{m} f_{j+\ell}(x)\right| d h+\sum_{\ell<0} 2^{j} \int_{-2^{-j}}^{2^{-j}}\left|\Delta_{h}^{m} f_{j+\ell}(x)\right| d h
$$

and prove

$$
2^{j} \int_{-2^{-j}}^{2^{-j}}\left|\Delta_{h}^{m} f_{j+\ell}(x)\right| d h \lesssim \begin{cases}2^{\ell m} P_{2^{j+\ell}, a} f_{j+\ell} & : \quad \ell \geq 0 \\ 2^{(1-\lambda) \ell a}\left[P_{2^{j+\ell}, a} f_{j+\ell}\right]^{1-\lambda} M\left|f_{j+\ell}\right|^{\lambda} & : \quad \ell<0 .\end{cases}
$$

First we prove the case $\ell>0$. Applying Lemma A.6 immediately gives

$$
2^{j} \int_{-2^{-j}}^{2^{-j}}\left|\Delta_{h}^{m} f_{j+\ell}(x)\right| d h \lesssim 2^{\ell m} P_{2^{j+\ell}, a}(x) .
$$


In case $\ell<0$ with $\lambda<1$ we estimate as follows

$$
2^{j} \int_{-2^{-j}}^{2^{-j}}\left|\Delta_{h}^{m} f_{j+\ell}(x)\right| d h \lesssim 2^{j} \int_{-2^{-j}}^{2^{-j}}\left|\Delta_{h}^{m} f_{j+\ell}(x)\right|^{\lambda}\left|\Delta_{h}^{m} f_{j+\ell}(x)\right|^{1-\lambda} d h .
$$

Applying Lemma A.6 to the second factor yields

$$
\begin{aligned}
2^{j} \int_{-2^{-j}}^{2^{-j}}\left|\Delta_{h}^{m} f_{j+\ell}(x)\right| d h & \lesssim 2^{\ell(1-\lambda) a}\left[P_{2^{j+\ell}, a} f_{j+\ell}(x)\right]^{1-\lambda} 2^{j} \int_{-2^{-j}}^{2^{-j}}\left|\Delta_{h}^{m} f_{j+\ell}(x)\right|^{\lambda} d h \\
& \lesssim 2^{\ell(1-\lambda) a}\left[P_{2^{j+\ell}, a} f_{j+\ell}(x)\right]^{1-\lambda} M\left|f_{j+\ell}\right|^{\lambda}(x) .
\end{aligned}
$$

Attention in case $\min \{p, \theta\}>1$ with $\lambda=1$ the estimate in case $\ell<0$ simplifies to the HardyLittlewood maximal function of $\left|f_{j+\ell}\right|$. Inserting the decomposition in (4.6) together with the estimates obtained in (4.7) into the last term on the right hand side of (4.2) then we obtain by $\mu$-triangle inequality in $L_{p}\left(\ell_{\theta}(\mathbb{N})\right)$ with $\mu:=\min \{p, \theta, 1\}$

$$
\begin{aligned}
\| & {\left[\sum_{j=0}^{\infty} 2^{\theta j r}\left(2^{j} \int_{-2^{-j}}^{2^{-j}}\left|\Delta_{h}^{m}\left[\sum_{\ell \in \mathbb{N}_{0}} f_{j+\ell}\right]\right| d h\right)^{\theta}\right]^{\frac{1}{\theta}} \|_{p} } \\
\lesssim & {\left[\sum_{\ell \geq 0} 2^{\mu \ell(m-r)}\left\|2^{(j+\ell) r} P_{2^{j+\ell}, a} f_{j+\ell}(x) \mid L_{p}\left(\ell_{\theta}\left(\mathbb{N}_{0}\right)\right)\right\|^{\mu}+\sum_{\ell<0} 2^{\mu \ell[a(1-\lambda)-r]} \| 2^{(j+\ell) r}\left[P_{2^{j+\ell}, a} f_{2^{j+\ell}}(x)\right]^{1-\lambda}(x)\right.} \\
& \left.\times M\left|f_{j+\ell}\right|^{\lambda}(x) \mid L_{p}\left(\ell_{\theta}\left(\mathbb{N}_{0}\right)\right) \|^{\mu}\right]^{\frac{1}{\mu}} .
\end{aligned}
$$

To estimate the first summand we apply Theorem 4.5, which gives

$$
\left\|2^{(j+\ell) r} P_{2^{j+\ell}, a} f_{j+\ell}(x)\left|L_{p}\left(\ell_{\theta}\left(\mathbb{N}_{0}\right)\right)\|\lesssim\| 2^{(j+\ell) r} f_{j+\ell}(x)\right| L_{p}\left(\ell_{\theta}\left(\mathbb{N}_{0}\right)\right)\right\| .
$$

An index shift yields

$$
\left\|2^{(j+\ell) r} P_{2^{j+\ell}, a} f_{j+\ell}(x)\left|L_{p}\left(\ell_{\theta}\left(\mathbb{N}_{0}\right)\right)\|\lesssim\| 2^{j r} f_{j}(x)\right| L_{p}\left(\ell_{\theta}\left(\mathbb{N}_{0}\right)\right)\right\| .
$$

In case $\min \{p, \theta\} \leq 1$ with $\lambda<1$ we apply to the norm expression in (4.8) Hölder's inequality with $\frac{1}{1-\lambda}, \frac{1}{\lambda}$ twice and obtain

$$
\begin{aligned}
\left\|2^{(j+\ell) r}\left[P_{2^{j+\ell}, a} f_{j+\ell}(x)\right]^{1-\lambda} M\left|f_{j+\ell}\right|^{\lambda}(x) \mid L_{p}\left(\ell_{\theta}\left(\mathbb{N}_{0}\right)\right)\right\| \leq & \left\|2^{(j+\ell) r} P_{2^{j+\ell, a}} f_{j+\ell}(x) \mid L_{p}\left(\ell_{\theta}\left(\mathbb{N}_{0}\right)\right)\right\|^{1-\lambda} \\
& \times\left\|2^{(j+\ell) r}\left(M\left|f_{j+\ell}\right|^{\lambda}(x)\right)^{\frac{1}{\lambda}} \mid L_{p}\left(\ell_{\theta}\left(\mathbb{N}_{0}\right)\right)\right\|^{\lambda} .
\end{aligned}
$$

We skip this in case $\lambda=1$. Considering the factors in (4.10) separately we obtain by applying Theorem 4.5

$$
\left\|2^{(j+\ell) r} P_{2^{j+\ell}, a} f_{j+\ell}(x)\left|L_{p}\left(\ell_{\theta}\left(\mathbb{N}_{0}\right)\right)\|\lesssim\| 2^{(j+\ell) r} f_{j+\ell}(x)\right| L_{p}\left(\ell_{\theta}\left(\mathbb{N}_{0}\right)\right)\right\| .
$$

For the second factor we rewrite the $L_{p}\left(\ell_{\theta}\left(\mathbb{N}_{0}\right)\right)$-norm as a $L_{\frac{p}{\lambda}}\left(\ell_{\frac{\theta}{\lambda}}\left(\mathbb{N}_{0}\right)\right)$-norm. This allows for applying Theorem A.5.

$$
\begin{aligned}
\left\|2^{(j+\ell) r}\left(M\left|f_{j+\ell}\right|^{\lambda}(x)\right)^{\frac{1}{\lambda}} \mid L_{p}\left(\ell_{\theta}\left(\mathbb{N}_{0}\right)\right)\right\| & =\left\|2^{(j+\ell) r \lambda} M\left|f_{j+\ell}\right|^{\lambda}(x) \mid L_{\frac{p}{\lambda}}\left(\ell_{\frac{\theta}{\lambda}}\left(\mathbb{N}_{0}\right)\right)\right\|^{\frac{1}{\lambda}} \\
& \lesssim\left\|2^{(j+\ell) r \lambda}\left|f_{j+\ell}(x)\right|^{\lambda} \mid L_{\frac{p}{\lambda}}\left(\ell_{\frac{\theta}{\lambda}}\left(\mathbb{N}_{0}\right)\right)\right\|^{\frac{1}{\lambda}} \\
& =\left\|2^{(j+\ell) r} f_{j+\ell}(x) \mid L_{p}\left(\ell_{\theta}\left(\mathbb{N}_{0}\right)\right)\right\| .
\end{aligned}
$$


Inserting the estimates from (4.11) and (4.12) into (4.10) implies

$$
\left\|2^{(j+\ell) r}\left[P_{2^{j+\ell}, a} f_{j+\ell}(x)\right]^{1-\lambda} M\left|f_{j+\ell}\right|^{\lambda}(x)\left|L_{p}\left(\ell_{\theta}\left(\mathbb{N}_{0}\right)\right)\|\leq\| 2^{(j+\ell) r} f_{j+\ell}(x)\right| L_{p}\left(\ell_{\theta}\left(\mathbb{N}_{0}\right)\right)\right\| .
$$

A similar index shift as above yields

$$
\left\|2^{(j+\ell) r}\left[P_{2^{j+\ell, a}} f_{j+\ell}(x)\right]^{1-\lambda} M\left|f_{j+\ell}\right|^{\lambda}(x)\left|L_{p}\left(\ell_{\theta}\left(\mathbb{N}_{0}\right)\right)\|\leq\| 2^{j r} f_{j}(x)\right| L_{p}\left(\ell_{\theta}\left(\mathbb{N}_{0}\right)\right)\right\| .
$$

We continue estimating (4.8) and insert (4.9) and (4.13) to obtain

$\left\|\left[\sum_{j=0}^{\infty} 2^{\theta j r}\left(2^{j} \int_{-2^{-j}}^{2^{-j}}\left|\Delta_{h}^{m}\left[\sum_{\ell \in \mathbb{N}_{0}} f_{j+\ell}\right]\right| d h\right)^{\theta}\right]^{\frac{1}{\theta}}\right\|_{p} \lesssim\left\|2^{j r} f_{j} \mid L_{p}\left(\ell_{\theta}\left(\mathbb{N}_{0}\right)\right)\right\|\left[\sum_{\ell \geq 0} 2^{\mu \ell(m-r)}+\sum_{\ell<0} 2^{\mu \ell[a(1-\lambda)-r]]^{\frac{1}{\mu}}}\right.$.

Finally, the choice of the parameters $m, a, \lambda$ in (4.4) yields that the series in (4.14) converge to a constant. Altogether we obtain the desired bound

$$
\left\|\sum_{\ell \in \mathbb{N}_{0}} f_{\ell}\left|F_{p, \theta}^{r}(\mathbb{T})\|\lesssim\| 2^{j r} f_{j}\right| L_{p}\left(\ell_{\theta}\left(\mathbb{N}_{0}\right)\right)\right\|
$$

Step 2. We explain the modifications in the multivariate situation. This time we start computing the norm of $\sum_{\ell \in \mathbb{N}_{0}^{d}} f_{\ell} \in S_{p, \theta}^{r} F\left(\mathbb{T}^{d}\right)$ in terms of differences, cf. Theorem 3.9 ,

$$
\left\|\sum_{\ell \in \mathbb{N}_{0}^{d}} f_{\ell}\left|S_{p, \theta}^{r} F\left(\mathbb{T}^{d}\right)\left\|\asymp \sum_{e \subset[d]}\right\| f\right| S_{p, \theta}^{r} F\left(\mathbb{T}^{d}\right)\right\|_{e, m} .
$$

For each $e \subset[d]$ we have to show that

$$
\left\|\sum_{\boldsymbol{\ell} \in \mathbb{N}_{0}^{d}} f_{\ell}\left|S_{p, \theta}^{\boldsymbol{r}} F\left(\mathbb{T}^{d}\right)\left\|_{e, \boldsymbol{m}} \lesssim\right\| 2^{\boldsymbol{r j}} f_{\boldsymbol{j}}\right| L_{p}\left(\ell_{\theta}\left(\mathbb{N}_{0}^{d}\right)\right)\right\|
$$

holds. A full proof consists in applying the arguments from above to every single direction contained in $e$. Here the directionwise Hardy-Littlewood maximal function and corresponding maximal inequality come into play, see Definition 4.1 and Thms.4.2, 4.5. Since this requires an extensive case study in $e$ and $\boldsymbol{\ell}$ we refer to the proof given in detail in [45, Thm. 3.4.1, Step 1] where we have to replace the decomposition of $f$ used there by the representation $\sum_{\boldsymbol{\ell} \in \mathbb{Z}^{d}} f_{\boldsymbol{j}+\boldsymbol{\ell}}$. Step 3. We prove (i) in case $\theta<\infty$. To begin with, we define the set of sequences with finite index sets given by

$$
\mathfrak{E}:=\left\{\mathcal{E}=\left(\mathcal{E}_{n}\right)_{n \in \mathbb{N}}: \mathcal{E}_{n} \subset \mathbb{N}_{0}^{d},\left|\mathcal{E}_{n}\right|=n, \mathcal{E}_{n} \subset \mathcal{E}_{n+1} \text { for all } n \in \mathbb{N} \text {, and } \bigcup_{n=1}^{\infty} \mathcal{E}_{n}=\mathbb{N}_{0}^{d}\right\} .
$$

Every sequence in $\mathfrak{E}$ defines an order of summation. Furthermore for $\mathcal{E} \in \mathfrak{E}$ we define $F_{\mathcal{E}_{n}}:=$ $\sum_{\boldsymbol{j} \in \mathcal{E}_{n}} f_{\boldsymbol{j}}$. We take a second sequence $A \in \mathfrak{E}$ and consider $F_{\mathcal{E}_{n}}-F_{\mathcal{A}_{m}}$. This difference can be written as a sum with finitely many $f_{\boldsymbol{j}}$. This fulfills the assumptions necessary in Step 1 and yields

$$
\left\|F_{\mathcal{E}_{n}}-F_{\mathcal{A}_{m}} \mid S_{p, \theta}^{\boldsymbol{r}} F\left(\mathbb{T}^{d}\right)\right\| \lesssim\left\|\left(\sum_{\boldsymbol{j} \in\left(\mathcal{E}_{n} \cup \mathcal{A}_{m}\right) \backslash\left(\mathcal{E}_{n} \cap \mathcal{A}_{m}\right)} 2^{r \cdot \boldsymbol{j} \theta}\left|f_{\boldsymbol{j}}\right|^{\theta}\right)^{\frac{1}{\theta}}\right\|_{p}
$$

Obviously,

$$
\left(\sum_{\boldsymbol{j} \in\left(\mathcal{E}_{n} \cup \mathcal{A}_{m}\right) \backslash\left(\mathcal{E}_{n} \cap \mathcal{A}_{m}\right)} 2^{\boldsymbol{r} \cdot \boldsymbol{j} \theta}\left|f_{\boldsymbol{j}}\right|^{\theta}\right)^{\frac{1}{\theta}} \leq\left(\sum_{\boldsymbol{j} \in \mathbb{N}_{0}^{d}} 2^{r \cdot \boldsymbol{j} \theta}\left|f_{\boldsymbol{j}}\right|^{\theta}\right)^{\frac{1}{\theta}} \in L_{p}\left(\mathbb{T}^{d}\right)
$$


holds almost everywhere. Therefore Lebesgue's dominated convergence theorem yields that we find for every $\varepsilon>0$ a $n_{0} \in \mathbb{N}$ such that

$$
\left\|F_{\mathcal{E}_{n}}-F_{\mathcal{A}_{m}} \mid S_{p, \theta}^{r} F\left(\mathbb{T}^{d}\right)\right\| \leq \varepsilon
$$

holds for all $m, n>n_{0}$. Finally this implies unconditional convergence in $S_{p, \theta}^{r} F\left(\mathbb{T}^{d}\right)$. In case $\theta=\infty$ we stress on the embeddings

$$
S_{p, 1}^{s} F\left(\mathbb{T}^{d}\right) \hookrightarrow S_{p, \nu}^{\tilde{r}} F\left(\mathbb{T}^{d}\right)
$$

and

$$
\left\|2^{s \cdot j} f_{j}\left|L_{p}\left(\ell_{1}\right)\|\lesssim\| 2^{r \cdot j} f_{j}\right| L_{p}\left(\ell_{\infty}\right)\right\|,
$$

where $\boldsymbol{r}>\boldsymbol{s}>\sigma_{p, \nu}, \boldsymbol{s}>\tilde{\boldsymbol{r}}$ and $0<\nu \leq \infty$. Applying the arguments from above to $S_{p, 1}^{\boldsymbol{s}} F\left(\mathbb{T}^{d}\right)$ yields the result for $S_{p, \nu}^{\tilde{r}} F\left(\mathbb{T}^{d}\right)$.

We will also need the following diagonal embedding relation which is the periodic counterpart of [32, Prop. 2.4.1], see also the diagonal embedding in Lemma 3.5, (vi) above.

Lemma 4.7. Let $0<p<q<\infty$ and $0<\theta, \nu \leq \infty$. Then

$$
\left\|\left(\sum_{\boldsymbol{j} \in \mathbb{N}_{0}^{d}}\left|f_{\boldsymbol{j}}\right|^{\nu}\right)^{\frac{1}{\nu}}\right\|_{q} \lesssim\left\|\left(\sum_{\boldsymbol{j} \in \mathbb{N}_{0}^{d}} 2^{\theta|\boldsymbol{j}|_{1}(1 / p-1 / q)}\left|f_{\boldsymbol{j}}\right|^{\theta}\right)^{\frac{1}{\theta}}\right\|_{p}
$$

holds for all $\left(f_{\boldsymbol{j}}\right)_{\boldsymbol{j} \in \mathbb{N}_{0}^{d}}$ such that $f_{\boldsymbol{j}} \in \mathcal{T}_{\boldsymbol{j}}^{0}$.

Let us finally state the counterpart of Theorem 4.6 for the $B$-case.

Theorem 4.8. Let $0<p \leq \infty, 0<\theta \leq \infty, \boldsymbol{r} \in \mathbb{R}^{d}$ with $\boldsymbol{r}>\sigma_{p}$ and $\left(f_{\boldsymbol{j}}\right)_{\boldsymbol{j} \in \mathbb{N}_{0}^{d}}$ such that $f_{\boldsymbol{j}} \in \mathcal{T}_{\boldsymbol{j}}^{0}$ and $\left\|2^{r \cdot \boldsymbol{j}} f_{\boldsymbol{j}} \mid \ell_{\theta}\left(L_{p}\right)\right\|<\infty$. Then

(i) $\sum_{j \in \mathbb{N}_{0}^{d}} f_{j}$ converges unconditionally in $S_{p, \theta}^{r} B\left(\mathbb{T}^{d}\right)$ if $\max \{p, \theta\}<\infty$ and in every $S_{p, \nu}^{\tilde{\mathbf{r}}} B\left(\mathbb{T}^{d}\right)$ with $0<\nu \leq \infty$ and $\tilde{\boldsymbol{r}}<\boldsymbol{r}$.

(ii) it holds

$$
\left\|\sum _ { \boldsymbol { j } \in \mathbb { N } _ { 0 } ^ { d } } f _ { \boldsymbol { j } } | S _ { p , \theta } ^ { r } B ( \mathbb { T } ^ { d } ) \| \lesssim \| 2 ^ { r \cdot j } f _ { \boldsymbol { j } } | \ell _ { \theta } \left(L_{p}\left(\mathbb{T}^{d}\right) \|\right.\right.
$$

Proof. We follow the proof of Theorem 4.6 line by line and point out the necessary modifications for the $B$-case. To convince the reader we explain this modifications for (ii) in the univariate case. Again, we prove

$$
\left\|\sum_{\ell \in \mathbb{N}_{0}} f_{\ell}\left|B_{p, \theta}^{r}(\mathbb{T})\|\lesssim\| 2^{r j} f_{j}\right| L_{p}\left(\ell_{\theta}\left(\mathbb{N}_{0}\right)\right)\right\|
$$

by using methods from difference characterization. We start by switching to the difference norm in $B_{p, \theta}^{r}(\mathbb{T})$ with $m>r$

$$
\left\|\sum_{\ell \in \mathbb{N}_{0}} f_{\ell} \mid B_{p, \theta}^{r}(\mathbb{T})\right\| \asymp\left\|\sum_{\ell \in \mathbb{N}_{0}} f_{\ell}\right\|_{p}+\left(\sum_{j=0}^{\infty} 2^{\theta j r}\left\|2^{j} \int_{-2^{-j}}^{2^{-j}}\left|\Delta_{h}^{m}\left[\sum_{\ell \in \mathbb{N}_{0}} f_{\ell}\right]\right| d h\right\|_{p}^{\theta}\right)^{\frac{1}{\theta}} .
$$


First we estimate the $L_{p}$-norm of $f$ and obtain trivially using either Hölder's inequality (in case $\min \{p, \theta\}>1$ or $p<\min \{1, \theta\})$ or the embedding $\ell_{\theta} \hookrightarrow \ell_{1}$ (otherwise) the estimate

$$
\left\|\sum_{\ell \in \mathbb{N}_{0}} f_{\ell}\right\|_{p} \lesssim\left(\sum_{j \in \mathbb{N}_{0}} 2^{\theta r j}\left\|f_{j}\right\|_{p}^{\theta}\right)^{\frac{1}{\theta}}
$$

Let $a>0$ be a positive real number such that $a>\frac{1}{p}$ is fulfilled. Additionally, in case $p>1$ we choose $\lambda=1$. Whereas in case $p \leq 1$ we choose

$$
0<\lambda<p
$$

such that

$$
r>(1-\lambda) a>(1-\lambda) \frac{1}{p} \geq(1-p) \frac{1}{p}=\sigma_{p} .
$$

For the second term in (4.16) the estimates in (4.5), (4.6) and (4.7) yield

$$
\begin{aligned}
& \left(\sum_{j=0}^{\infty} 2^{\theta j r}\left\|2^{j} \int_{-2^{-j}}^{2^{-j}}\left|\Delta_{h}^{m}\left[\sum_{\ell \in \mathbb{Z}^{d}} f_{j+\ell}\right]\right| d h\right\|_{p}^{\theta}\right)^{\frac{1}{\theta}} \\
& \lesssim\left[\sum_{\ell \geq 0} 2^{\mu \ell(m-r)}\left\|2^{(j+\ell) r} P_{2^{j+\ell}, a} f_{j+\ell}(x) \mid \ell_{\theta}\left(L_{p}(\mathbb{T})\right)\right\|^{\mu}\right. \\
& \left.\quad+\sum_{\ell<0} 2^{\mu \ell[a(1-\lambda)-r]}\left\|2^{(j+\ell) r}\left[P_{2^{j+\ell, a}} f_{2^{j+\ell}}(x)\right]^{1-\lambda} \times M\left|f_{j+\ell}\right|^{\lambda}(x) \mid \ell_{\theta}\left(L_{p}(\mathbb{T})\right)\right\|^{\mu}\right]^{\frac{1}{\mu}}
\end{aligned}
$$

with $\mu=\min \{p, \theta, 1\}$. The $L_{p}(\mathbb{T})$-norm is now the inner norm in the sequence spaces. For that reason it suffices to use simpler (non-vector valued) maximal inequalities. We apply Theorem 4.4 to the first summand, which gives

$$
\left\|2^{(j+\ell) r} P_{2^{j+\ell}, a} f_{j+\ell}(x)\left|\ell_{\theta}\left(L_{p}(\mathbb{T})\right)\|\lesssim\| 2^{(j+\ell) r} f_{j+\ell}(x)\right| \ell_{\theta}\left(L_{p}(\mathbb{T})\right)\right\| .
$$

An index shift yields

$$
\left\|2^{(j+\ell) r} P_{2^{j+\ell, a}} f_{j+\ell}(x)\left|\ell_{\theta}\left(L_{p}(\mathbb{T})\right)\|\lesssim\| 2^{j r} f_{j}(x)\right| \ell_{\theta}\left(L_{p}(\mathbb{T})\right)\right\| .
$$

In case $p \leq 1$ we apply Hölder's inequality to the second summand in (4.17) with $\frac{1}{1-\lambda}$, $\frac{1}{\lambda}$ and obtain

$$
\begin{aligned}
\left\|2^{(j+\ell) r}\left[P_{2^{j+\ell}, a} f_{j+\ell}(x)\right]^{1-\lambda} M\left|f_{j+\ell}\right|^{\lambda}(x) \mid \ell_{\theta}\left(L_{p}(\mathbb{T})\right)\right\|^{\theta} \leq & \left(\sum_{j \in \mathbb{N}_{0}} 2^{\theta(j+\ell) r}\left\|P_{2^{j+\ell}, a} f_{j+\ell}(x) \mid L_{p}(\mathbb{T})\right\|^{(1-\lambda) \theta}\right. \\
& \left.\times\left\|\left(M\left|f_{j+\ell}\right|^{\lambda}(x)\right)^{\frac{1}{\lambda}} \mid L_{p}(\mathbb{T})\right\|^{\lambda \theta}\right)^{\frac{1}{\theta}} .
\end{aligned}
$$

This can be skipped in case $p>1$. Applying the maximal inequalities stated in Theorem 4.4 and Theorem A.4 (together with a trick similar to (4.12) ) yields

$$
\begin{aligned}
\left\|2^{(j+\ell) r}\left[P_{2^{j+\ell}, a} f_{j+\ell}(x)\right]^{1-\lambda} M\left|f_{j+\ell}\right|^{\lambda}(x) \mid \ell_{\theta}\left(L_{p}(\mathbb{T})\right)\right\| & \lesssim\left(\sum_{j \in \mathbb{N}_{0}} 2^{\theta(j+\ell) r}\left\|f_{j+\ell}(x) \mid L_{p}(\mathbb{T})\right\|^{\theta}\right)^{\frac{1}{\theta}} \\
& \lesssim\left\|2^{j r} f_{j}(x) \mid \ell_{\theta}\left(L_{p}(\mathbb{T})\right)\right\| .
\end{aligned}
$$


Hence, the estimates from (4.18) and (4.19) imply

$$
\begin{aligned}
& \left(\sum_{j=0}^{\infty} 2^{\theta j r}\left\|2^{j} \int_{-2^{-j}}^{2^{-j}}\left|\Delta_{h}^{m}\left[\sum_{\ell \in \mathbb{Z}^{d}} f_{j+\ell}\right]\right| d h\right\|_{p}^{\theta}\right)^{\frac{1}{\theta}} \\
& \lesssim\left[\sum_{\ell \geq 0} 2^{\mu \ell(m-r)}+\sum_{\ell<0} 2^{\mu \ell[a(1-\lambda)-r]}\right]^{\frac{1}{\mu}}\left\|2^{j r} f_{j}(x) \mid \ell_{\theta}\left(L_{p}(\mathbb{T})\right)\right\| .
\end{aligned}
$$

The choice of $\lambda, a$ and $m$ relatively to $r$ ensures the convergence of the series to an absolute constant. This concludes the proof in the univariate case. For the multivariate situation see the comments in Step 2 of Theorem 4.6.

\section{$5 \quad$ Sampling representations}

In this section we provide theorems that allow for replacing the Fourier analytic building blocks $\delta_{\boldsymbol{j}}[f]$ used to define the spaces $S_{p, \theta}^{\boldsymbol{r}} F\left(\mathbb{T}^{d}\right)$ and $S_{p, \theta}^{\boldsymbol{r}} B\left(\mathbb{T}^{d}\right)$ by building blocks $q_{\boldsymbol{j}}^{L}[f]$, cf. Definition 3.3. Here we will prove the following main results.

Theorem 5.1. Let $0<p<\infty, 0<\theta \leq \infty, L>\max \left\{\frac{1}{p}, \frac{1}{\theta}\right\}(L=1$ requires $\theta<\infty)$ and $\boldsymbol{r}>\max \left\{\frac{1}{p}, \frac{1}{\theta}\right\}$ then the (quasi-)norms

$$
\left\|f\left|S_{p, \theta}^{r} F\left(\mathbb{T}^{d}\right)\|\asymp\| 2^{r \cdot j} q_{j}^{L}(f)\right| L_{p}\left(\ell_{\theta}\right)\right\|
$$

are equivalent for all $f \in S_{p, \theta}^{r} F\left(\mathbb{T}^{d}\right)$.

Proof. The result is a consequence of Theorem 5.7 together with Theorem 4.6. For the case $L=1$ we refer to Theorem 5.13 ,

Remark 5.2. We strongly conjecture the optimality of the condition on $L$ in the above theorems, see also Remark 3.4. (ii) above.

For the $B$-case weaker conditions on $\boldsymbol{r}$ and $L$ are sufficient.

Theorem 5.3. Let $0<p, \theta \leq \infty, L>\frac{1}{p}(L=1$ requires $p<\infty)$ and $\boldsymbol{r}>\frac{1}{p}$ then the (quasi-)norms

$$
\left\|f\left|S_{p, \theta}^{r} B\left(\mathbb{T}^{d}\right)\|\asymp\| 2^{r \cdot j} q_{j}^{L}(f)\right| \ell_{\theta}\left(L_{p}\right)\right\|
$$

are equivalent for all $f \in S_{p, \theta}^{\boldsymbol{r}} B\left(\mathbb{T}^{d}\right)$.

Proof. The proof is a consequence of Theorem 4.8 together with Theorem 5.9, For the case $L=1$ we refer to Theorem 5.14 ,

Remark 5.4. In case of $S_{p, \theta}^{\boldsymbol{r}} B\left(\mathbb{T}^{d}\right)$ with $p \geq 1$ and $\boldsymbol{r}>1 / p$ similar characterizations were proved by Dinh Dũng [5, 6, [7] using the following variant of the de la Vallée-Poussin kernel

$$
V_{j}(x)=\frac{\sin \left(2^{j-1} x\right) \sin \left(32^{j-1} x\right)}{2^{2 j} 3 \sin ^{2}\left(\frac{x}{2}\right)},
$$

which yields to an interpolation operator on $3 \cdot 2^{j}$ equidistant nodes. We can reproduce and extend this result to the Triebel-Lizorkin scale as well as to $p>1 / 2$ with straight-forward modifications of the arguments used in Theorems 5.9 below. Note, that our proof only uses a reproduction and a decay property of the kernel. Also the de la Vallée Poussin sampling operator $R_{m}$ used by Temlyakov in [42, I.6] is admissible here. 


\subsection{The case of quadratically decaying kernels with $L \geq 2$}

Let us first deal with kernels providing at least a quadratic decay according to Lemma 2.6. We introduce the characteristic function $\chi_{j, u}$ of the dyadic interval $\left[2 \pi u / 2^{j}, 2 \pi(u+1) / 2^{j}\right]$ indexed by $j \in \mathbb{N}_{0}$ and $u \in \mathbb{Z}$. For $\boldsymbol{j} \in \mathbb{N}_{0}^{d}$ and $\boldsymbol{u} \in \mathbb{Z}^{d}$ we denote with

$$
\chi_{\boldsymbol{j}, \boldsymbol{u}}(\boldsymbol{x}):=\prod_{i=1}^{d} \chi_{j_{i}, u_{i}}\left(x_{i}\right)
$$

the characteristic function of the respective parallelepiped. The following lemma represents the "hyperbolic" version of [19, Lem. 3, 7]. The lemma is originally due to Kyriazis [21, Lem. 7.1]. For the convenience of the reader we will give a proof.

Lemma 5.5. Let $0<\lambda \leq 1$ and $L>\frac{1}{\lambda}$. For any sequence $\left(\lambda_{\boldsymbol{u}}\right)_{\boldsymbol{u} \in A_{\boldsymbol{j}}(\boldsymbol{x})}$ of complex numbers and every $\boldsymbol{j} \in \mathbb{N}_{0}^{d}$ we have

$$
\sum_{\boldsymbol{u} \in \mathbb{Z}^{d}}\left|\lambda_{\boldsymbol{j}, \boldsymbol{u}}\right| \prod_{i=1}^{d}\left(1+2^{j}\left|x_{i}-x_{u_{i}}^{j_{i}}\right|\right)^{-L} \leq C\left[M\left|\sum_{\boldsymbol{u} \in \mathbb{Z}^{d}} \lambda_{\boldsymbol{j}, \boldsymbol{u}} \chi_{\boldsymbol{j}, \boldsymbol{u}}\right|^{\lambda}(\boldsymbol{x})\right]^{\frac{1}{\lambda}}
$$

with a constant $C$ independent of $\boldsymbol{j},\left(\lambda_{\boldsymbol{j}, \boldsymbol{u}}\right)_{\boldsymbol{u}}$ and $\boldsymbol{x}$.

Proof. We follow the proof given in [19, Lem. 7]. Let $\delta=L-1 / \lambda>0$. We define the following decomposition of $\mathbb{Z}^{d}$. Let $\boldsymbol{x} \in \mathbb{R}^{d}$ then

$$
\Omega_{\boldsymbol{k}}(\boldsymbol{x}):=\Omega_{k_{1}} \cdot \ldots \cdot \Omega_{k_{d}}, \quad \boldsymbol{k} \in \mathbb{N}_{0}^{d},
$$

with

$$
\begin{aligned}
\Omega_{k_{i}} & :=\left\{u_{i} \in A_{j_{i}}\left(x_{i}\right): \pi 2^{k_{i}-1}<2^{j_{i}}\left|x_{i}-x_{u_{i}}^{j_{i}}\right| \leq \pi 2^{k_{i}}\right\} \\
\Omega_{0} & :=\left\{u_{i} \in A_{j_{i}}\left(x_{i}\right): 2^{j_{i}}\left|x_{i}-x_{u_{i}}^{j_{i}}\right| \leq \pi\right\}
\end{aligned}
$$

We estimate

$$
\begin{aligned}
\sum_{\boldsymbol{u} \in \mathbb{Z}^{d}} & \left|\lambda_{\boldsymbol{j}, \boldsymbol{u}}\right| \prod_{i=1}^{d}\left(1+2^{j_{i}}\left|x_{i}-x_{u_{i}}^{j_{i}}\right|\right)^{-L} \\
& =\sum_{\boldsymbol{k} \in \mathbb{N}_{0}^{d}} \sum_{\boldsymbol{u} \in \Omega_{\boldsymbol{k}}(\boldsymbol{x})}\left|\lambda_{\boldsymbol{j}, \boldsymbol{u}}\right| \prod_{i=1}^{d}\left(1+2^{j_{i}}\left|x_{i}-x_{u_{i}}^{j_{i}}\right|\right)^{-L} \\
& \lesssim \sum_{\boldsymbol{k} \in \mathbb{N}_{0}^{d}} \sum_{\boldsymbol{u} \in \Omega_{\boldsymbol{k}}(\boldsymbol{x})}\left|\lambda_{\boldsymbol{j}, \boldsymbol{u}}\right| 2^{-\delta|\boldsymbol{k}|_{1}-|\boldsymbol{k}|_{1} / \lambda} \lesssim \sup _{\substack{k_{i} \leq j_{i} \\
i \in[d]}}\left(\sum_{\boldsymbol{u} \in \Omega_{\boldsymbol{k}}(\boldsymbol{x})}\left|\lambda_{\boldsymbol{j}, \boldsymbol{u}}\right|\right) 2^{-|\boldsymbol{k}|_{1} / \lambda} \\
& \lesssim\left(\sup _{\boldsymbol{k} \in \mathbb{N}_{0}^{d}} 2^{-|\boldsymbol{k}|_{1}} \sum_{\boldsymbol{u} \in \Omega_{\boldsymbol{k}}(\boldsymbol{x})}\left|\lambda_{\boldsymbol{j}, \boldsymbol{u}}\right|^{\lambda}\right)^{1 / \lambda} .
\end{aligned}
$$

Note, that

$$
\int_{\boldsymbol{u} \in \Omega_{\boldsymbol{k}}(\boldsymbol{x})} \sum_{Q_{\boldsymbol{j}, \boldsymbol{u}}}\left|\lambda_{\boldsymbol{j}, \boldsymbol{\omega}}\right|^{\lambda} \chi_{\boldsymbol{j}, \boldsymbol{\omega}}(\boldsymbol{y}) d \boldsymbol{y}=2^{-|\boldsymbol{j}|_{1}} \sum_{\boldsymbol{u} \in \Omega_{\boldsymbol{k}}(\boldsymbol{x})}\left|\lambda_{\boldsymbol{j}, \boldsymbol{u}}\right|^{\lambda}
$$


and hence

$$
2^{-|\boldsymbol{k}|_{1}} \sum_{\boldsymbol{u} \in \Omega_{\boldsymbol{k}}(\boldsymbol{x})}\left|\lambda_{\boldsymbol{j}, \boldsymbol{u}}\right|^{\lambda}=2^{-|\boldsymbol{k}|_{1}+|\boldsymbol{j}|_{1}} \int_{\boldsymbol{u} \in \Omega_{\boldsymbol{k}}(\boldsymbol{x})} \sum_{Q_{\boldsymbol{j}, \boldsymbol{u}}}\left|\lambda_{\boldsymbol{\omega} \in \Omega_{\boldsymbol{k}}(\boldsymbol{x})}\right|^{\lambda} \chi_{\boldsymbol{j}, \boldsymbol{\omega}}(\boldsymbol{y}) d \boldsymbol{y} .
$$

We further observe that for $Q(\boldsymbol{x}):=\bigcup_{\boldsymbol{u} \in \Omega_{\boldsymbol{k}}(\boldsymbol{x})} Q_{\boldsymbol{j}, \boldsymbol{u}}$ we have

$$
|Q(\boldsymbol{x})| \asymp 2^{|\boldsymbol{k}|_{1}-|\boldsymbol{j}|_{1}} .
$$

and $\boldsymbol{x} \in Q(\boldsymbol{x})$. Recalling the definition of the Hardy-Littlewood maximal functions in Definition A.3 we obtain

$$
\begin{aligned}
\int_{Q(\boldsymbol{x})} \sum_{\boldsymbol{u} \in \Omega_{\boldsymbol{k}}(\boldsymbol{x})}\left|\lambda_{\boldsymbol{j}, \boldsymbol{u}}\right|^{\lambda} \chi_{\boldsymbol{j}, \boldsymbol{u}}(\boldsymbol{y}) d \boldsymbol{y} & \lesssim 2^{|\boldsymbol{k}|_{1}-|\boldsymbol{j}|_{1}} \frac{1}{|Q(\boldsymbol{x})|} \int_{Q(\boldsymbol{x})} \sum_{\boldsymbol{u} \in \Omega_{\boldsymbol{k}}(\boldsymbol{x})}\left|\lambda_{\boldsymbol{j}, \boldsymbol{u}}\right|^{\lambda} \chi_{\boldsymbol{j}, \boldsymbol{u}}(\boldsymbol{y}) d \boldsymbol{y} \\
& \leq 2^{|\boldsymbol{k}|_{1}-|\boldsymbol{j}|_{1}} M\left|\sum_{\boldsymbol{u} \in \mathbb{Z}^{d}} \lambda_{\boldsymbol{j}, \boldsymbol{u}} \chi_{\boldsymbol{j}, \boldsymbol{u}}\right|^{\lambda}(\boldsymbol{x}) .
\end{aligned}
$$

Putting this into (5.4) we obtain

$$
2^{-|\boldsymbol{k}|_{1}} \sum_{\boldsymbol{u} \in \Omega_{\boldsymbol{k}}(\boldsymbol{x})}\left|\lambda_{\boldsymbol{j}, \boldsymbol{u}}\right|^{\lambda} \lesssim M\left|\sum_{\boldsymbol{u} \in \mathbb{Z}^{d}} \lambda_{\boldsymbol{j}, \boldsymbol{u}} \chi_{\boldsymbol{j}, \boldsymbol{u}}\right|^{\lambda}(\boldsymbol{x}) .
$$

Finally, we plug this estimate into (5.3) and obtain the desired result.

Proposition 5.6. Let $\boldsymbol{\ell}, \boldsymbol{j} \in \mathbb{N}_{0}^{d}, 0<\lambda \leq 1, L \in \mathbb{N}$ with $L>\frac{1}{\lambda}$ and $a>0$. Let further $f \in C\left(\mathbb{T}^{d}\right)$.

(i) Then

$$
\left|I_{j}^{L}[f](\boldsymbol{x})\right| \lesssim 2^{a|\ell|_{1}}\left[M\left|P_{\mathbf{2}^{j+\ell}, a} f\right|^{\lambda}(\boldsymbol{x})\right]^{\frac{1}{\lambda}}
$$

(ii) and also

$$
\left|q_{j}^{L}[f](\boldsymbol{x})\right| \lesssim 2^{a|\ell|_{1}}\left[M\left|P_{2^{j+\ell}, a} f\right|^{\lambda}(\boldsymbol{x})\right]^{\frac{1}{\lambda}}
$$

holds with a constant independent of $\boldsymbol{\ell}, \boldsymbol{j}, \boldsymbol{x}$ and $f$.

Proof. We start proving (i). Recall the notation from (2.10). Periodicity of $f$ and $K_{\pi, j}^{L}$ yields

$$
\begin{aligned}
I_{\boldsymbol{j}}^{L} f(\boldsymbol{x}) \mid & =\sum_{\boldsymbol{u} \in A_{j}} f\left(\boldsymbol{x}_{\boldsymbol{u}}^{\boldsymbol{j}}\right) K_{\pi^{d}, \boldsymbol{j}}^{L}\left(\boldsymbol{x}-\boldsymbol{x}_{\boldsymbol{u}}^{\boldsymbol{j}}\right) \\
& =\sum_{\boldsymbol{u} \in A_{\boldsymbol{j}}(\boldsymbol{x})} f\left(\boldsymbol{x}_{\boldsymbol{u}}^{\boldsymbol{j}}\right) K_{\pi^{d}, \boldsymbol{j}}^{L}\left(\boldsymbol{x}-\boldsymbol{x}_{\boldsymbol{u}}^{\boldsymbol{j}}\right)
\end{aligned}
$$

Lemma 2.6 with $\left|x_{i}-x_{u_{i}}^{j_{i}}\right| \leq \pi$ gives

$$
\begin{aligned}
\left|I_{\boldsymbol{j}}^{L} f(\boldsymbol{x})\right| & \leq \sum_{\boldsymbol{u} \in A_{\boldsymbol{j}}(\boldsymbol{x})}\left|f\left(\boldsymbol{x}_{\boldsymbol{u}}^{\boldsymbol{j}}\right) \prod_{i=1}^{d} K_{\pi, j_{i}}^{L}\left(x_{i}-x_{u_{i}}^{j_{i}}\right)\right| \\
& \lesssim \sum_{\boldsymbol{u} \in \mathbb{Z}^{d}}\left|\lambda_{\boldsymbol{j}, \boldsymbol{u}}\right| \prod_{i=1}^{d}\left(1+2^{j_{i}}\left|x_{i}-x_{u_{i}}^{j_{i}}\right|\right)^{-L}
\end{aligned}
$$


where we used the notation

$$
\lambda_{\boldsymbol{j}, \boldsymbol{u}}:=\sup _{\substack{\boldsymbol{y}:\left|y_{i}-x_{u_{i}}^{j_{i}}\right|<\frac{2 \pi}{2^{j_{i}}} \\ i \in[d]}}|f(\boldsymbol{y})|
$$

Applying Lemma 5.5 gives

$$
\left|I_{\boldsymbol{j}}^{L} f(\boldsymbol{x})\right| \lesssim\left[M\left|\sum_{\boldsymbol{u} \in \mathbb{Z}^{d}} \lambda_{\boldsymbol{j}, \boldsymbol{u}} \chi_{\boldsymbol{j}, \boldsymbol{k}}\right|^{\lambda}(\boldsymbol{x})\right]^{\frac{1}{\lambda}} .
$$

Taking $\boldsymbol{z} \in \operatorname{supp} \chi_{\boldsymbol{j}, \boldsymbol{u}^{*}}$ gives for any $a>0$

$$
\begin{aligned}
\left|\sum_{\boldsymbol{u} \in \mathbb{Z}^{d}} \lambda_{\boldsymbol{j}, \boldsymbol{u}} \chi_{\boldsymbol{j}, \boldsymbol{u}}(\boldsymbol{z})\right|=\left|\lambda_{\boldsymbol{j}, \boldsymbol{u}^{*}}\right| & =\sup _{\substack{\boldsymbol{y}:\left|y_{i}-x_{u_{i}^{*}}^{j_{i}}\right|<\frac{2 \pi}{2^{j_{i}}} \\
i \in[d]}}|f(\boldsymbol{y})| \\
& \lesssim \sup _{\substack{\boldsymbol{y}:\left|y_{i}-z_{i}\right|<\frac{4 \pi}{2^{j_{i}}} \\
i \in[d]}} \frac{|f(\boldsymbol{y})|}{\prod_{i=1}^{d}\left(1+2^{j_{i}}\left|y_{i}-z_{i}\right|\right)^{a}} .
\end{aligned}
$$

Finally, Lemma A.7 yields

$$
\left|\sum_{\boldsymbol{u} \in \mathbb{Z}^{d}} \lambda_{\boldsymbol{j}, \boldsymbol{u}} \chi_{\boldsymbol{j}, \boldsymbol{u}}(\boldsymbol{z})\right| \lesssim 2^{|\ell|{ }_{1} a} P_{\mathbf{2}^{j}, a} f(\boldsymbol{z})
$$

Inserting (5.6) into (5.5) finishes the proof of (i). The bound in (ii) is a trivial consequence of applying triangle inequality to (2.9) and (i)

$$
\left|q_{\boldsymbol{j}}^{L}[f](\boldsymbol{x})\right| \leq \sum_{\boldsymbol{b} \in\{-1,0\}^{d}}\left|I_{\boldsymbol{j}+\boldsymbol{b}}^{L}[f](\boldsymbol{x})\right| .
$$

Theorem 5.7. Let $0<p, \theta \leq \infty(p<\infty), L>\max \left\{\frac{1}{p}, \frac{1}{\theta}, 1\right\}$ and $\boldsymbol{r}>\max \left\{\frac{1}{p}, \frac{1}{\theta}\right\}$.

(i) Then every $f \in S_{p, \theta}^{r} F\left(\mathbb{T}^{d}\right)$ admits the representation

$$
f=\sum_{j \in \mathbb{N}_{0}^{d}} q_{j}^{L}[f]
$$

with unconditional convergence in $S_{p, \theta}^{r} F\left(\mathbb{T}^{d}\right)$ in case $0<\theta<\infty$ and with unconditional convergence in $S_{p, \nu}^{\tilde{\boldsymbol{r}}} F\left(\mathbb{T}^{d}\right)$ for every $\boldsymbol{r}>\tilde{\boldsymbol{r}}$ and $0<\nu \leq \infty$ in case $\theta=\infty$.

(ii) There is a constant $C>0$ independent of $f$ such that

$$
\left\|2^{r \cdot j} q_{\boldsymbol{j}}^{L}(f)\left|L_{p}\left(\ell_{\theta}\right)\|\leq C\| f\right| S_{p, \theta}^{r} F\left(\mathbb{T}^{d}\right)\right\|
$$

holds for all $f \in S_{p, \theta}^{r} F\left(\mathbb{T}^{d}\right)$. 
Proof. Step 1. We prove (5.9). To begin with we choose $a>0$ such that $\boldsymbol{r}>a>\max \left\{\frac{1}{p}, \frac{1}{q}\right\}$ is fulfilled. Let $f \in S_{p, \theta}^{r} F\left(\mathbb{T}^{d}\right)$. We start for $j \in \mathbb{N}_{0}^{d}$ with the Fourier decomposition

$$
f(\boldsymbol{x})=\sum_{\ell \in \mathbb{Z}^{d}} \delta_{\boldsymbol{j}+\ell}[f](\boldsymbol{x}),
$$

cf. (3.1), where $\delta_{\boldsymbol{j}}[f]:=0$ for $\boldsymbol{j} \in \mathbb{Z}^{d} \backslash \mathbb{N}_{0}^{d}$. This series converges unconditionally in $C\left(\mathbb{T}^{d}\right)$, due to the embedding $S_{p, \theta}^{r} F\left(\mathbb{T}^{d}\right) \hookrightarrow \mathbb{C}\left(\mathbb{T}^{d}\right)$. That yields the point-wise estimate

$$
\left|q_{\boldsymbol{j}}^{L}[f](\boldsymbol{x})\right| \leq \sum_{\boldsymbol{\ell} \in \mathbb{Z}^{d}}\left|q_{\boldsymbol{j}}^{L}\left[\delta_{\boldsymbol{j}+\ell}[f]\right](\boldsymbol{x})\right| .
$$

For the sake of simplicity we assume that the constants $A, B, C$ in Definition 3.2 are chosen in such a way that $\delta_{j}[f] \in \mathcal{T}_{j}^{L}$ is fulfilled for all $j \in \mathbb{N}_{0}^{d}$. Then Proposition 2.10 implies

$$
\left|q_{\boldsymbol{j}}^{L}[f](\boldsymbol{x})\right| \leq \sum_{\ell \geq 0}\left|q_{\boldsymbol{j}}^{L}\left[\delta_{\boldsymbol{j}+\boldsymbol{\ell}}[f]\right](\boldsymbol{x})\right| .
$$

Applying Proposition 5.6, (ii) we obtain

$$
\left|q_{\boldsymbol{j}}^{L}[f](\boldsymbol{x})\right| \lesssim \sum_{\boldsymbol{\ell} \geq 0} 2^{a|\boldsymbol{\ell}|_{1}}\left[M\left|P_{\mathbf{2}^{j+\ell}, a} \delta_{\boldsymbol{j}+\boldsymbol{\ell}}[f]\right|^{\lambda}(\boldsymbol{x})\right]^{\frac{1}{\lambda}}
$$

Multiplying with the weight $2^{r \cdot j}$ we find the point-wise estimate

$$
2^{\boldsymbol{r} \cdot \boldsymbol{j}}\left|q_{\boldsymbol{j}}^{L}[f](\boldsymbol{x})\right| \lesssim \sum_{\boldsymbol{\ell} \geq 0} 2^{(a \mathbf{1}-\boldsymbol{r}) \cdot \boldsymbol{\ell}} 2^{\boldsymbol{r} \cdot(\boldsymbol{j}+\boldsymbol{\ell})}\left[M\left|P_{\mathbf{2}^{\boldsymbol{j}+\ell, a}} \delta_{\boldsymbol{j}+\boldsymbol{\ell}}[f]\right|^{\lambda}(\boldsymbol{x})\right]^{\frac{1}{\lambda}}
$$

where $\lambda$ is chosen as $L>\frac{1}{\lambda}>\frac{1}{\min \{p, \theta\}}(\lambda=1$ in case $\min \{p, \theta\}>1)$. The parameter $a$ will be fixed later. Now we take the $L_{p}\left(\ell_{\theta}\right)$ (quasi)-norm on both sides. Due to $u$-triangle inequality in $L_{p}\left(\ell_{\theta}\right)$ with $u=\min \{p, \theta, 1\}$ we obtain

$$
\left\|2^{\boldsymbol{r} \cdot \boldsymbol{j}} q_{\boldsymbol{j}}^{L}[f] \mid L_{p}\left(\ell_{\theta}\right)\right\| \lesssim\left(\sum_{\boldsymbol{\ell} \geq 0} 2^{(a \mathbf{1}-\boldsymbol{r}) \cdot \boldsymbol{\ell} u}\left\|2^{\boldsymbol{r} \cdot(\boldsymbol{j}+\boldsymbol{\ell})}\left[M\left|P_{\mathbf{2}^{\boldsymbol{j}+\boldsymbol{\ell}, a}} \delta_{\boldsymbol{j}+\boldsymbol{\ell}}[f]\right|^{\lambda}\right]^{\frac{1}{\lambda}} \mid L_{p}\left(\ell_{\theta}\right)\right\|^{u}\right)^{\frac{1}{u}} .
$$

Since $\lambda<\min \{p, \theta\}$ in case $\min \{p, \theta\} \leq 1$ a trick similar to 4.12 yields

$$
\left\|\left.2^{\boldsymbol{r} \cdot(\boldsymbol{j}+\ell)}\left[M\left|P_{\mathbf{2}^{\boldsymbol{j}+\ell, a}} \delta_{\boldsymbol{j}+\ell}[f]\right|^{\lambda}\right]^{\frac{1}{\lambda}}\left|L_{p}\left(\ell_{\theta}\right)\|=\| 2^{\lambda \boldsymbol{r} \cdot(\boldsymbol{j}+\ell)} M\right| P_{\mathbf{2}^{\boldsymbol{j}+\ell, a}} \delta_{\boldsymbol{j}+\ell}[f]\right|^{\lambda} \mid L_{\frac{p}{\lambda}}\left(\ell_{\frac{\theta}{\lambda}}\right)\right\|^{\frac{1}{\lambda}} .
$$

This allows us to apply Fefferman-Stein maximal inequality (Theorem A.5)

$$
\left\|2^{\boldsymbol{r} \cdot(\boldsymbol{j}+\ell)}\left[M\left|P_{\mathbf{2}^{\boldsymbol{j}+\boldsymbol{\ell}, a}} \delta_{\boldsymbol{j}+\boldsymbol{\ell}}[f]\right|^{\lambda}\right]^{\frac{1}{\lambda}}\left|L_{p}\left(\ell_{\theta}\right)\|\lesssim\| 2^{\boldsymbol{r} \cdot(\boldsymbol{j}+\boldsymbol{\ell})} P_{\mathbf{2}^{\boldsymbol{j}+\boldsymbol{\ell}, a}} \delta_{\boldsymbol{j}+\boldsymbol{\ell}}[f]\right| L_{p}\left(\ell_{\theta}\right)\right\| .
$$

Next we choose $a$ such that $\boldsymbol{r}>a>\max \left\{\frac{1}{p}, \frac{1}{\theta}\right\}$ holds. Then applying Peetre maximal inequality (Theorem 4.5) gives

$$
\left\|2^{r \cdot(\boldsymbol{j}+\ell)} P_{\mathbf{2}^{\ell+j}, a} \delta_{\boldsymbol{\ell}+\boldsymbol{j}}[f]\left|L_{p}\left(\ell_{\theta}\right)\|\lesssim\| 2^{\boldsymbol{r} \cdot(\boldsymbol{j}+\boldsymbol{\ell})} \delta_{\boldsymbol{\ell}+\boldsymbol{j}}[f]\right| L_{p}\left(\ell_{\theta}\right)\right\| .
$$


Obviously, we have

$$
\left\|2^{\boldsymbol{r} \cdot(\boldsymbol{j}+\ell)} \delta_{\ell+\boldsymbol{j}}[f]\left|L_{p}\left(\ell_{\theta}\right)\|\leq\| 2^{\boldsymbol{r} \cdot \boldsymbol{j}} \delta_{\boldsymbol{j}}[f]\right| L_{p}\left(\ell_{\theta}\right)\right\| .
$$

Inserting this into (5.12) yields

$$
\begin{aligned}
\left\|2^{r \cdot \boldsymbol{j}} q_{\boldsymbol{j}}^{L}[f] \mid L_{p}\left(\ell_{\theta}\right)\right\| & \lesssim\left\|2^{\boldsymbol{r} \cdot \boldsymbol{j}} \delta_{\boldsymbol{j}}[f] \mid L_{p}\left(\ell_{\theta}\right)\right\|\left(\sum_{\boldsymbol{\ell} \geq 0} 2^{(a \mathbf{1}-\boldsymbol{r}) \cdot \boldsymbol{\ell} u}\right)^{\frac{1}{u}} \\
& \lesssim\left\|2^{\boldsymbol{r} \cdot \boldsymbol{j}} \delta_{\boldsymbol{j}}[f] \mid L_{p}\left(\ell_{\theta}\right)\right\|
\end{aligned}
$$

where the choice of $a$ ensures the convergence of the series to an absolute constant. Step 2. We prove (i). The equation (5.9) implies

$$
\left\|2^{r \cdot j} q_{j}^{L}[f] \mid L_{p}\left(\ell_{\theta}\right)\right\|<\infty .
$$

Then Theorem 4.6 yields unconditional convergence of the series $\sum_{\boldsymbol{j} \in \mathbb{N}_{0}^{d}} q_{\boldsymbol{j}}^{L}[f]$. We show in case $0<\theta<\infty$

$$
\left\|f-\sum_{|\boldsymbol{j}|_{1}<n} q_{\boldsymbol{j}}^{L}[f] \mid S_{p, \theta}^{\boldsymbol{r}} F\left(\mathbb{T}^{d}\right)\right\| \longrightarrow 0 \quad(n \rightarrow \infty) .
$$

As a consequence of Definition 3.3 trigonometric polynomials are dense in $S_{p, \theta}^{r} F\left(\mathbb{T}^{d}\right)$ if $\theta<\infty$. For that reason we find for every $\varepsilon>0$ a trigonometric polynomial $t$ such that

$$
\left\|f-t \mid S_{p, \theta}^{r} F\left(\mathbb{T}^{d}\right)\right\|<\varepsilon
$$

The $u$-triangle inequality gives

$$
\left\|f-\sum_{|\boldsymbol{j}|_{1}<n} q_{\boldsymbol{j}}^{L}[f]\left|S_{p, \theta}^{\boldsymbol{r}} F\left(\mathbb{T}^{d}\right)\left\|^{u} \leq\right\| f-t\right| S_{p, \theta}^{r} F\left(\mathbb{T}^{d}\right)\right\|^{u}+\left\|t-\sum_{|\boldsymbol{j}|_{1}<n} q_{\boldsymbol{j}}^{L}[f] \mid S_{p, \theta}^{\boldsymbol{r}} F\left(\mathbb{T}^{d}\right)\right\|^{u} .
$$

For $n$ sufficiently large we obtain by Lemma 2.7

$$
t-\sum_{|\boldsymbol{j}|_{1}<n} q_{\boldsymbol{j}}^{L}[f]=\sum_{|\boldsymbol{j}|_{1}<n} q_{\boldsymbol{j}}^{L}(t-f) .
$$

Applying Theorem 4.6 we have

$$
\left\|\sum_{|\boldsymbol{j}|_{1}<n} q_{\boldsymbol{j}}^{L}(t-f) \mid S_{p, \theta}^{\boldsymbol{r}} F\left(\mathbb{T}^{d}\right)\right\| \lesssim\left\|\left(\sum_{|\boldsymbol{j}|_{1}<n} 2^{\theta \boldsymbol{r} \cdot \boldsymbol{j}}\left|q_{\boldsymbol{j}}^{L}(t-f)\right|^{\theta}\right)^{\frac{1}{\theta}}\right\|_{p}
$$

Finally, Step 1 yields

$$
\left\|\left(\sum_{|\boldsymbol{j}|_{1}<n} 2^{\theta \boldsymbol{r} \cdot \boldsymbol{j}}\left|q_{\boldsymbol{j}}^{L}(t-f)\right|^{\theta}\right)^{\frac{1}{\theta}}\right\|_{p} \lesssim\left\|t-f \mid S_{p, \theta}^{\boldsymbol{r}} F\left(\mathbb{T}^{d}\right)\right\|
$$

and hence, there is a constant $C>0$ independent of $n, f$ and $t$ such that

$$
\left\|f-\sum_{|\boldsymbol{j}|_{1}<n} q_{\boldsymbol{j}}^{L}[f] \mid S_{p, \theta}^{\boldsymbol{r}} F\left(\mathbb{T}^{d}\right)\right\| \leq C 2 \varepsilon .
$$

The case $\theta=\infty$ is based on the embedding

$$
S_{p, \infty}^{r} F\left(\mathbb{T}^{d}\right) \hookrightarrow S_{p, p}^{s} F\left(\mathbb{T}^{d}\right) \hookrightarrow S_{p, \nu}^{\tilde{r}} F\left(\mathbb{T}^{d}\right)
$$

with $\boldsymbol{r}>\boldsymbol{s}>\frac{1}{p}, \boldsymbol{s}>\tilde{\boldsymbol{r}}$ and $0<\nu<\infty$ where the density argument from above is applied to $S_{p, p}^{s} F\left(\mathbb{T}^{d}\right)$. 
Remark 5.8. The recent result in [33, Rem. 7.3], see also [34], indicates that a corresponding characterization in case of small smoothness, i.e. $\frac{1}{p}<r \leq \frac{1}{\theta}$ may fail.

Theorem 5.9. Let $0<p, \theta \leq \infty, L>\max \left\{\frac{1}{p}, 1\right\}, \boldsymbol{r}>\frac{1}{p}$.

(i) Then every $f \in S_{p, \theta}^{r} B\left(\mathbb{T}^{d}\right)$ can be represented by

$$
f=\sum_{\boldsymbol{j} \in \mathbb{N}_{0}^{d}} q_{\boldsymbol{j}}^{L}(f),
$$

with unconditional convergence in $S_{p, \theta}^{r} B\left(\mathbb{T}^{d}\right)$ in case $\max \{p, \theta\}<\infty$, and with unconditional convergence in $S_{p, \nu}^{\tilde{\boldsymbol{r}}} B\left(\mathbb{T}^{d}\right)$ for every $\boldsymbol{r}>\tilde{\boldsymbol{r}}$ and $0<\nu \leq \infty$ in case $\max \{p, \theta\}=\infty$.

(ii) There is a constant $C>0$ independent of $f$ such that

$$
\left\|2^{r \cdot j} q_{j}^{L}[f]\left|\ell_{\theta}\left(L_{p}\left(\mathbb{T}^{d}\right)\right)\|\leq C\| f\right| S_{p, \theta}^{r} B\left(\mathbb{T}^{d}\right)\right\|
$$

holds for all $f \in S_{p, \theta}^{r} B\left(\mathbb{T}^{d}\right)$.

Proof. Concerning representation and unconditional convergence we follow the proof of Theorem 5.7 line by line with the obvious modifications for the $B$-case. The inequality in (ii) can be proved by the following arguments. We take the $\ell_{\theta}\left(L_{p}\left(\mathbb{T}^{d}\right)\right.$ ) (quasi)-norm on both sides of the estimate in (5.11). Due to $u$-triangle inequality in $\ell_{\theta}\left(L_{p}\left(\mathbb{T}^{d}\right)\right)$ with $u=\min \{p, \theta, 1\}$ we obtain

$$
\left\|2^{r \cdot \boldsymbol{j}} q_{\boldsymbol{j}}^{L}[f] \mid \ell_{\theta}\left(L_{p}\left(\mathbb{T}^{d}\right)\right)\right\| \lesssim\left(\sum_{\boldsymbol{\ell} \geq 0} 2^{(a \mathbf{1}-\boldsymbol{r}) \cdot \ell u}\left\|2^{\boldsymbol{r} \cdot(\boldsymbol{j}+\ell)}\left[M\left|P_{\mathbf{2}^{j+\ell, a}} \delta_{\boldsymbol{j}+\ell}[f]\right|^{\lambda}\right]^{\frac{1}{\lambda}} \mid \ell_{\theta}\left(L_{p}\left(\mathbb{T}^{d}\right)\right)\right\|^{u}\right)^{\frac{1}{u}}
$$

with $\boldsymbol{r}>a>\frac{1}{p}$ and $0<\lambda<p(\lambda=1$ if $p>1)$. In case $p \leq 1$ a trick similar to 4.12 yields

$$
\| 2^{r \cdot(\boldsymbol{j}+\ell)}\left[M\left|P_{\mathbf{2}^{j+\ell}, a} \delta_{\boldsymbol{j}+\boldsymbol{\ell}}[f]\right|^{\lambda}\right]^{\frac{1}{\lambda}} \mid \ell_{\theta}\left(L_{p}\left(\mathbb{T}^{d}\right) \|=\left(\sum_{\boldsymbol{j} \in \mathbb{N}_{0}^{d}} 2^{\boldsymbol{r} \cdot(\boldsymbol{j}+\ell) \theta}\left\|M\left|P_{\mathbf{2}^{j+\ell}, a} \delta_{\boldsymbol{j}+\boldsymbol{\ell}}[f]\right|^{\lambda}\right\|_{\frac{p}{\lambda}}^{\frac{\theta}{\lambda}}\right)^{\frac{1}{\theta}} .\right.
$$

This allows us to apply Hardy-Littlewood maximal inequality (Theorem A.4). We obtain

$$
\| 2^{r \cdot(\boldsymbol{j}+\ell)}\left[M\left|P_{\mathbf{2}^{\boldsymbol{j}+\boldsymbol{\ell}, a}} \delta_{\boldsymbol{j}+\boldsymbol{\ell}}[f]\right|^{\lambda}\right]^{\frac{1}{\lambda}} \mid \ell_{\theta}\left(L_{p}\left(\mathbb{T}^{d}\right) \| \lesssim\left(\sum_{\boldsymbol{j} \in \mathbb{N}_{0}^{d}} 2^{r \cdot(\boldsymbol{j}+\boldsymbol{\ell}) \theta}\left\|P_{\mathbf{2}^{\boldsymbol{j}+\boldsymbol{\ell}, a}} \delta_{\boldsymbol{j}+\boldsymbol{\ell}}[f]\right\| \|_{p}^{\theta}\right)^{\frac{1}{\theta}} .\right.
$$

Inserting this into (5.13) and applying (non-vector valued) Peetre maximal inequality (Theorem 4.4) gives

$$
\begin{aligned}
\left\|2^{r \cdot \boldsymbol{j}} q_{\boldsymbol{j}}^{L}[f] \mid \ell_{\theta}\left(L_{p}\left(\mathbb{T}^{d}\right)\right)\right\| & \lesssim\left(\sum_{\boldsymbol{\ell} \geq 0} 2^{(a \mathbf{1}-\boldsymbol{r}) \cdot \boldsymbol{\ell} u}\left\|2^{\boldsymbol{r} \cdot(\boldsymbol{j}+\boldsymbol{\ell})} \delta_{\boldsymbol{\ell}+\boldsymbol{j}}[f] \mid \ell_{\theta}\left(L_{p}\left(\mathbb{T}^{d}\right)\right)\right\|^{u}\right)^{\frac{1}{u}} \\
& \leq\left(\sum_{\boldsymbol{\ell} \geq 0} 2^{(a \mathbf{1}-\boldsymbol{r}) \cdot \boldsymbol{\ell} u}\right)^{\frac{1}{u}}\left\|2^{r \cdot \boldsymbol{j}} \delta_{\boldsymbol{j}}[f] \mid \ell_{\theta}\left(L_{p}\left(\mathbb{T}^{d}\right)\right)\right\|,
\end{aligned}
$$

where the term inside the $\ell_{\theta}\left(L_{p}\left(\mathbb{T}^{d}\right)\right)$ norm does not depend any longer on $\ell$. Therefore the sum over $\ell$ converges to a constant depending only on $a, \boldsymbol{r}$ and the dimension $d$. Finally, we obtain

$$
\left\|2^{\boldsymbol{r} \cdot \boldsymbol{j}} q_{\boldsymbol{j}}^{L}[f]\left|\ell_{\theta}\left(L_{p}\left(\mathbb{T}^{d}\right)\right)\|\lesssim\| f\right| S_{p, \theta}^{\boldsymbol{r}} B\left(\mathbb{T}^{d}\right)\right\| .
$$




\subsection{The case of the Dirichlet kernel where $L=1$}

In this subsection we study sampling representations based on the Dirichlet kernel $K_{\pi, j}^{1}$. Its slow decay causes some difficulties. We define an auxiliary kernel

$$
\tilde{K}^{2}(x):=\sqrt{2 \pi} \mathcal{F}^{-1}\left(4 \chi_{\left[-\frac{5}{8}, \frac{5}{8}\right]} * \chi_{\left[-\frac{1}{8}, \frac{1}{8}\right]}\right)(x)=16 \frac{\sin \left(\frac{5}{8} x\right) \sin \left(\frac{1}{8} x\right)}{x^{2}} \in L_{1}\left(\mathbb{R}^{d}\right)
$$

and its periodization

$$
\tilde{K}_{\pi, j}^{2}(x):=\sum_{k=-\infty}^{\infty} \tilde{K}^{2}\left(2^{j}(x+2 \pi k)\right) .
$$

Similar to Lemma 2.6 we can show for $|x|<\pi$ the following decay property

$$
\left|\tilde{K}_{\pi, j}^{2}(x)\right| \lesssim \frac{1}{\left(1+2^{j}|x|\right)^{2}}
$$

Note, that the corresponding operator $\tilde{I}_{j}^{2}$ defined via (1.11) is a sampling but not an interpolation operator. However, Lemma 2.2 still holds true. According to Subsection 2.2 we define the multivariate sampling operator $\tilde{I}_{\boldsymbol{j}}^{2} f$ based on the tensorized kernel $\tilde{K}_{\pi^{d}, \boldsymbol{j}}^{2}$.

The following formula is a counterpart of a similar formula used by Temlyakov in [42, Lem. I.6.2]. Taking (1.12) into account we denote

$$
\mathcal{D}_{j}^{1}=\mathcal{D}_{j_{1}}^{1} \otimes \cdots \otimes \mathcal{D}_{j_{d}}^{1} \quad, \quad j \in \mathbb{N}_{0}^{d} .
$$

Lemma 5.10. Let $f \in C\left(\mathbb{T}^{d}\right)$. Then

$$
I_{\boldsymbol{j}}^{1} f=(2 \pi)^{-d} \mathcal{D}_{\boldsymbol{j}}^{1} * \tilde{I}_{\boldsymbol{j}}^{2} f
$$

for all $\boldsymbol{j} \in \mathbb{N}_{0}^{d}$.

Proof. We prove the identity by comparing the Fourier series for arbitrary continuous functions $f$. (2.5) implies

$$
\widehat{I_{j}^{1} f}(\boldsymbol{\ell})=\left(\prod_{i \in[d]} \chi_{\left[-2^{j_{i}-1}, 2^{j_{i}}-1-1\right]}\left(\ell_{i}\right)\right) \sum_{\boldsymbol{u} \in A_{j}} f\left(\boldsymbol{x}_{\boldsymbol{u}}^{j}\right) e^{-i \boldsymbol{x}_{\boldsymbol{u}}^{j} \cdot \boldsymbol{\ell}} .
$$

Additionally, the same computation as used in Lemma 2.2 shows

$$
\widehat{\tilde{I}_{\boldsymbol{j}}^{2}[f]}(\boldsymbol{\ell})=\frac{1}{(2 \pi)^{d / 2}}\left(\prod_{i=1}^{d} \mathcal{F} \tilde{K}^{2}\left(\frac{\ell_{i}}{2^{j_{i}}}\right)\right) \sum_{\boldsymbol{u} \in A_{j}} f\left(\boldsymbol{x}_{\boldsymbol{u}}^{j}\right) e^{-i \boldsymbol{x}_{\boldsymbol{u}}^{j} \cdot \boldsymbol{\ell}} .
$$

Clearly,

$$
(2 \pi)^{-d} \widehat{\mathcal{D}_{j}^{1} * \tilde{I}_{j}^{2}} f(\ell)=\widehat{\mathcal{D}_{j}^{1}}(\ell) \widehat{\tilde{I}_{j}^{2} f}(\ell)=\widehat{\mathcal{D}_{j}^{1}}(\ell) \sum_{\boldsymbol{u} \in A_{j}} f\left(\boldsymbol{x}_{\boldsymbol{u}}^{j}\right) e^{-i \boldsymbol{x}_{\boldsymbol{u}}^{j} \cdot \boldsymbol{\ell}}
$$

since

$$
\mathcal{F} \tilde{K}^{2}\left(\frac{\ell_{i}}{2^{j_{i}}}\right)=\sqrt{2 \pi}
$$

for $\ell_{i} \in\left[-2^{j_{i}}, 2^{j_{i}}\right), i \in[d]$. Comparing (5.16) and (5.17) yields the claim. 
Lemma 5.11. Let $\boldsymbol{\ell}, \boldsymbol{j} \in \mathbb{N}_{0}^{d}, a>0$ and $1 / 2<\lambda \leq 1$. Furthermore, let $f \in C\left(\mathbb{T}^{d}\right)$. Then

$$
\left|\tilde{I}_{\boldsymbol{j}}^{2}[f](\boldsymbol{x})\right| \lesssim 2^{a|\ell|_{1}}\left[M\left|P_{\mathbf{2}^{j+\ell}, a} f\right|^{\lambda}(\boldsymbol{x})\right]^{\frac{1}{\lambda}}
$$

holds with a constant independent of $\boldsymbol{\ell}, \boldsymbol{j}, \boldsymbol{x}$ and $f$.

Proof. We refer to the proof of Proposition 5.6. Recognizing, that the only property of $\tilde{I}_{j}^{2}$ we need is the decay of the underlying kernel $\tilde{K}_{\pi^{d}, j}^{2}$ provided in (5.14).

Remark 5.12. (i) The estimates in Lemmas [5.6, 5.11 are pointwise and very useful for $L_{p}\left(\mathbb{T}^{d}, \ell_{\theta}\right)$ estimates. In case one is interested in (scalar) $L_{p}$ estimates, similar as in 42. Lem. I.6.2], then Lemmas 5.6 and 5.11 together with the maximal inequalities Theorems A.4. 4.4 imply for $0<p \leq \infty, L>\max \{1 / p, 1\}$ and any $a>1 / p$

$$
\left\|I_{j}^{L} f\right\|_{p} \lesssim L, a 2^{|\ell|_{1} a}\|f\|_{p} \quad, \quad f \in \mathcal{T}_{j+\ell}^{0}
$$

(similar for $\left.\tilde{I}_{\boldsymbol{j}}^{2}\right)$

(ii) There is a different technique based on periodic versions of Plancherel-Polya inequalities (Marcinkiewicz-Zygmund inequalities) for $0<p \leq \infty$, see [31, Thms. 6,10]. A straight-forward modification of the argument in [31, Lem. 13,(ii)] gives for $0<p \leq \infty$ and $L>\max \{1 / p, 1\}$

$$
\left\|I_{j}^{L} f\right\|_{p} \lesssim p 2^{|\ell|_{1} / p}\|f\|_{p} \quad, \quad f \in \mathcal{T}_{j+\ell}^{0}
$$

(similar for $\tilde{I}_{j}^{2}$ ). In case $L=2$ (de la Vallée Poussin) this yields an extension of 4, Lem. I.6.2] to the range $1 / 2<p \leq \infty$.

(iii) By Lemma 5.10 and the uniform boundedness of the multivariate Fourier partial sum operator in $L_{p}\left(\mathbb{T}^{d}\right), 1<p<\infty$, we obtain from (5.18) and (5.19) corresponding estimates also for $\left\|I_{j}^{1} f\right\|_{p}$.

Theorem 5.13. Let $1<p, \theta<\infty$ and $\boldsymbol{r}>\max \left\{\frac{1}{p}, \frac{1}{\theta}\right\}$.

(i) Then every $f \in S_{p, \theta}^{r} F\left(\mathbb{T}^{d}\right)$ admits the representation

$$
f=\sum_{\boldsymbol{j} \in \mathbb{N}_{0}^{d}} q_{\boldsymbol{j}}^{1}[f],
$$

with unconditional convergence in $S_{p, \theta}^{r} F\left(\mathbb{T}^{d}\right)$.

(ii) There is a constant $C>0$ independent of $f$ such that

$$
\left\|2^{r \cdot j} q_{j}^{1}(f)\left|L_{p}\left(\ell_{\theta}\right)\|\leq C\| f\right| S_{p, \theta}^{r} F\left(\mathbb{T}^{d}\right)\right\|
$$

holds for all $f \in S_{p, \theta}^{r} F\left(\mathbb{T}^{d}\right)$.

Proof. The proof of (i) is similar to Theorem 5.7, (i). We prove (ii) here. Inserting the decomposition (3.2), applying triangle inequality and afterwards Proposition 2.10 gives

$$
\left\|2^{\boldsymbol{r} \cdot \boldsymbol{j}} q_{\boldsymbol{j}}^{1}[f]\left|L_{p}\left(\ell_{\theta}\left(\mathbb{N}_{0}^{d}\right)\right)\left\|\lesssim \sum_{\boldsymbol{\ell} \geq 0} 2^{-\boldsymbol{\ell} \cdot \boldsymbol{r}}\right\| 2^{\boldsymbol{r} \cdot(\boldsymbol{j}+\boldsymbol{\ell})} q_{\boldsymbol{j}}^{1}\left[\delta_{\boldsymbol{j}+\boldsymbol{\ell}}[f]\right]\right| L_{p}\left(\ell_{\theta}\left(\mathbb{N}_{0}^{d}\right)\right)\right\| .
$$


The relation in (5.7) shows

$$
\left\|2^{\boldsymbol{r} \cdot \boldsymbol{j}} q_{\boldsymbol{j}}^{1}[f]\left|L_{p}\left(\ell_{\theta}\left(\mathbb{N}_{0}^{d}\right)\right)\left\|\lesssim \sum_{\boldsymbol{b} \in\{-1,0\}^{d}} \sum_{\boldsymbol{\ell} \geq 0} 2^{-\boldsymbol{\ell} \cdot \boldsymbol{r}}\right\| 2^{\boldsymbol{r} \cdot(\boldsymbol{j}+\boldsymbol{\ell})} I_{\boldsymbol{j}+\boldsymbol{b}}^{1}\left[\delta_{\boldsymbol{j}+\ell}[f]\right]\right| L_{p}\left(\ell_{\theta}\left(\mathbb{N}_{0}^{d}\right)\right)\right\|
$$

Hence, Lemma 5.10 yields

$$
\left\|2^{r \cdot \boldsymbol{j}} q_{\boldsymbol{j}}^{1}[f]\left|L_{p}\left(\ell_{\theta}\left(\mathbb{N}_{0}^{d}\right)\right)\left\|\lesssim \sum_{\boldsymbol{b} \in\{-1,0\}^{d}} \sum_{\boldsymbol{\ell} \geq 0} 2^{-\ell \cdot \boldsymbol{r}}\right\| 2^{\boldsymbol{r} \cdot(\boldsymbol{j}+\ell)} \mathcal{D}_{\boldsymbol{j}+\boldsymbol{b}}^{1} * \tilde{I}_{\boldsymbol{j}+\boldsymbol{b}}^{2}\left[\delta_{\boldsymbol{j}+\boldsymbol{\ell}}[f]\right]\right| L_{p}\left(\ell_{\theta}\left(\mathbb{N}_{0}^{d}\right)\right)\right\|
$$

Lizorkin presented in [22, p. 241, Thm. 5] a theorem on Fourier multipliers for the $L_{p}\left(\ell_{\theta}\right)$ situation. The result in [32, Thm. 3.4.2] transfers this to the periodic setting. Referring to a comment in [43, 2.5.4] the Fourier partial sum with respect to a parallelepiped fulfills the requirements of this theorem and we get rid of $\mathcal{D}_{\boldsymbol{j}+\boldsymbol{b}}^{1}$ in (5.20). This gives

$$
\left\|2^{\boldsymbol{r} \cdot \boldsymbol{j}} q_{\boldsymbol{j}}^{1}[f]\left|L_{p}\left(\ell_{\theta}\left(\mathbb{N}_{0}^{d}\right)\right)\left\|\lesssim \sum_{\boldsymbol{b} \in\{-1,0\}^{d}} \sum_{\boldsymbol{\ell} \geq 0} 2^{-\boldsymbol{\ell} \cdot \boldsymbol{r}}\right\| 2^{\boldsymbol{r} \cdot(\boldsymbol{j}+\boldsymbol{\ell})} \tilde{I}_{\boldsymbol{j}+\boldsymbol{b}}^{2} \delta_{\boldsymbol{j}+\boldsymbol{\ell}}[f]\right| L_{p}\left(\ell_{\theta}\left(\mathbb{N}_{0}^{d}\right)\right)\right\|
$$

Lemma 5.11 with $\lambda=1$ yields

$$
\left\|2^{\boldsymbol{r} \cdot \boldsymbol{j}} q_{\boldsymbol{j}}^{1}[f]\left|L_{p}\left(\ell_{\theta}\left(\mathbb{N}_{0}^{d}\right)\right)\left\|\lesssim \sum_{\boldsymbol{\ell} \geq 0} 2^{\ell \cdot(a \mathbf{1}-\boldsymbol{r})}\right\| 2^{\boldsymbol{r} \cdot(\boldsymbol{j}+\ell)} M\right| P_{\mathbf{2}^{j+\ell}, a} f_{j+\ell}|(\boldsymbol{x})| L_{p}\left(\ell_{\theta}\left(\mathbb{N}_{0}^{d}\right)\right)\right\| .
$$

We finish the proof by following the estimates in the proof of Theorem 5.7 beginning from (5.12).

Theorem 5.14. Let $1<p<\infty, 0<\theta \leq \infty$ and $\boldsymbol{r}>\frac{1}{p}$.

(i) Then every $f \in S_{p, \theta}^{r} B\left(\mathbb{T}^{d}\right)$ can be represented by

$$
f=\sum_{\boldsymbol{j} \in \mathbb{N}_{0}^{d}} q_{\boldsymbol{j}}^{1}(f),
$$

with unconditional convergence in $S_{p, \theta}^{r} B\left(\mathbb{T}^{d}\right)$ in case $\theta<\infty$, and with unconditional convergence in $S_{p, \nu}^{\tilde{\boldsymbol{r}}} B\left(\mathbb{T}^{d}\right)$ for every $\boldsymbol{r}>\tilde{\boldsymbol{r}}$ and $0<\nu \leq \infty$ in case $\theta=\infty$.

(ii) There is a constant $C>0$ independent of $f$ such that

$$
\left\|2^{r \cdot j} q_{j}^{1}[f]\left|\ell_{\theta}\left(L_{p}\left(\mathbb{T}^{d}\right)\right)\|\leq C\| f\right| S_{p, \theta}^{r} B\left(\mathbb{T}^{d}\right)\right\|
$$

holds for all $f \in S_{p, \theta}^{\boldsymbol{r}} B\left(\mathbb{T}^{d}\right)$.

Proof. To prove (i) we follow the proof of Theorem [5.9, (i). The assertion (ii) can be obtained following the proof of Theorem 5.13 where we replace $\left\|\cdot \mid L_{p}\left(\ell_{\theta}\left(\mathbb{N}_{0}\right)\right)\right\|$ by $\left\|\cdot \mid \ell_{\theta}\left(L_{p}\left(\mathbb{T}^{d}\right)\right)\right\|$. Now we use the estimates in Remark (5.18), (5.19) from Remark 5.12.

Remark 5.15. Similar (but not nested) Dirichlet kernels were studied in [2] connected with sampling representations in case $p=\theta=2$. 


\section{Interpolation on Smolyak grids}

In this section we analyze a direction-wise modified version of Smolyak's algorithm, cf. (1.3), given by

$$
T_{m}^{L, \boldsymbol{\eta}} f:=\sum_{\frac{1}{\eta_{1}} \boldsymbol{\eta} \cdot \boldsymbol{j} \leq m} q_{\boldsymbol{j}}^{L}[f] .
$$

The parameter $\boldsymbol{\eta}>0$ allows to control the level of refinement in single directions. A comparatively large value of $\boldsymbol{\eta}$ in the $s$-th component ends up in a small refinement in the $s$-th direction. The interpolation operator $T_{m}^{L, \boldsymbol{\eta}} f$ maps a continuous function to a trigonometric polynomial with frequencies in an anisotropic hyperbolic cross

$$
A H_{m}^{d, \boldsymbol{\eta}}:=\bigcup_{\left\{\boldsymbol{j}: \frac{1}{\eta_{1}} \boldsymbol{\eta} \cdot \boldsymbol{j} \leq m\right\}} \mathcal{P}_{j}^{0} .
$$

According to Lemma 2.8 the operator $T_{m}^{L, \boldsymbol{\eta}}$ interpolates functions on an anisotropic sparse grid

$$
A G_{m}^{d, \boldsymbol{\eta}}:=\bigcup_{\frac{1}{\eta_{1}} \boldsymbol{\eta} \cdot \boldsymbol{j} \leq m}\left\{\boldsymbol{x}_{\boldsymbol{u}}^{\boldsymbol{j}}: \boldsymbol{u} \in \mathbb{Z}^{d},-2^{j_{i}-1} \leq u_{i} \leq 2^{j_{i}-1}-1, i \in[d]\right\} .
$$

Lemma 6.1. Let $\boldsymbol{\eta} \in \mathbb{R}^{d}$ with

$$
0<\eta_{1}=\ldots=\eta_{\mu}<\eta_{\mu+1} \leq \ldots \leq \eta_{d}<\infty
$$

Then

$$
\left|A G_{m}^{d, \boldsymbol{\eta}}\right| \asymp m^{\mu-1} 2^{m}
$$

holds for all $m \geq 1$.

Proof. Due to (6.2) an upper bound for the cardinality of $A G_{m}^{d, \boldsymbol{\eta}}$ is provided by

$$
\left|A G_{m}^{d, \boldsymbol{\eta}}\right| \leq \sum_{\frac{1}{\eta_{1}} \boldsymbol{\eta} \cdot \boldsymbol{j} \leq m} 2^{|\boldsymbol{j}|_{1}}
$$

Therefore Lemma B.2 in the appendix provides the upper bound in (6.3). A trivial lower bound of $2^{m}$ is provided by simply counting the sampling nodes of $q_{j}[f]$ of the level $j=(m, 0, \ldots, 0)$. A sharp bound can be obtained by using reproduction properties of $T_{m}^{L, \boldsymbol{\eta}}$ for trigonometric polynomials (cf. Lemma 2.7]) with frequencies in $A H_{m}^{d, \boldsymbol{\eta}}$. The dimension of $A H_{m}^{d, \boldsymbol{\eta}}$ is given by $\sum_{\frac{1}{\eta_{1}} \boldsymbol{\eta} \cdot \boldsymbol{j} \leq m} 2^{|\boldsymbol{j}|_{1}}$.

Remark 6.2. Comparing this estimate to uniformly refined sparse grids $(\boldsymbol{\eta}=\mathbf{1})$ we recognize that the underlying dimension of the space plays no role for the asymptotic bound. The dimension dependence is replaced by the $\mu$ largest refinement directions.

Theorem 6.3. Let $0<p<q<\infty$ and $0<\theta \leq \infty$. Additionally let $L>\frac{1}{q}$ and the smoothness vector $\boldsymbol{r}>\frac{1}{p}$ with (1.5). Then

$$
\left\|f-T_{m}^{L, \boldsymbol{\eta}} f\right\|_{q} \lesssim 2^{-m\left(r_{1}-\frac{1}{p}+\frac{1}{q}\right)}\left\|f \mid S_{p, \theta}^{\boldsymbol{r}} F\left(\mathbb{T}^{d}\right)\right\|
$$

holds for all $m>0$. The operator generating vector $\boldsymbol{\eta} \in \mathbb{R}^{d}$ is chosen as $\boldsymbol{\eta}=\boldsymbol{r}-\frac{1}{p}+\frac{1}{q}$. 
Proof. We start expanding $f$ into the series (5.8). This allows us to estimate

$$
\left\|f-T_{m}^{L, \boldsymbol{\eta}} f\right\|_{q} \leq\left\|\sum_{\frac{1}{\eta_{1}} \boldsymbol{\eta} \cdot \boldsymbol{j}>m}\left|q_{\boldsymbol{j}}[f]\right|\right\|_{q} \leq 2^{-\left(r_{1}-\frac{1}{p}+\frac{1}{q}\right) m}\left\|\sum_{\boldsymbol{j} \in \mathbb{N}_{0}^{d}} 2^{\left(\boldsymbol{r}-\frac{1}{p}+\frac{1}{q}\right) \cdot \boldsymbol{j}}\left|q_{\boldsymbol{j}}[f]\right|\right\|_{q} .
$$

We choose some parameters. Since $L>\frac{1}{q}+1$ we find $\tilde{q} \in \mathbb{R}$ with $p<\tilde{q}<q$ such that $L>\frac{1}{\tilde{q}}+1$ is fulfilled. Let $\tilde{\mathbf{r}}:=\boldsymbol{r}-\frac{1}{p}+\frac{1}{\tilde{q}}$. Applying Lemma 4.7 yields

$$
\left\|f-T_{m}^{L, \boldsymbol{\eta}} f\right\|_{q} \lesssim 2^{-\left(r_{1}-\frac{1}{p}+\frac{1}{q}\right) m}\left\|\sup _{\boldsymbol{j} \in \mathbb{N}_{0}^{d}} 2^{\tilde{\mathbf{r}} \cdot \boldsymbol{j}}\left|q_{\boldsymbol{j}}[f]\right|\right\|_{\tilde{q}}
$$

Theorem 5.7 yields

$$
\left\|f-T_{m}^{L, \boldsymbol{\eta}} f\right\|_{q} \lesssim 2^{-\left(r_{1}-\frac{1}{p}+\frac{1}{q}\right) m}\left\|f \mid S_{\tilde{\boldsymbol{q}}, \infty}^{\tilde{\mathbf{r}}} F\left(\mathbb{T}^{d}\right)\right\| .
$$

Finally, using the diagonal embedding stated in Lemma 3.5, (vi) gives

$$
\left\|f-T_{m}^{L, \boldsymbol{\eta}} f\right\|_{q} \lesssim 2^{-\left(r_{1}-\frac{1}{p}+\frac{1}{q}\right) m}\left\|f \mid S_{p, \theta}^{r} F\left(\mathbb{T}^{d}\right)\right\|,
$$

which finishes the proof.

For $\theta=2$ we can reproduce and generalize a result due to Temlyakov [41].

Theorem 6.4. Let $0<p<\infty, 0<\theta \leq \infty$. Additionally, let $L \geq 1$ and the smoothness vector $r>\frac{1}{p}$ with (1.5). Then

$$
\left\|f-T_{m}^{L, \boldsymbol{\eta}} f\right\|_{\infty} \lesssim m^{(\mu-1)\left(1-\frac{1}{p}\right)+} 2^{-m\left(r_{1}-\frac{1}{p}\right)}\left\|f \mid S_{p, \theta}^{r} F\left(\mathbb{T}^{d}\right)\right\|
$$

holds for all $m \geq 0$. The operator generating vector $\boldsymbol{\eta} \in \mathbb{R}^{d}$ is chosen as $\boldsymbol{\eta}=\boldsymbol{\nu}-\frac{1}{p}$, where $\boldsymbol{\nu} \in \mathbb{R}^{d}$ with

$$
r_{s}=\nu_{s}, \quad s=1, \ldots, \mu \quad \text { and } \quad r_{1}<\nu_{s}<r_{s}, \quad s=\mu+1, \ldots, d .
$$

Proof. Step 1. We prove

$$
\left\|f-T_{m}^{L, \boldsymbol{\eta}} f\right\|_{\infty} \lesssim\left\|f \mid S_{\tilde{p}, p}^{\boldsymbol{r}-\frac{1}{p}+\frac{1}{\tilde{p}}} B\left(\mathbb{T}^{d}\right)\right\| \begin{cases}2^{-m\left(r_{1}-\frac{1}{p}\right)} & : \quad 0<p \leq 1, \\ m^{(\mu-1)\left(1-\frac{1}{p}\right)} 2^{-m\left(r_{1}-\frac{1}{p}\right)} & : \quad p>1,\end{cases}
$$

where $\tilde{p}$ is chosen such that $\max \{p, 1\}<\tilde{p}<\infty$ is fulfilled. Expanding into (5.8) and using triangle inequality yields

$$
\left\|f-T_{m}^{L, \boldsymbol{\eta}} f\right\|_{\infty}=\left\|\sum_{\frac{1}{\eta_{1}} \boldsymbol{\eta} \cdot \boldsymbol{j}>m} q_{\boldsymbol{j}}^{L}[f]\right\|_{\infty} \leq \sum_{\frac{1}{\eta_{1}} \boldsymbol{\eta} \cdot \boldsymbol{j}>m}\left\|q_{\boldsymbol{j}}^{L}[f]\right\|_{\infty}
$$

We have to distinguish the cases $0<p \leq 1$ and the case $p>1$. We start with $0<p \leq 1$. The elementary embedding $\ell_{p}\left(\mathbb{N}_{0}^{d}\right) \hookrightarrow \ell_{1}\left(\mathbb{N}_{0}^{d}\right)$ yields

$\left\|f-T_{m}^{L, \boldsymbol{\eta}} f\right\|_{\infty} \leq 2^{-m\left(r_{1}-\frac{1}{p}\right)} \sum_{\frac{1}{\eta_{1}} \boldsymbol{\eta} \cdot \boldsymbol{j}>m} 2^{\left(\boldsymbol{r}-\frac{1}{p}\right) \cdot \boldsymbol{j}}\left\|q_{\boldsymbol{j}}^{L}[f]\right\|_{\infty} \leq 2^{-m\left(r_{1}-\frac{1}{p}\right)}\left(\sum_{\frac{1}{\eta_{1}} \boldsymbol{\eta} \cdot \boldsymbol{j}>m} 2^{p\left(\boldsymbol{r}-\frac{1}{p}\right) \cdot \boldsymbol{j}}\left\|q_{\boldsymbol{j}}^{L}[f]\right\|_{\infty}^{p}\right)^{\frac{1}{p}}$. 
In case $p>1$ we apply Hölder's inequality with $1=\frac{1}{p}+\frac{1}{p^{\prime}}$ and obtain

$$
\left\|f-T_{m}^{L, \boldsymbol{\eta}} f\right\|_{\infty} \leq\left(\sum_{\frac{1}{\eta_{1}} \boldsymbol{\eta} \cdot \boldsymbol{j}>m} 2^{-p^{\prime}\left(\boldsymbol{r}-\frac{1}{p}\right) \cdot \boldsymbol{j}}\right)^{\frac{1}{p^{\prime}}}\left(\sum_{\frac{1}{\eta_{1}} \boldsymbol{\eta} \cdot \boldsymbol{j}>m} 2^{p\left(\boldsymbol{r}-\frac{1}{p}\right) \cdot \boldsymbol{j}}\left\|q_{\boldsymbol{j}}^{L}[f]\right\|_{\infty}^{p}\right)^{\frac{1}{p}} .
$$

Lemma B.1 yields

$$
\left\|f-T_{m}^{L, \boldsymbol{\eta}} f\right\|_{\infty} \leq m^{(\mu-1)(1-p)} 2^{-m\left(r_{1}-\frac{1}{p}\right)}\left(\sum_{\frac{1}{\eta_{1}} \boldsymbol{\eta} \cdot \boldsymbol{j}>m} 2^{p\left(\boldsymbol{r}-\frac{1}{p}\right) \cdot \boldsymbol{j}}\left\|q_{\boldsymbol{j}}^{L}[f]\right\|_{\infty}^{p}\right)^{\frac{1}{p}} .
$$

Nikolskij's inequality (special case of Lemma 4.7) gives

$$
\left\|f-T_{m}^{L, \boldsymbol{\eta}} f\right\|_{\infty} \leq m^{(\mu-1)(1-p)} 2^{-m\left(r_{1}-\frac{1}{p}\right)}\left(\sum_{\frac{1}{\eta_{1}} \boldsymbol{\eta} \cdot \boldsymbol{j}>m} 2^{p\left(\boldsymbol{r}-\frac{1}{p}+\frac{1}{\tilde{p}}\right) \cdot \boldsymbol{j}}\left\|q_{\boldsymbol{j}}^{L}[f]\right\|_{\tilde{p}}^{p}\right)^{\frac{1}{p}} .
$$

In both cases Theorem 5.9 yields (6.4).

Step 2. The Jawerth-Franke type embedding implies

$$
S_{p, \theta}^{r} F\left(\mathbb{T}^{d}\right) \hookrightarrow S_{\tilde{p}, p}^{\boldsymbol{r}-\frac{1}{p}+\frac{1}{\tilde{p}}} B\left(\mathbb{T}^{d}\right)
$$

(cf. Lemma 3.6). Applying this we obtain

$$
\left\|f-T_{m}^{L, \boldsymbol{\eta}} f\right\|_{\infty} \lesssim m^{(\mu-1)\left(1-\frac{1}{p}\right)_{+}} 2^{-m\left(r_{1}-\frac{1}{p}\right)}\left\|f \mid S_{p, \theta}^{r} F\left(\mathbb{T}^{d}\right)\right\|,
$$

which proves the claim.

Remark 6.5. It is remarkable that Theorem 6.3 allows to use the Smolyak algorithm based on the classical (nested) trigonometric interpolation (Dirichlet kernel) in case $1<q \leq \infty$ although $p<q$ may be less than one. A similar observation has been made recently in [2, Rem. 6.12].

In the remainder of this section we deal with Besov spaces $S_{p, \theta}^{r} B\left(\mathbb{T}^{d}\right)$. A similar result as stated here was obtained by Dinh Dũng in [10], see also [7]. We contribute the case $\min \{p, \theta\}<$ 1 for the Fourier analytical approach and allow the Dirichlet kernel $(L=1)$ for $q>1$.

Theorem 6.6. Let $0<p<q<\infty$ and $0<\theta \leq \infty$. Additionally let $L>\frac{1}{q}$ and the smoothness vector $\boldsymbol{r}>\frac{1}{p}$ with (1.5). Then

$$
\left\|f-T_{m}^{L, \boldsymbol{\eta}} f\right\|_{q} \lesssim 2^{-m\left(r_{1}-\frac{1}{p}+\frac{1}{q}\right)} m^{(\mu-1)\left(\frac{1}{q}-\frac{1}{\theta}\right)+}\left\|f \mid S_{p, \theta}^{\boldsymbol{r}} B\left(\mathbb{T}^{d}\right)\right\|
$$

holds for all $m>0$. The operator generating vector $\boldsymbol{\eta} \in \mathbb{R}^{d}$ is chosen as $\boldsymbol{\eta}=\boldsymbol{\nu}-\frac{1}{p}+\frac{1}{q}$, where $\boldsymbol{\nu} \in \mathbb{R}^{d}$ with

$$
r_{s}=\nu_{s}, \quad s=1, \ldots, \mu \quad \text { and } \quad r_{1}<\nu_{s}<r_{s}, \quad s=\mu+1, \ldots, d
$$


Proof. First we prove the case $q>1$ with $\theta<\infty$. We find $\tilde{q}<q$ such that $L>\frac{1}{\tilde{q}}>\frac{1}{q}$ holds. The Jawerth-Franke embedding $S_{\tilde{\tilde{q}}, q}^{\frac{1}{\tilde{q}}-\frac{1}{q}} B\left(\mathbb{T}^{d}\right) \subset L_{q}\left(\mathbb{T}^{d}\right)$ (cf. Lemma 3.6) yields

$$
\left\|f-T^{L, \boldsymbol{\eta}} f\right\|_{q} \leq\left\|f-T^{L, \boldsymbol{\eta}} f \mid S_{\tilde{q}, q}^{\frac{1}{\tilde{\tilde{q}}}-\frac{1}{q}} B\left(\mathbb{T}^{d}\right)\right\|
$$

Expanding $f$ into the series (5.8) and applying Theorem 4.8 gives

$$
\left\|f-T^{L, \boldsymbol{\eta}} f\right\|_{q} \leq\left(\sum_{\frac{1}{\eta_{1}} \eta \cdot \boldsymbol{j}>m} 2^{q \boldsymbol{j} \cdot \mathbf{1}\left(\frac{1}{\tilde{q}}-\frac{1}{q}\right)}\left\|q_{\boldsymbol{j}}[f]\right\|_{\tilde{q}}^{q}\right)^{\frac{1}{q}} .
$$

In case $\infty>\theta>q$ this can be estimated by using Hölder's inequality

$$
\left\|f-T^{L, \boldsymbol{\eta}} f\right\|_{q} \leq\left(\sum_{\frac{1}{\eta_{1}} \boldsymbol{\eta} \cdot \boldsymbol{j}>m} 2^{-\frac{q \theta}{\theta-q} \boldsymbol{j} \cdot\left(\boldsymbol{r}-\frac{1}{p}+\frac{1}{q}\right)}\right)^{\frac{1}{q}-\frac{1}{\theta}}\left(\sum_{\frac{1}{\eta_{1}} \boldsymbol{\eta} \cdot \boldsymbol{j}>m} 2^{\theta \boldsymbol{j} \cdot\left(\boldsymbol{r}-\left(\frac{1}{p}-\frac{1}{\tilde{q}}\right)\right)}\left\|q_{\boldsymbol{j}}[f]\right\|_{\tilde{q}}^{\theta}\right)^{\frac{1}{\theta}} .
$$

The estimate for the sum in Lemma B.1 gives

$$
\left\|f-T^{L, \boldsymbol{\eta}} f\right\|_{q} \leq 2^{-m\left(r_{1}-\frac{1}{p}+\frac{1}{q}\right)} m^{(\mu-1)\left(\frac{1}{q}-\frac{1}{\theta}\right)}\left(\sum_{\frac{1}{\eta_{1}} \boldsymbol{\eta} \cdot \boldsymbol{j}>m} 2^{\theta \boldsymbol{j} \cdot\left(\boldsymbol{r}-\left(\frac{1}{p}-\frac{1}{\tilde{q}}\right)\right)}\left\|q_{\boldsymbol{j}}[f]\right\|_{\tilde{q}}^{\theta}\right)^{\frac{1}{\theta}} .
$$

In case $\theta \leq q$ we use the embedding $\ell_{\theta} \hookrightarrow \ell_{q}$ and obtain

$$
\left\|f-T^{L, \boldsymbol{\eta}} f\right\|_{q} \leq 2^{-m\left(r_{1}-\frac{1}{p}+\frac{1}{q}\right)}\left(\sum_{\frac{1}{\eta_{1}} \boldsymbol{\eta} \cdot \boldsymbol{j}>m} 2^{\theta \boldsymbol{j} \cdot\left(\boldsymbol{r}-\left(\frac{1}{p}-\frac{1}{\tilde{q}}\right)\right)}\left\|q_{\boldsymbol{j}}[f]\right\|_{\tilde{q}}^{\theta}\right)^{\frac{1}{\theta}} .
$$

Theorem 5.9 allows to estimate

$$
\left\|f-T^{L, \boldsymbol{\eta}} f\right\|_{q} \leq 2^{-m\left(r_{1}-\frac{1}{p}+\frac{1}{q}\right)} m^{(\mu-1)\left(\frac{1}{q}-\frac{1}{\theta}\right)+}\left\|f \mid S_{\tilde{q}, \theta}^{\boldsymbol{r}-\left(\frac{1}{p}-\frac{1}{\tilde{q})}\right.} B\left(\mathbb{T}^{d}\right)\right\| .
$$

Finally, the diagonal embedding stated in Lemma 3.5, (vi) yields

$$
\left\|f-T^{L, \boldsymbol{\eta}} f\right\|_{q} \leq 2^{-m\left(r_{1}-\frac{1}{p}+\frac{1}{q}\right)} m^{(d-1)\left(\frac{1}{q}-\frac{1}{\theta}\right)+}\left\|f \mid S_{p, \theta}^{\boldsymbol{r}} B\left(\mathbb{T}^{d}\right)\right\| .
$$

The case $q \leq 1$ is simpler. We expand $f$ into the series (5.8). Then $q$-triangle inequality yields

$$
\left\|f-T_{m}^{L, \boldsymbol{\eta}} f\right\|_{q} \leq\left(\sum_{\frac{1}{\eta_{1}} \boldsymbol{\eta} \cdot \boldsymbol{j}>m}\left\|q_{j}[f]\right\|_{q}^{q}\right)^{\frac{1}{q}} .
$$

The same case study as in the lines after (6.5) with $\tilde{q}=q$ finishes the proof. As usual in case $\theta=\infty$ we have to replace the corresponding sum by sup.

\section{Linear sampling recovery}

In this section we consider the optimality of convergence rates for linear sampling algorithms in case of Triebel-Lizorkin and Hölder-Nikolskij spaces with mixed smoothness, we abbreviate by $\boldsymbol{F}$. As a benchmark quantity we study linear sampling widths, cf. (1.1) in the introduction,

$$
\varrho_{n}^{\operatorname{lin}}\left(\boldsymbol{F}, L_{q}\left(\mathbb{T}^{d}\right)\right):=\inf _{\substack{\left(\boldsymbol{\xi}_{i}\right)_{i=1}^{n} \subset \mathbb{T}^{d} \\\left(\psi_{i}\right)_{i=1}^{n} \subset L_{q}\left(\mathbb{T}^{d}\right)}} \sup _{\|f \mid \boldsymbol{F}\| \leq 1}\left\|f-\sum_{i=1}^{n} f\left(\boldsymbol{\xi}_{i}\right) \psi_{i}\right\|_{q} .
$$


This quantity can be interpreted as the minimal worst case error for the approximation of functions from the unit ball of $\boldsymbol{F}$ by linear algorithms using $n$ function evaluations and where the error is measured in $L_{q}\left(\mathbb{T}^{d}\right)$. In case of $\boldsymbol{F}=S_{p, \theta}^{\boldsymbol{r}} F\left(\mathbb{T}^{d}\right)$ with $\theta=2$ and $1<p<\infty$ we have the coincidence $S_{p}^{r} W\left(\mathbb{T}^{d}\right)=S_{p, \theta}^{r} F\left(\mathbb{T}^{d}\right)$. This case is of special interest in this section because it denotes the probably most famous representative of the $F$-scale. Choosing $m$ in (6.1) such that $n \gtrsim m^{\mu-1} 2^{m}$ an upper bound for $\varrho_{n}^{\operatorname{lin}}\left(\boldsymbol{F}, L_{q}\left(\mathbb{T}^{d}\right)\right)$ is provided by

$$
\varrho_{n}^{\operatorname{lin}}\left(\boldsymbol{F}, L_{q}\left(\mathbb{T}^{d}\right)\right) \lesssim \sup _{\|f \mid \boldsymbol{F}\| \leq 1}\left\|f-T_{m}^{L, \boldsymbol{\eta}} f\right\|_{q}
$$

Approximation with general linear information in case of mixed order Sobolev spaces $S_{p}^{r} W\left(\mathbb{T}^{d}\right)$ and Hölder-Nikolskij spaces $S_{p, \infty}^{r} B\left(\mathbb{T}^{d}\right)$ has been intensively studied in the past. We recall the concept of linear $n$-widths:

$$
\lambda_{n}\left(\boldsymbol{F}, L_{q}\left(\mathbb{T}^{d}\right)\right):=\inf _{\substack{A: \boldsymbol{F} \rightarrow L_{q}\left(\mathbb{T}^{d}\right) \\ \operatorname{rank} A \leq n}} \sup _{\|f \mid \boldsymbol{F}\| \leq 1}\|f-A(f)\|_{q} .
$$

In comparison to $\varrho_{n}^{\operatorname{lin}}\left(\boldsymbol{F}, L_{q}\left(\mathbb{T}^{d}\right)\right)$ this quantity allows to benchmark linear operators using $n$ pieces of linear information. Function evaluations are also linear information. Therefore, we have the relation

$$
\lambda_{n}\left(\boldsymbol{F}, L_{q}\left(\mathbb{T}^{d}\right)\right) \leq \varrho_{n}^{\operatorname{lin}}\left(\boldsymbol{F}, L_{q}\left(\mathbb{T}^{d}\right)\right) .
$$

That means linear $n$-widths can serve as lower bounds for linear sampling $n$-widths.

Corollary 7.1. Let $0<p<q<\infty$ and $0<\theta \leq \infty$. Additionally, the smoothness vector $\boldsymbol{r}>\frac{1}{p}$ is supposed to satisfy (1.5). Then

$$
\varrho_{n}^{\operatorname{lin}}\left(S_{p, \theta}^{r} F\left(\mathbb{T}^{d}\right), L_{q}\left(\mathbb{T}^{d}\right)\right) \lesssim\left(n^{-1} \log (\mu-1) n\right)^{r_{1}-\frac{1}{p}+\frac{1}{q}}
$$

holds for all $n>0$.

Proof. The proof follows by Theorem 6.3 with the estimate from Lemma 6.1 for the number of function evaluations used by $T_{m}^{L, \boldsymbol{\eta}}$.

Corollary 7.2. Let $\boldsymbol{r}>\frac{1}{p}$ fulfilling (1.5). Furthermore, let $1<p<q \leq 2,1 \leq \theta \leq \infty$ or $2 \leq p<q<\infty, 2 \leq \theta \leq \infty$. Then

$$
\lambda_{n}\left(S_{p, \theta}^{r} F\left(\mathbb{T}^{d}\right), L_{q}\left(\mathbb{T}^{d}\right)\right) \asymp \varrho_{n}^{l i n}\left(S_{p, \theta}^{r} F\left(\mathbb{T}^{d}\right), L_{q}\left(\mathbb{T}^{d}\right)\right) \asymp\left(n^{-1} \log (\mu-1) n\right)^{\left(r_{1}-\frac{1}{p}+\frac{1}{q}\right)}
$$

for all $n \in \mathbb{N}$.

Proof. The upper bound for $\varrho_{n}^{\text {lin }}$ follows by Corollary 7.1. The lower bounds for $\lambda_{n}$ are provided in Theorem C.2.

Remark 7.3. The result stated above is not completely new. In case $2 \leq p, \theta<q,(\theta=q)$ and $1<p, q<2$ with $\theta<q$ the upper bounds can be obtained with the help of Besov space results proven by Dinh Dũng in [10, 11] using the embedding relation $S_{p, \theta}^{r} F\left(\mathbb{T}^{d}\right) \hookrightarrow S_{p, \max \{p, \theta\}}^{r} B\left(\mathbb{T}^{d}\right)$. Nevertheless, the cases $1<p<q<2$ with $\theta>q$ and $2 \leq p<q<\theta$ are new. Compared to Besov spaces in that range of parameters we do not observe an additional logarithmic factor in the convergence rate. This parameter range includes the situation of Sobolev spaces in case $1<p<q<2$. 
The following result is based on an observation by Novak/Triebel [25] for the univariate situation.

Theorem 7.4. Let $1<p<2<q<\infty$ and $\boldsymbol{r}> \begin{cases}\frac{1}{p} & : \frac{1}{p}+\frac{1}{q} \geq 1,1 \leq \theta \leq \infty, \\ \max \left\{\frac{1}{p}, 1-\frac{1}{q}\right\} & : \frac{1}{p}+\frac{1}{q} \leq 1,1 \leq \theta \leq \infty,\end{cases}$ with (1.5). Then

$$
\lambda_{n}\left(S_{p, \theta}^{r} F\left(\mathbb{T}^{d}\right), L_{q}\left(\mathbb{T}^{d}\right)\right)=o\left(\varrho_{n}^{\operatorname{lin}}\left(S_{p, \theta}^{r} F\left(\mathbb{T}^{d}\right), L_{q}\left(\mathbb{T}^{d}\right)\right)\right)
$$

or more precisely

$$
\lambda_{n}\left(S_{p, \theta}^{r} F\left(\mathbb{T}^{d}\right), L_{q}\left(\mathbb{T}^{d}\right)\right) \lesssim n^{-\left(r_{1}-\frac{1}{p}+\frac{1}{q}\right)} \lesssim \varrho_{n}^{l i n}\left(S_{p, \theta}^{r} F\left(\mathbb{T}^{d}\right), L_{q}\left(\mathbb{T}^{d}\right)\right)
$$

holds for all $n>0$.

Proof. The bounds for $\lambda_{n}\left(S_{p, \theta}^{r} F\left(\mathbb{T}^{d}\right), L_{q}\left(\mathbb{T}^{d}\right)\right)$ come from the embedding $S_{p, \theta}^{r} F\left(\mathbb{T}^{d}\right) \hookrightarrow S_{p, \infty}^{r} B\left(\mathbb{T}^{d}\right)$ that yields

$$
\lambda_{n}\left(S_{p, \theta}^{r} F\left(\mathbb{T}^{d}\right), L_{q}\left(\mathbb{T}^{d}\right)\right) \leq \lambda_{n}\left(S_{p, \infty}^{r} B\left(\mathbb{T}^{d}\right), L_{q}\left(\mathbb{T}^{d}\right)\right) .
$$

and the results from [16, see also [12, Thm. 4.46]. The proof for the (non-sharp) lower bound of $\varrho_{n}^{\operatorname{lin}}\left(S_{p, \theta}^{r} F\left(\mathbb{T}^{d}\right), L_{q}\left(\mathbb{T}^{d}\right)\right)$ follows from the univariate situation considered in [25, Theorem 23].

Remark 7.5. The fact that the exponents of the main rate and the exponent of the logarithm in the upper bound obtained in Corollary 7.1 coincide and additionally the main rate is sharp seems to be a strong indication for the conjecture

$$
\varrho_{n}^{\operatorname{lin}}\left(S_{p, \theta}^{r} F\left(\mathbb{T}^{d}\right), L_{q}\left(\mathbb{T}^{d}\right)\right) \asymp\left(n^{-1} \log ^{\mu-1} n\right)^{r_{1}-\frac{1}{p}+\frac{1}{q}}
$$

in case $1<p<q<\infty, 1 \leq \theta \leq \infty$ and $\boldsymbol{r}>\frac{1}{p}$ with (1.5).

Sharp lower bounds for $\lambda_{n}\left(S_{p, \infty}^{r} B\left(\mathbb{T}^{d}\right), L_{q}\left(\mathbb{T}^{d}\right)\right)$ obtained in [23] yield the following observation for Hölder-Nikolskij spaces.

Corollary 7.6. Let $1<p<q \leq 2$ or $2 \leq p<q<\infty$ and $\boldsymbol{r}>\frac{1}{p}$ is supposed to satisfy (1.5). Then

$$
\varrho_{n}^{\operatorname{lin}}\left(S_{p, \infty}^{r} B\left(\mathbb{T}^{d}\right), L_{q}\left(\mathbb{T}^{d}\right)\right) \asymp \lambda_{n}\left(S_{p, \infty}^{r} B\left(\mathbb{T}^{d}\right), L_{q}\left(\mathbb{T}^{d}\right)\right) \asymp\left(n^{-1} \log (\mu-1) n\right)^{r_{1}-\frac{1}{p}+\frac{1}{q}}(\log n)^{\frac{\mu-1}{q}}
$$

holds for all $n>0$.

Proof. The upper bound was originally obtained by Dinh Dũng in [5]. The lower bound for linear widths is due to Galeev [16. In our context the upper bound for $\varrho_{n}^{\text {lin }}$ follows by Theorem 6.6 with the estimate from Lemma 6.1 for the number of function evaluations used by $T_{m}^{L, \boldsymbol{\eta}}$. The lower bound for $\lambda_{n}$ in the second case was proven recently by Malykhin and Ryutin [23], see also [16] and [12, Thm. 4.46].

Corollary 7.7. Let $1<p<2<q<\infty$ and $\boldsymbol{r}> \begin{cases}\frac{1}{p} & : \frac{1}{p}+\frac{1}{q} \geq 1, \\ \max \left\{\frac{1}{p}, 1-\frac{1}{q}\right\} & : \frac{1}{p}+\frac{1}{q}<1,\end{cases}$ fulfilling (1.5). Then

$$
\lambda_{n}\left(S_{p, \infty}^{r} B\left(\mathbb{T}^{d}\right), L_{q}\left(\mathbb{T}^{d}\right)\right)=o\left(\varrho_{n}^{\operatorname{lin}}\left(S_{p, \infty}^{r} B\left(\mathbb{T}^{d}\right), L_{q}\left(\mathbb{T}^{d}\right)\right)\right),
$$


or more precisely

$$
\lambda_{n}\left(S_{p, \infty}^{r} B\left(\mathbb{T}^{d}\right), L_{q}\left(\mathbb{T}^{d}\right)\right) \lesssim n^{-\left(r_{1}-\frac{1}{p}+\frac{1}{q}\right)} \lesssim \varrho_{n}^{l i n}\left(S_{p, \infty}^{r} B\left(\mathbb{T}^{d}\right), L_{q}\left(\mathbb{T}^{d}\right)\right)
$$

holds for all $n>0$.

Proof. The bounds for $\lambda_{n}\left(S_{p, \infty}^{\boldsymbol{r}} B\left(\mathbb{T}^{d}\right), L_{q}\left(\mathbb{T}^{d}\right)\right)$ come from [16]. The proof for the (non-sharp) lower bounds for $\varrho_{n}^{\operatorname{lin}}\left(S_{p, \infty}^{r} B\left(\mathbb{T}^{d}\right), L_{q}\left(\mathbb{T}^{d}\right)\right)$ follow from the univariate situation considered in [25, Theorem 23].

Corollary 7.8. Let $0<p, \theta<\infty(\theta=\infty)$ and the smoothness vector $\boldsymbol{r}>\frac{1}{p}$ which is supposed to satisfy (1.5) be given. Then

$$
\varrho_{n}^{\operatorname{lin}}\left(S_{p, \theta}^{\boldsymbol{r}} F\left(\mathbb{T}^{d}\right), L_{\infty}\left(\mathbb{T}^{d}\right)\right) \lesssim\left(n^{-1} \log ^{\mu-1} n\right)^{r_{1}-\frac{1}{p}} \log (\mu-1)\left(1-\frac{1}{p}\right)_{+} n
$$

holds for all $n>0$.

Proof. The upper bound follows by Theorem 6.4 with the estimate from Lemma 6.1 for the number of used function evaluations.

Based on a recent observation of Nguyen in [24, Theorem 2.15] we can state the following theorem:

Corollary 7.9. Let $1<p<2$ and $\boldsymbol{r}>1$ fulfilling (1.5). Then

$$
\lambda_{n}\left(S_{p}^{r} W\left(\mathbb{T}^{d}\right), L_{\infty}\left(\mathbb{T}^{d}\right)\right)=o\left(\varrho_{n}^{\operatorname{lin}}\left(S_{p}^{r} W\left(\mathbb{T}^{d}\right), L_{\infty}\left(\mathbb{T}^{d}\right)\right)\right)
$$

or more precisely

$$
\lambda_{n}\left(S_{p}^{r} W\left(\mathbb{T}^{d}\right), L_{\infty}\left(\mathbb{T}^{d}\right)\right) \asymp n^{-\left(r_{1}-\frac{1}{2}\right)}\left(\log ^{\mu-1} n\right)^{r_{1}} \lesssim n^{-\left(r_{1}-\frac{1}{p}\right)} \lesssim \varrho_{n}^{\operatorname{lin}}\left(S_{p}^{r} W\left(\mathbb{T}^{d}\right), L_{\infty}\left(\mathbb{T}^{d}\right)\right)
$$

holds for all $n>0$.

Proof. The bound for $\lambda_{n}\left(S_{p}^{r} W\left(\mathbb{T}^{d}\right), L_{\infty}\left(\mathbb{T}^{d}\right)\right)$ comes from Theorem C.4. The proof for the (non-sharp) lower bound for $\varrho_{n}^{\operatorname{lin}}\left(S_{p}^{r} W\left(\mathbb{T}^{d}\right), L_{\infty}\left(\mathbb{T}^{d}\right)\right)$ follows from the univariate situation considered in [25, Theorem 23].

\section{Sampling recovery and Gelfand $n$-widths}

The considerations above cover linear algorithms in the classical sense. Last but not least we consider an extension of this concept, so-called approximation using standard information, cf. [26, 27]. This means we consider algorithms that are defined as a composition of a linear information map and a possibly non-linear reconstruction operator. To avoid further technicalities we restrict to Banach spaces $\boldsymbol{F}$ that are either Sobolev spaces $S_{p}^{\boldsymbol{r}} W\left(\mathbb{T}^{d}\right)$ or Hölder-Nikolskij spaces $S_{p, \infty}^{r} B\left(\mathbb{T}^{d}\right)$ in this subsection. The non-linear sampling widths were defined in (1.2). The following relation clearly holds true

$$
\varrho_{n}\left(\boldsymbol{F}, L_{q}\left(\mathbb{T}^{d}\right) \leq \varrho_{n}^{\operatorname{lin}}\left(\boldsymbol{F}, L_{q}\left(\mathbb{T}^{d}\right)\right) .\right.
$$


Therefore (possibly non-sharp) upper bounds for sampling widths are always provided by linear sampling widths. To consider questions on optimality of these bounds we consider Gelfand $n$-widths

$$
c_{n}\left(\boldsymbol{F}, L_{q}\left(\mathbb{T}^{d}\right)\right):=\inf _{B_{\dot{B}} \boldsymbol{F} \rightarrow \mathbb{C}^{n}} \sup _{\substack{\|f \mid \boldsymbol{F}\| \leq 1 \\ \text { linear }}}\|f\|_{q} .
$$

Here $B$ denotes a general linear mapping $B: \boldsymbol{F} \rightarrow \mathbb{C}^{n}$. This means $c_{n}$ measures the minimal (over all information mappings) worst case distance of elements in the unit ball of $\boldsymbol{F}$ which can not be distinguished by the information mapping $B$. This immediately gives

$$
c_{n}\left(\boldsymbol{F}, L_{q}\left(\mathbb{T}^{d}\right)\right) \lesssim \varrho_{n}\left(\boldsymbol{F}, L_{q}\left(\mathbb{T}^{d}\right)\right) .
$$

Note that (8.1) is actually the definition of the $n$th "Gelfand numbers", which we call "Gelfand $n$-width" here. For a thorough discussion on the relation between Gelfand numbers and suitable worst-case errors we refer to the recent paper [9, Rem. 2.3]. Since Gelfand widths for embeddings id : $S_{p}^{r} W\left(\mathbb{T}^{d}\right) \rightarrow L_{q}\left(\mathbb{T}^{d}\right)$ are not studied directly we use a duality relation to Kolmogorov $n$-widths, cf. (C.1).

Lemma 8.1. The following duality relation holds true

$$
d_{n}(T: X \rightarrow Y)=c_{n}\left(T^{\prime}: Y^{\prime} \rightarrow X^{\prime}\right),
$$

where $T^{\prime}$ denotes the adjoint operator of $T$ and $X^{\prime}, Y^{\prime}$ the topological dual spaces of $X$ and $Y$.

Proof. We refer to [28, Theorem 6.2].

Corollary 8.2. Let $1<p, q<\infty$ and $\boldsymbol{r}> \begin{cases}\frac{1}{2} & : \quad 1<p<q \leq 2, \\ 1-\frac{1}{q} & : \quad p<2<q, \\ \left(\frac{1}{p}-\frac{1}{q}\right)_{+}: & \text {otherwise, }\end{cases}$

with

$$
r_{1}=\ldots=r_{\mu}<r_{\mu+1} \leq \ldots \leq r_{d}<\infty
$$

Then

$$
c_{n}\left(S_{p}^{r} W\left(\mathbb{T}^{d}\right), L_{q}\left(\mathbb{T}^{d}\right)\right) \asymp\left(n^{-1} \log ^{\mu-1} n\right)^{r_{1}-\left(\min \left\{\frac{1}{p}, \frac{1}{2}\right\}-\frac{1}{q}\right)_{+}}
$$

for all $n \in \mathbb{N}$.

Proof. The proof follows by the duality relation stated in Lemma 8.1 and a lifting argument. The topological dual spaces of $S_{p}^{r} W\left(\mathbb{T}^{d}\right)$ and $L_{q}\left(\mathbb{T}^{d}\right)$ are the spaces $S_{p^{\prime}}^{-\boldsymbol{r}} W\left(\mathbb{T}^{d}\right)$ and $L_{q^{\prime}}\left(\mathbb{T}^{d}\right)$ with $1=\frac{1}{p}+\frac{1}{p^{\prime}}=\frac{1}{q}+\frac{1}{q^{\prime}}$. Lemma 8.1 yields

$$
c_{n}\left(S_{p}^{r} W\left(\mathbb{T}^{d}\right), L_{q}\left(\mathbb{T}^{d}\right)\right)=d_{n}\left(L_{q^{\prime}}\left(\mathbb{T}^{d}\right), S_{p^{\prime}}^{-r} W\left(\mathbb{T}^{d}\right)\right) .
$$

Finally we show the identity

$$
d_{n}\left(L_{q^{\prime}}\left(\mathbb{T}^{d}\right), S_{p^{\prime}}^{-r} W\left(\mathbb{T}^{d}\right)\right) \asymp d_{n}\left(S_{q^{\prime}}^{r} W\left(\mathbb{T}^{d}\right), L_{p^{\prime}}\left(\mathbb{T}^{d}\right)\right) .
$$

For that reason we consider the lifting operator $I_{\boldsymbol{r}}$ in $D^{\prime}\left(\mathbb{T}^{d}\right)$ given by

$$
I_{\boldsymbol{r}}: f=\sum_{\boldsymbol{k} \in \mathbb{Z}^{d}} \widehat{f}(\boldsymbol{k}) e^{i \boldsymbol{k} \boldsymbol{x}} \mapsto \sum_{\boldsymbol{k} \in \mathbb{Z}^{d}} \widehat{f}(\boldsymbol{k})\left(\prod_{i=1}^{d}\left(1+\left|k_{i}\right|^{2}\right)^{-\frac{r_{i}}{2}}\right) e^{i \boldsymbol{k} \boldsymbol{x}}
$$


It is easy to check that this is an isometry that maps $f \in S_{p}^{\alpha} W$ to $I_{\boldsymbol{r}} f \in S_{p}^{\boldsymbol{\alpha}+\boldsymbol{r}} W, \alpha \in \mathbb{R}$ with $\left(I_{\boldsymbol{r}}\right)^{-1}=I_{-\boldsymbol{r}}$. Therefore we may use the commutative diagram,

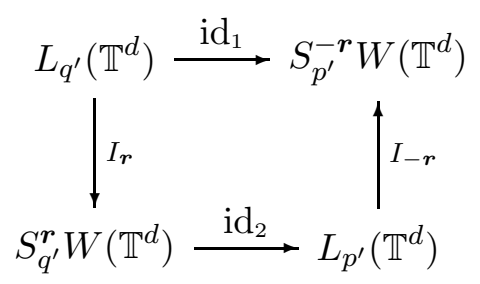

which allows to describe the operators $\mathrm{id}_{1}, \mathrm{id}_{2}$ by

$$
\operatorname{id}_{1}=I_{-\boldsymbol{r}} \circ \mathrm{id}_{2} \circ I_{\boldsymbol{r}} \quad \text { and } \quad \operatorname{id}_{2}=I_{\boldsymbol{r}} \circ \mathrm{id}_{1} \circ I_{-\boldsymbol{r}}
$$

Kolmogorov widths are $s$-numbers and fulfill a multiplicativity property that yields

$$
d_{n}\left(\mathrm{id}_{1}\right)=d_{n}\left(I_{-\boldsymbol{r}} \circ \mathrm{id}_{2} \circ I_{\boldsymbol{r}}\right) \leq\left\|I_{-\boldsymbol{r}}\right\| d_{n}\left(\mathrm{id}_{2}\right)\left\|I_{\boldsymbol{r}}\right\| \asymp d_{n}\left(\mathrm{id}_{2}\right)
$$

and

$$
d_{n}\left(\mathrm{id}_{2}\right)=d_{n}\left(L_{\boldsymbol{r}} \circ \mathrm{id}_{1} \circ L_{-\boldsymbol{r}}\right) \leq\left\|L_{\boldsymbol{r}}\right\| d_{n}\left(\mathrm{id}_{1}\right)\left\|I_{-\boldsymbol{r}}\right\| \asymp d_{n}\left(\mathrm{id}_{1}\right) .
$$

Inserting the result from Theorem C.3 finishes the proof.

Surprisingly, a new result in 23 allows us to prove the following results for Gelfand $n$ widths of Hölder spaces $S_{p, \infty}^{r} B\left(\mathbb{T}^{d}\right)$.

Theorem 8.3. Let $1<p<q<\infty$ and $\boldsymbol{r}$ with

$$
(1 / p-1 / q)_{+}<r_{1}=\ldots=r_{\mu}<r_{\mu+1} \leq \ldots \leq r_{d}<\infty
$$

then

$$
c_{n}\left(S_{p, \infty}^{r} B\left(\mathbb{T}^{d}\right), L_{q}\left(\mathbb{T}^{d}\right)\right) \asymp \begin{cases}\left(\frac{\log ^{\mu-1} n}{n}\right)^{r-\frac{1}{2}+\frac{1}{q}}(\log n)^{\frac{\mu-1}{q}} & : \quad \frac{1}{p}+\frac{1}{q}<1, p \leq 2, r_{1}>1-\frac{1}{q}, \\ \left(\frac{\log ^{\mu-1} n}{n}\right)^{r-\frac{1}{p}+\frac{1}{q}}(\log n)^{\frac{\mu-1}{q}} & : \quad 2 \leq p<q .\end{cases}
$$

Proof. The upper bounds follow from the results for linear widths in [16]. The lower bounds are new. Malykhin and Ryutin proved in 23] the following bound on Kolmogorov $n$-widths for finite dimensional normed spaces $\ell_{p}^{M}\left(\ell_{q}^{N}\right)$

$$
d_{\left\lfloor\frac{N M}{2}\right\rfloor}\left(\ell_{\infty}^{M}\left(\ell_{1}^{N}\right), \ell_{1}^{M}\left(\ell_{2}^{N}\right)\right) \asymp M .
$$

In the first case the technique for the lower bounds on linear widths presented in [16 works well also for Gelfand $n$-widths. The discretization stated there yields

$$
c_{n}\left(S_{p, \infty}^{r} B\left(\mathbb{T}^{d}\right), L_{q}\left(\mathbb{T}^{d}\right)\right) \gtrsim 2^{u\left(-r+\frac{1}{2}-\frac{1}{q}\right)} c_{n}\left(\ell_{\infty}^{u^{\mu-1}}\left(\ell_{2}^{2^{u}}\right), \ell_{q}^{u^{\mu-1} 2^{u}}\right)
$$

The duality relation in Lemma 8.1 gives

$$
c_{n}\left(S_{p, \infty}^{r} B\left(\mathbb{T}^{d}\right), L_{q}\left(\mathbb{T}^{d}\right)\right) \gtrsim 2^{u\left(-r+\frac{1}{2}-\frac{1}{q}\right)} d_{n}\left(\ell_{q^{\prime}}^{u^{\mu-1} 2^{u}}, \ell_{1}^{u^{\mu-1}}\left(\ell_{2}^{2^{u}}\right)\right) .
$$


Applying Hölders inequality in finite dimensional spaces $\ell_{p}^{M}\left(\ell_{q}^{N}\right)$ yields the following estimate

$$
c_{n}\left(S_{p, \infty}^{r} B\left(\mathbb{T}^{d}\right), L_{q}\left(\mathbb{T}^{d}\right)\right) \gtrsim 2^{u\left(-r+\frac{1}{2}-\frac{1}{q}\right)} u^{-\frac{\mu-1}{q^{\prime}}} d_{n}\left(\ell_{\infty}^{u^{\mu-1}}\left(\ell_{1}^{2^{u}}\right), \ell_{1}^{u^{\mu-1}}\left(\ell_{2}^{2^{u}}\right)\right) .
$$

Choosing $n \asymp u^{\mu-1} 2^{u}$ then the relation in (8.3) implies

$$
c_{n}\left(S_{p, \infty}^{r} B\left(\mathbb{T}^{d}\right), L_{q}\left(\mathbb{T}^{d}\right)\right) \gtrsim 2^{u\left(-r+\frac{1}{2}-\frac{1}{q}\right)} u^{\frac{\mu-1}{q}} \asymp\left(\frac{\log ^{\mu-1} n}{n}\right)^{r-\frac{1}{2}+\frac{1}{q}}(\log n)^{\frac{\mu-1}{q}} .
$$

The second case is obtained by the embedding

$$
S_{2, \infty}^{\boldsymbol{r}-\left(\frac{1}{2}-\frac{1}{p}\right)} B\left(\mathbb{T}^{d}\right) \hookrightarrow S_{p, \infty}^{\boldsymbol{r}} B\left(\mathbb{T}^{d}\right)
$$

together with the result from the first case.

Corollary 8.4. Let $2 \leq p<q<\infty$ and $\boldsymbol{r}>\frac{1}{p}$ fulfilling (1.5). Then

(i)

$$
\begin{aligned}
\varrho_{n}\left(S_{p}^{r} W\left(\mathbb{T}^{d}\right), L_{q}\left(\mathbb{T}^{d}\right)\right) & \asymp c_{n}\left(S_{p}^{r} W\left(\mathbb{T}^{d}\right), L_{q}\left(\mathbb{T}^{d}\right)\right) \asymp \varrho_{n}^{\operatorname{lin}}\left(S_{p}^{r} W\left(\mathbb{T}^{d}\right), L_{q}\left(\mathbb{T}^{d}\right)\right) \\
& \asymp \lambda_{n}\left(S_{p}^{r} W\left(\mathbb{T}^{d}\right), L_{q}\left(\mathbb{T}^{d}\right)\right) \asymp\left(n^{-1} \log ^{\mu-1} n\right)^{r_{1}-\frac{1}{p}+\frac{1}{q}},
\end{aligned}
$$

(ii)

$$
\begin{aligned}
\varrho_{n}\left(S_{p, \infty}^{r} B\left(\mathbb{T}^{d}\right), L_{q}\left(\mathbb{T}^{d}\right)\right) & \asymp c_{n}\left(S_{p, \infty}^{r} B\left(\mathbb{T}^{d}\right), L_{q}\left(\mathbb{T}^{d}\right)\right) \asymp \varrho_{n}^{l i n}\left(S_{p, \infty}^{r} B\left(\mathbb{T}^{d}\right), L_{q}\left(\mathbb{T}^{d}\right)\right) \\
& \asymp \lambda_{n}\left(S_{p, \infty}^{r} B\left(\mathbb{T}^{d}\right), L_{q}\left(\mathbb{T}^{d}\right)\right) \asymp\left(n^{-1} \log ^{\mu-1} n\right)^{r_{1}-\frac{1}{p}+\frac{1}{q}}\left(\log \frac{\mu-1}{q} n\right),
\end{aligned}
$$

holds for all $n \in \mathbb{N}$.

Proof. The proof follows by Theorems 6.3, 6.6, 8.3 and Corollary 8.2,

Remark 8.5. In the parameter range $2<p<q<\infty$ permitting non-linear reconstruction operators does not yield better results. Optimal rates can be achieved by completely linear sampling algorithms.

We obtain the following counterpart of Theorem 7.4 for non-linear sampling.

Corollary 8.6. Let $1<p<2<q<\infty$ and $\boldsymbol{r}>\max \left\{\frac{1}{p}, 1-\frac{1}{q}\right\}$ fulfilling (1.5). Additionally let $\boldsymbol{F}$ denote either $S_{p}^{\boldsymbol{r}} W\left(\mathbb{T}^{d}\right)$ or $S_{p, \infty}^{\boldsymbol{r}} B\left(\mathbb{T}^{d}\right)$. Then

$$
c_{n}\left(\boldsymbol{F}, L_{q}\left(\mathbb{T}^{d}\right)\right)=o\left(\varrho_{n}\left(\boldsymbol{F}, L_{q}\left(\mathbb{T}^{d}\right)\right)\right),
$$

or more precisely

$$
c_{n}\left(\boldsymbol{F}, L_{q}\left(\mathbb{T}^{d}\right)\right) \lesssim n^{-\left(r_{1}-\frac{1}{p}+\frac{1}{q}\right)} \lesssim \varrho_{n}\left(\boldsymbol{F}, L_{q}\left(\mathbb{T}^{d}\right)\right)
$$

holds for all $n \in \mathbb{N}$. 
Proof. The proof can be obtained by following the construction of the lower bound for the univariate situation in [25], where we recognize that the stronger inequality

$$
\varrho_{n}\left(\boldsymbol{F}, L_{q}\left(\mathbb{T}^{d}\right)\right) \geq \inf _{\left(\xi_{k}\right)_{k=1}^{n} \subset \mathbb{T}^{d}} \sup _{\substack{\|f \mid \boldsymbol{F}\| \leq 1 \\ f\left(\xi_{k}\right)=0, k=1, \ldots, n}}\|f\|_{q}
$$

holds. The estimates for $c_{n}\left(S_{p}^{r} W\left(\mathbb{T}^{d}\right), L_{q}\left(\mathbb{T}^{d}\right)\right)$ were obtained in Corollary 8.2, For $S_{p, \infty}^{r} B\left(\mathbb{T}^{d}\right)$ we refer to Theorem 8.3 .

Remark 8.7. As a consequence of the lower bound in Corollary 8.6 for $\varrho_{n}\left(\boldsymbol{F}, L_{q}\left(\mathbb{T}^{d}\right)\right)$, we obtain that in the parameter range $1<p<2<q<\infty$ even linear approximation behaves significantly better than sampling recovery with a possibly non-linear reconstructing operator.

\section{A Appendix: Tools from Fourier analysis}

The following Lemma collects trivial properties of the Fourier transform and Fourier coefficients, cf. (1.15).

Lemma A.1. Let $f \in L_{1}(\mathbb{T})$ with $\sum_{\ell \in \mathbb{Z}}|\widehat{f}(\ell)|<\infty$. Then

$$
f(\cdot)=\sum_{\ell \in \mathbb{Z}} \widehat{f}(\ell) e^{i \ell \cdot}
$$

in $C(\mathbb{T})$.

Lemma A.2 (Poisson summation). Let $f \in L_{1}(\mathbb{R})$. Then its periodization $\sum_{k \in \mathbb{Z}} f(\cdot+2 \pi k)$ converges absolutely in the norm of $L_{1}([-\pi, \pi])$. Furthermore its formal Fourier series is given by

$$
\sum_{k \in \mathbb{Z}} f(\cdot+2 \pi k)=\frac{1}{\sqrt{2 \pi}} \sum_{\ell \in \mathbb{Z}} \mathcal{F} f(\ell) e^{i \ell}
$$

Proof. We refer to [39, p. 252].

Definition A.3 (Hardy-Littlewood maximal function). Let $f \in L_{1}^{\text {loc }}\left(\mathbb{T}^{d}\right)$. Then we define the Hardy-Littlewood maximal function as

$$
M f(\boldsymbol{x}):=\sup _{Q \ni \boldsymbol{x}} \frac{1}{|Q|} \int_{Q}|f(\boldsymbol{x})| d \boldsymbol{x},
$$

where the $Q$ are cuboids centered in $\boldsymbol{x}$.

Theorem A.4 (Hardy-Littlewood maximal inequality). Let $1<p \leq \infty$ and $f \in L_{p}\left(\mathbb{T}^{d}\right)$. Then we have

$$
\left\|M f\left|L_{p}\left(\mathbb{T}^{d}\right)\|\lesssim\| f\right| L_{p}\left(\mathbb{T}^{d}\right)\right\| .
$$

For the following periodic version of the Fefferman-Stein maximal inequality we refer to [32, Prop. 3.2.4].

Theorem A.5 (Fefferman-Stein maximal inequality). Let $1<p<\infty, 1<\theta \leq \infty$ and $\left(f_{\boldsymbol{k}}\right)_{k} \subset L_{p}\left(\mathbb{T}^{d}, \ell_{\theta}\right)$. Then we have

$$
\left\|M f_{k}\left|L_{p}\left(\mathbb{T}^{d}, \ell_{\theta}\right)\|\lesssim\| f_{k}\right| L_{p}\left(\mathbb{T}^{d}, \ell_{\theta}\right)\right\| .
$$


The following pointwise bound is proved in [45, Lemma 3.3.1].

Lemma A.6. Let $a, b>0$ and

$$
f=\sum_{|k|<b} \hat{f}(k) e^{i k x}: \quad x \in \mathbb{T}
$$

be a univariate trigonometric polynomial with frequencies in $[-b, b]$. Then there exists a constant $C>0$ such that

$$
\left|\Delta_{h}^{m}(f, x)\right| \leq C \min \left\{1,|b h|^{m}\right\} \max \left\{1,|b h|^{a}\right\} P_{b, a} f(x)
$$

holds.

Lemma A.7. Let $a>0, b>0$ and $f \in C(\mathbb{T})$.

(i) If $\left|x-x_{0}\right|<\frac{1}{b}$ then

$$
\left|f\left(x_{0}\right)\right| \leq 2^{a} P_{b, a} f(x)
$$

holds.

(ii) Furthermore let $b^{\prime}>b>0$. Then

$$
P_{b, a} f(x) \leq\left(\frac{b^{\prime}}{b}\right)^{a} P_{b^{\prime}, a} f(x) .
$$

Proof. The proof is an easy exercise playing with the definition of the Peetre maximal function.

\section{B Appendix: Some multi-indexed geometric sums}

Lemma B.1. Let $\boldsymbol{r}, \boldsymbol{\eta} \in \mathbb{R}^{d}$ with $0<r_{1}=\eta_{1}=\ldots=r_{\mu}=\eta_{\mu}<r_{\mu+1} \leq \ldots \leq r_{d}$ and $r_{1}<\eta_{s}<r_{s}$ for $s=\mu+1, \ldots, d$. Then

$$
\sum_{\frac{1}{\eta_{1}} \boldsymbol{\eta} \cdot \boldsymbol{j}>m} 2^{-\boldsymbol{r} \cdot \boldsymbol{j}} \lesssim m^{\mu-1} 2^{-r_{1} m}
$$

holds for all $m \geq 1$.

Proof. We refer to [40, p. 9, Lemma B].

Lemma B.2. Let $\boldsymbol{r} \in \mathbb{R}^{d}$ with

$$
0<r_{1}=\ldots=r_{\mu}<r_{\mu+1} \leq \ldots \leq r_{d}<\infty
$$

and $\mu \leq d$. Then

$$
\sum_{\frac{1}{r_{1}} \boldsymbol{r} \cdot \boldsymbol{j} \leq m} 2^{|\boldsymbol{j}|_{1}} \asymp m^{\mu-1} 2^{m}
$$

holds for all $m \geq 1$.

Proof. We refer to [40, p. 10, Lemma D]. 


\section{Appendix: Known results on linear and Kolmogorov-widths}

Theorem C.1. Let $1<p<\infty, 1 \leq q<\infty$ and $\boldsymbol{r}>(1 / p-1 / q)_{+}$with (1.5). Then we have

$$
\lambda_{n}\left(S_{p}^{r} W\left(\mathbb{T}^{d}\right), L_{q}\left(\mathbb{T}^{d}\right)\right) \asymp \begin{cases}\left(\frac{(\log n)^{\mu-1}}{n}\right)^{r_{1}-(1 / p-1 / q)_{+}} & : \quad q \leq 2, \text { or } \quad p \geq 2, \\ \left(\frac{(\log n)^{\mu-1}}{n}\right)^{r_{1}-1 / p+1 / 2} & : \quad 1 / p+1 / q \geq 1, q>2, r>1 / p, \\ \left(\frac{(\log n)^{\mu-1}}{n}\right)^{r_{1}-1 / 2+1 / q} & : \quad 1 / p+1 / q \leq 1, p<2, r>1-1 / q .\end{cases}
$$

Proof. The case $1<q<\infty$ was proven by Galeev[15, 16], see also [13, 14]. The case $q=1$ by Romanyuk [30]. Additionally we refer to [12, Theorem 4.39] and the comments therein.

Theorem C.2. Let $\boldsymbol{r}$ as in (8.2). Let additionally $1<p<q \leq 2$ and $1 \leq \theta \leq \infty$ or $2 \leq p<q<\infty$ and $\theta \geq 2$. Then we have

$$
\lambda_{n}\left(S_{p, \theta}^{r} F\left(\mathbb{T}^{d}\right), L_{q}\left(\mathbb{T}^{d}\right)\right) \asymp\left(\frac{(\log n)^{\mu-1}}{n}\right)^{r_{1}-1 / p-1 / q},
$$

for all $n \in \mathbb{N}$.

Proof. The upper bound can be obtained for instance by sampling recovery, cf. Theorem 7.1 , We focus on lower bounds. In case $\theta \geq 2$ the embedding $S_{p}^{r} W\left(\mathbb{T}^{d}\right) \hookrightarrow S_{p, \theta}^{r} F\left(\mathbb{T}^{d}\right)$ yields

$$
\lambda_{n}\left(S_{p, \theta}^{r} F\left(\mathbb{T}^{d}\right), L_{q}\left(\mathbb{T}^{d}\right)\right) \geq \lambda_{n}\left(S_{p}^{r} W\left(\mathbb{T}^{d}\right), L_{q}\left(\mathbb{T}^{d}\right)\right)
$$

The results stated in Theorem C.1 provide the correct order. In case $\theta<p$ the embedding $S_{p, \theta}^{r} B\left(\mathbb{T}^{d}\right) \hookrightarrow S_{p, \theta}^{r} F\left(\mathbb{T}^{d}\right)$ yields

$$
\lambda_{n}\left(S_{p, \theta}^{r} F\left(\mathbb{T}^{d}\right), L_{q}\left(\mathbb{T}^{d}\right)\right) \geq \lambda_{n}\left(S_{p, \theta}^{r} B\left(\mathbb{T}^{d}\right), L_{q}\left(\mathbb{T}^{d}\right)\right) .
$$

This gives the right order in cases $1<p<q \leq 2$ and $2 \leq q<p$, cf. [29]. Finally for $\theta \geq p$ we stress on the embedding

$$
S_{p, p}^{r} B\left(\mathbb{T}^{d}\right) \hookrightarrow S_{p, \theta}^{r} F\left(\mathbb{T}^{d}\right)
$$

with

$$
\lambda_{n}\left(S_{p, \theta}^{r} F\left(\mathbb{T}^{d}\right), L_{q}\left(\mathbb{T}^{d}\right)\right) \geq \lambda_{n}\left(S_{p, p}^{r} B\left(\mathbb{T}^{d}\right), L_{q}\left(\mathbb{T}^{d}\right)\right) .
$$

This provides the lower bound in case $1<p<q \leq 2$. We refer again to [29]. by

The following is known for Kolmogorov widths in case of Sobolev spaces $S_{p}^{r} W\left(\mathbb{T}^{d}\right)$ defined

$$
d_{n}\left(S_{p}^{r} W\left(\mathbb{T}^{d}\right), L_{q}\left(\mathbb{T}^{d}\right)\right)=\inf _{\substack{A \subset L_{q}\left(\mathbb{T}^{d}\right) \\ \operatorname{dim} A \leq n}} \sup _{\left\|f \mid S_{p}^{r} W\left(\mathbb{T}^{d}\right)\right\| \leq 1} \inf _{g \in A}\|f-g\|_{q}
$$

Theorem C.3. Let $1<p, q<\infty$ and $\boldsymbol{r}> \begin{cases}\left(\frac{1}{p}-\frac{1}{q}\right)_{+} & : \quad 1 \leq p \leq q \leq 2 \text { or } 1 \leq q \leq p<\infty, \\ \max \left\{\frac{1}{2}, \frac{1}{p}\right\} & : \quad \text { otherwise, }\end{cases}$ as in (8.2). Then

$$
d_{n}\left(S_{p}^{r} W\left(\mathbb{T}^{d}\right), L_{q}\left(\mathbb{T}^{d}\right)\right) \asymp\left(m^{-1} \log ^{\mu-1} m\right)^{r_{1}-\left(\frac{1}{p}-\max \left\{\frac{1}{2}, \frac{1}{q}\right\}\right)_{+}} .
$$


Proof. The proof with every single case has a history of more than 20 years. For an overview we refer to [12, Section 4.3].

Theorem C.4. Let $1<p \leq 2$ and $\boldsymbol{r}>1$ satisfying (8.2). Then

$$
\lambda_{n}\left(S_{p}^{r} W\left(\mathbb{T}^{d}\right), L_{\infty}\left(\mathbb{T}^{d}\right)\right) \asymp n^{-\left(r_{1}-\frac{1}{2}\right)} \log ^{(\mu-1) r_{1}} n .
$$

Proof. We refer to [24, Theorem 2.14].

\section{Acknowledgment}

The authors acknowledge the fruitful discussions with D.B. Bazarkhanov, Dinh Dũng, A. Hinrichs, E. Novak and M. Ullrich on this topic. G.B and T.U. especially thank W. Sickel and V.N. Temlyakov (for asking about the Dirichlet kernel in the sampling representations) and the CRM Barcelona and S. Tikhonov for inviting them to the Intensive Research Program "Constructive Approximation and Harmonic Analysis" and the opportunity to speak about this work. G.B. and T.U. gratefully acknowledge support by the German Research Foundation (DFG) and the Emmy-Noether programme, Ul-403/1-1.

\section{References}

[1] M. Aigner and G. M. Ziegler. Proofs from The Book. Springer-Verlag, Berlin, second edition, 2001.

[2] G. Byrenheid, D. Dũng, W. Sickel, and T. Ullrich. Sampling on energy-norm based sparse grids for the optimal recovery of Sobolev type functions in $H^{\gamma}$. J. Approx. Theory, 207:207-231, 2016.

[3] G. Byrenheid and T. Ullrich. Sparse approximation with respect to the Faber-Schauder system in spaces with mixed smoothness. preprint, 2017.

[4] M. Christ and A. Seeger. Necessary conditions for vector-valued operator inequalities in harmonic analysis. Proc. London Math. Soc. (3), 93(2):447-473, 2006.

[5] D. Dũng. On optimal recovery of multivariate periodic functions. In Harmonic analysis (Sendai, 1990), ICM-90 Satell. Conf. Proc., pages 96-105. Springer, Tokyo, 1991.

[6] D. Dũng. Optimal recovery of functions of a certain mixed smoothness. Tap chí Toán Hoc J. Math., 20(2):18-32, 1992.

[7] D. Dũng. Non-linear approximations using sets of finite cardinality or finite pseudodimension. J. Complexity, 17(2):467-492, 2001. 3rd Conference of the Foundations of Computational Mathematics (Oxford, 1999).

[8] D. Dũng. B-spline quasi-interpolation sampling representation and sampling recovery in Sobolev spaces of mixed smoothness. ArXiv e-prints, Mar. 2016.

[9] S. Dirksen and T. Ullrich. Gelfand numbers related to structured sparsity and Besov space embeddings with small mixed smoothness. ArXiv e-prints arXiv:1702.06781, 2017. 
[10] D. Dũng. B-spline quasi-interpolant representations and sampling recovery of functions with mixed smoothness. J. Complexity, 27(6):541-567, 2011.

[11] D. Dũng. Sampling and cubature on sparse grids based on a B-spline quasi-interpolation. Found. Comput. Math., to appear.

[12] D. Dũng, V. Temlyakov, and T. Ullrich. Hyperbolic cross approximation. Advanced Courses in Mathematics. CRM Barcelona. Birkhuser/Springer, to appear, arXiv:1601.03978v2.

[13] D. E. Edmunds and H. Triebel. Entropy numbers and approximation numbers in function spaces. Proc. London Math. Soc. (3), 58(1):137-152, 1989.

[14] D. E. Edmunds and H. Triebel. Entropy numbers and approximation numbers in function spaces. II. Proc. London Math. Soc. (3), 64(1):153-169, 1992.

[15] È. M. Galeev. On linear widths of classes of periodic functions of several variables. Vestnik MGU, Ser.1 Mat.-Mekh., 4:13-16, 1987.

[16] È. M. Galeev. Linear widths of Hölder-Nikolskij classes of periodic functions of several variables. Mat. Zametki, 59(2):189-199, 317, 1996.

[17] M. Hansen and J. Vybíral. The Jawerth-Franke embedding of spaces with dominating mixed smoothness. Georgian Math. J., 16(4):667-682, 2009.

[18] S. Heinrich. Randomized approximation of Sobolev embeddings. II. J. Complexity, 25(5):455-472, 2009.

[19] H. Kempka. Atomic, molecular and wavelet decomposition of generalized 2-microlocal Besov spaces. J. Funct. Spaces Appl., 8(2):129-165, 2010.

[20] V. Kien Nguyen, M. Ullrich, and T. Ullrich. Change of variable in spaces of mixed smoothness and numerical integration of multivariate functions on the unit cube. ArXiv e-prints, Nov. 2015.

[21] G. Kyriazis. Decomposition systems for function spaces. Studia Math., 157(2):133-169, 2003.

[22] P. I. Lizorkin. Multipliers of Fourier integrals in the spaces $L_{p, \theta}$. Trudy Mat. Inst. Steklov, 89:231-248, 1967.

[23] Y. V. Malykhin and K. S. Ryutin. Product of octahedra is badly approximated in the $\ell_{2,1}$-metric. ArXiv e-prints, June 2016.

[24] V. K. Nguyen. Gelfand numbers of embeddings of mixed Besov spaces. preprint, 2016.

[25] E. Novak and H. Triebel. Function spaces in Lipschitz domains and optimal rates of convergence for sampling. Constructive Approximation, 23(3):325-350, 2005.

[26] E. Novak and H. Woźniakowski. Tractability of multivariate problems. Vol. 1: Linear information, volume 6 of EMS Tracts in Mathematics. European Mathematical Society (EMS), Zürich, 2008. 
[27] E. Novak and H. Woźniakowski. Tractability of multivariate problems. Volume II: Standard information for functionals, volume 12 of EMS Tracts in Mathematics. European Mathematical Society (EMS), Zürich, 2010.

[28] A. Pinkus. $N$-widths in approximation theory, volume 7 of Ergebnisse der Mathematik und ihrer Grenzgebiete (3) [Results in Mathematics and Related Areas (3)]. Springer-Verlag, Berlin, 1985.

[29] A. S. Romanyuk. Linear widths of Besov classes of periodic functions of several variables. II. Ukraïn. Mat. Zh., 53(6):820-829, 2001.

[30] A. S. Romanyuk. Best approximations and widths of classes of periodic functions of several variables. Sbornik: Mathematics, 199(2):253, 2008.

[31] H.-J. Schmeisser and W. Sickel. Sampling theory and function spaces. In Applied mathematics reviews, Vol. 1, pages 205-284. World Sci. Publ., River Edge, NJ, 2000.

[32] H.-J. Schmeisser and H. Triebel. Topics in Fourier analysis and function spaces, volume 42 of Mathematik und ihre Anwendungen in Physik und Technik [Mathematics and its Applications in Physics and Technology]. Akademische Verlagsgesellschaft Geest \& Portig K.-G., Leipzig, 1987.

[33] A. Seeger and T. Ullrich. Haar projection numbers and failure of unconditional convergence in Sobolev spaces. Math. Zeitschrift, 285(1):91-119, 2017.

[34] A. Seeger and T. Ullrich. Lower bounds for Haar projections: deterministic examples. Constr. Approx., DOI 10.1007/s00365-016-9353-3 (to appear).

[35] W. Sickel. Approximation from sparse grids and function spaces of dominating mixed smoothness. In Approximation and probability, volume 72 of Banach Center Publ., pages 271-283. Polish Acad. Sci., Warsaw, 2006.

[36] W. Sickel and T. Ullrich. The Smolyak algorithm, sampling on sparse grids and function spaces of dominating mixed smoothness. East Journal on Approximations, 13(4):387-425, 2007.

[37] W. Sickel and T. Ullrich. Spline interpolation on sparse grids. Appl. Anal., 90(3-4):337$383,2011$.

[38] S. Smolyak. Quadrature and interpolation formulas for tensor products of certain classes of functions. Soviet Mathematics, Doklady, 4:240-243, 1963.

[39] E. M. Stein and G. Weiss. Introduction to Fourier analysis on Euclidean spaces. Princeton University Press, Princeton, N.J., 1971. Princeton Mathematical Series, No. 32.

[40] V. Temlyakov. Approximation of functions with bounded mixed derivative. Proc. Steklov Inst. Math., (1(178)):vi+121, 1989. A translation of Trudy Mat. Inst. Steklov 178 (1986), Translated by H. H. McFaden.

[41] V. Temlyakov. On approximate recovery of functions with bounded mixed derivative. $J$. Complex., 9(1):41-59, Mar. 1993. 
[42] V. N. Temlyakov. Approximation of periodic functions. Computational Mathematics and Analysis Series. Nova Science Publishers, Inc., Commack, NY, 1993.

[43] H. Triebel. Theory of function spaces, volume 78 of Monographs in Mathematics. Birkhäuser Verlag, Basel, 1983.

[44] H. Triebel. Faber systems and their use in sampling, discrepancy, numerical integration. EMS Series of Lectures in Mathematics. European Mathematical Society (EMS), Zürich, 2012.

[45] T. Ullrich. Function spaces with dominating mixed smoothness, characterization by differences. Technical report, Jenaer Schriften zur Math. und Inform., Math/Inf/05/06, 2006.

[46] T. Ullrich. Smolyak's algorithm, sampling on sparse grids and Sobolev spaces of dominating mixed smoothness. East J. Approx., 14(1):1-38, 2008.

[47] J. Vybiral. Function spaces with dominating mixed smoothness. Dissertationes Math. (Rozprawy Mat.), 436:73, 2006. 